\title{
Generalized holomorphic analytic torsion
}

\author{
José Ignacio Burgos Gil Gerard Freixas i Montplet \\ Răzvan Liţcanu
}

\begin{abstract}
In this paper we extend the holomorphic analytic torsion classes of Bismut and Köhler to arbitrary projective morphisms between smooth algebraic complex varieties. To this end, we propose an axiomatic definition and give a classification of the theories of generalized holomorphic analytic torsion classes for projective morphisms. The extension of the holomorphic analytic torsion classes of Bismut and Köhler is obtained as the theory of generalized analytic torsion classes associated to $-R / 2, R$ being the $R$-genus. As application of the axiomatic characterization, we give new simpler proofs of known properties of holomorpic analytic torsion classes, we give a characterization of the $R$ genus, and we construct a direct image of hermitian structures for projective morphisms.
\end{abstract}

\section{Introduction}

The aim of this paper is to extend the classes of analytic torsion forms introduced by Bismut and Köhler to arbitrary projective morphisms between complex algebraic varieties. The main tool for this extension is an axiomatic characterization of all the possible theories of holomorphic analytic torsion classes. Before stating what we mean by a theory of holomorphic analytic torsion classes, we briefly recall the origin of the analytic torsion.

The R-torsion is a topological invariant attached to certain euclidean flat vector bundles on a finite CW-complex. This invariant was introduced by Reidemeister and generalized by Franz in order to distinguish non-homeomorphic lens spaces that have the same homology and homotopy groups. Let $W$ be a connected CW-complex and let $K$ be an orthogonal representation of $\pi_{1}(W)$. Then $K$ defines a flat vector bundle with an euclidean inner product $E_{K}$. Assume that the chain complex of $W$ with values in $E_{K}$ is acyclic. Then the R-torsion is the determinant of this complex with respect to a preferred basis.

Later, Ray and Singer introduced an analytic analogue of the R-torsion and they conjectured that, for compact riemannian manifolds, this analytic torsion agrees with the

J. I. Burgos Gil: Instituto de Ciencias Matemáticas (CSIC-UAM-UCM-UC3), Calle Nicolás Cabrera 1528049 Madrid, Spain; e-mail: burgos@icmat.es

G. Freixas i Montplet: Institut de Mathématiques de Jussieu (IMJ), Centre National de la Recherche Scientifique (CNRS), France; e-mail: freixas@math.jussieu.fr

R. Liţcanu: Faculty of Mathematics, University Al. I. Cuza Iasi, Romania; e-mail: litcanu@uaic.ro 
R-torsion. This conjecture was proved by Cheeger and Müller. If $W$ is a riemannian manifold and $K$ is as before, then we have the de Rham complex of $W$ with values in $E_{K}$ at our disposal. The hypothesis on $K$ implies that $\left(\Omega^{*}\left(W, E_{K}\right), \mathrm{d}\right)$ is also acyclic. Then the analytic torsion is essentially the determinant of the de Rham complex. Here the difficulty lies in that the vector spaces $\Omega^{p}\left(W, E_{K}\right)$ are infinite dimensional and therefore the "determinant" has to be defined using a zeta function regularization involving the laplacian. More details on the construction of R-torsion and analytic torsion can be found in [41].

Ray and Singer observed that, with the help of hermitian metrics, the acyclicity condition can be removed. Moreover, their definition of analytic torsion can be extended to any elliptic complex. In the paper [42], they introduced a holomorphic analogue of the analytic torsion as the determinant of the Dolbeault complex. They also studied some of its properties and computed some examples. In particular, they showed that this invariant depends on the complex structure and they gave a hint that the holomorphic analytic torsion should be interesting in number theory. This holomorphic analytic torsion and its generalizations are the main object of study of the present paper. Since this is the only kind of analytic torsion that we will consider, throughout the paper, by analytic torsion we will mean holomorphic analytic torsion.

In the paper [40], Quillen, using the analytic torsion, associated to each holomorphic hermitian vector bundle on a Riemann surface a hermitian metric on the determinant of its cohomology. Furthermore, he showed that this metric varies smoothly with the holomorphic structure on the vector bundle. He also computed the curvature of the hermitian line bundle on the space of all complex structures obtained in this way.

Subsequently Bismut and Freed [7], [8] generalized the construction of Quillen to families of Dirac operators on the fibers of a smooth fibration. They obtained a smooth metric and a unitary connection on the determinant bundle associated with the family of Dirac operators. Furthermore, they computed the curvature of this connection, which agrees with the degree 2 part of the differential form obtained by Bismut in his proof of the Local Index theorem [2]. Later, in a series of papers [9], [10], [11], Bismut, Gillet and Soulé considered the case of a holomorphic submersion endowed with a holomorphic hermitian vector bundle. They defined a Quillen type metric on the determinant of the cohomology of the holomorphic vector bundle. In the locally Kähler case, they showed the compatibility with the constructions of Bismut-Freed. In addition they described the variation of the Quillen metric under change of the metric on the vertical tangent bundle and on the hermitian vector bundle. The results of [9], [10], [11] represent a rigidification of [7], [8]. All in all, these works explain the relationship between analytic torsion and the Atiyah-Singer index theorem and, in the algebraic case, with Grothendieck's relative version of the Riemann-Roch theorem.

In [20], Deligne, inspired by the Arakelov formalism, gave a formula for the Quillen metric that can be seen as a very precise version of the degree one case of the RiemannRoch theorem for families of curves. This result is in the same spirit as the arithmetic Riemann-Roch theorem of Faltings [23].

In the paper [29], Gillet and Soulé conjectured an arithmetic Riemann-Roch formula that generalizes the results of Deligne and Faltings. Besides the analytic torsion or its avatar, the Quillen metric, this Riemann-Roch formula involves a mysterious new odd additive characteristic class, the $R$-genus, that they computed with the help of Zagier. 
In the work [14] Bismut and Lebeau studied the behavior of the analytic torsion with respect to complex immersions. Their compatibility formula also involved the $R$-genus. Later Bost [15] and Roessler [43] explained, using geometric arguments, why the same genus appears both in the arithmetic Riemann-Roch formula and the Bismut-Lebeau compatibility formula. However these geometric arguments do not characterize the $R$-genus.

Gillet and Soulé [30] proved the degree one part of the arithmetic Riemann-Roch theorem. A crucial ingredient of the proof is the compatibility formula of Bismut-Lebeau.

In order to establish the arithmetic Riemann-Roch theorem in all degrees it was necessary to generalize the analytic torsion and define higher analytic torsion classes. It was clear from [30] that, once a suitable theory of higher analytic torsion classes satisfying certain properties were developed, then the arithmetic Riemann-Roch theorem would follow. A first definition of such forms was given by Gillet and Soulé in [29], but they did not prove all the necessary properties. A second equivalent definition was given in [13] by Bismut and Köhler, where some of the needed properties are proved. The compatibility of higher analytic torsion classes with complex immersions, i.e. the generalization of Bismut-Lebeau compatibility formula, was proved in [3]. As a consequence, Gillet, Soulé and Rössler [25] extended the arithmetic Riemann-Roch theorem to arbitrary degrees.

In the book [24], Faltings followed a similar strategy to define direct images of hermitian vector bundles and proved an arithmetic Riemann-Roch formula up to a unique unknown odd genus.

The arithmetic Riemann-Roch theorems of Gillet-Soulé and Faltings deal only with projective morphisms between arithmetic varieties such that, at the level of complex points, define a submersion. By contrast, in his thesis [50] Zha follows a completely different strategy to establish an arithmetic Riemann-Roch theorem without analytic torsion. His formula does not involve the $R$-genus. Moreover Zha's theorem is valid for any projective morphism between arithmetic varieties.

In [44], Soulé advocates for an axiomatic characterization of the analytic torsion, similar to the axiomatic characterization of Bott-Chern classes given by Bismut-Gillet-Soulé in [9]. Note that the R-torsion has also been generalized to higher degrees giving rise to different higher torsion classes. In [33], Igusa gives an axiomatic characterization of these higher torsion classes.

We now explain more precisely what we mean by a theory of generalized analytic torsion classes. The central point is the relationship between analytic torsion and the Grothendieck-Riemann-Roch theorem.

Let $\pi: X \rightarrow Y$ be a smooth projective morphism of smooth complex varieties. Let $\omega$ be a closed $(1,1)$ form on $X$ that induces a Kähler metric on the fibers of $\pi$ and, moreover, a hermitian metric on the relative tangent bundle $T_{\pi}$. We denote $\bar{T}_{\pi}$ the relative tangent bundle provided with this metric.

Let $\bar{F}=\left(F, h^{F}\right)$ be a hermitian vector bundle on $X$ such that for every $i \geq 0, R^{i} \pi_{*} F$ is locally free. We consider on $R^{i} \pi_{*} F$ the $L^{2}$ metric obtained using Hodge theory on the fibers of $\pi$ and denote the corresponding hermitian vector bundle as $\overline{R^{i} \pi_{*} F}$. To these data, Bismut and Köhler associate an analytic torsion differential form $\tau$ that satisfies the differential equation

$$
* \partial \bar{\partial} \tau=\sum(-1)^{i} \operatorname{ch}\left(\overline{R^{i} \pi_{*} F}\right)-\pi_{*}\left(\operatorname{ch}(\bar{F}) \operatorname{Td}\left(\bar{T}_{\pi}\right)\right)
$$


where $*$ is a normalization factor that is irrelevant here (see 8). Moreover, if we consider the class of $\tau$ up to $\operatorname{Im} \partial+\operatorname{Im} \bar{\partial}$, then $\tau$ behaves nicely with respect to changes of metrics.

The Grothendieck-Riemann-Roch theorem in de Rham cohomology says that the differential form on the right side of equation (1.1) is exact. Therefore, the existence of the higher analytic torsion classes provides us an analytic proof of this theorem.

Since the Grothendieck-Riemann-Roch theorem is valid with more generality, it is natural to extend the notion of higher analytic torsion classes to non-smooth morphisms. To this end we will use the language of hermitian structures on the objects of the bounded derived category of coherent sheaves developed in [17]. In particular we will make extensive use of the category $\overline{\mathrm{D}}^{\mathrm{b}}$ introduced in loc. cit.. Since, from now on, derived categories will be the natural framework, all functors will tacitly be assumed to be derived functors. By reasons explained in loc. cit. we will restrict ourselves to the algebraic category. Let $f: X \rightarrow Y$ be a projective morphism between smooth complex algebraic varieties. Let $\bar{F}$ be a hermitian vector bundle on $X$. Now, the relative tangent complex $T_{f}$ and the derived direct image $f_{*} F$ are objects of the bounded derived category of coherent sheaves on $X$ and $Y$ respectively. Since $X$ and $Y$ are smooth, using resolutions by locally free sheaves, we can choose hermitian structures on $T_{f}$ and $f_{*} F$. Hence we have characteristic forms $\operatorname{ch}\left(\overline{f_{*} F}\right)$ and $\operatorname{Td}\left(\bar{T}_{f}\right)$. We denote by $\bar{f}$ the morphism $f$ together with the choice of hermitian structure on $T_{f}$. Then the triple $\bar{\xi}=\left(\bar{f}, \bar{F}, \overline{f_{*} F}\right)$ will be called a relative hermitian vector bundle. This is a particular case of the relative metrized complexes of Section 2 .

Then, a generalized analytic torsion class for $\bar{\xi}$ is the class modulo $\operatorname{Im} \partial+\operatorname{Im} \bar{\partial}$ of a current that satisfies the differential equation

$$
* \partial \bar{\partial} \tau=\operatorname{ch}\left(\overline{f_{*} F}\right)-f_{*}\left(\operatorname{ch}(\bar{F}) \operatorname{Td}\left(\bar{T}_{f}\right)\right) .
$$

Note that such current $\tau$ always exists. Again, the Grothendieck-Riemann-Roch theorem in de Rham cohomology implies that the right hand side of equation (1.2) is an exact current. Thus, if $Y$ is proper, the $d d^{c}$-lemma implies the existence of such a current. When $Y$ is non-proper, a compactification argument allows us to reduce to the proper case.

Of course, in each particular case, there are many choices for $\tau$. We can add to $\tau$ any closed current and obtain a new solution of equation (1.2). By a theory of generalized analytic torsion classes we mean a coherent way of choosing a solution of equation (1.2) for all relative hermitian vector bundles, satisfying certain natural minimal set of properties.

Each theory of generalized analytic torsion classes gives rise to a definition of direct images in arithmetic $K$-theory and therefore to an arithmetic Riemann-Roch formula. In fact, the arithmetic Riemann-Roch theorems of Gillet-Soulé and of Zha correspond to different choices of a theory of generalized analytic torsion classes. We leave for a subsequent paper the discussion of the relation with the arithmetic Riemann-Roch formula.

Since each projective morphism is the composition of a closed immersion followed by the projection of a projective bundle, it is natural to study first the analytic torsion classes for closed immersions and projective bundles and then combine them in a global theory of analytic torsion classes.

In [19] the authors studied the case of closed immersions (see Section 3). The generalized analytic torsion classes for closed immersions are called singular Bott-Chern classes and we will use both terms interchangeably. The definition of a theory of singular BottChern classes is obtained by imposing axioms analogous to those defining the classical 
Bott-Chern classes [26]. Namely, a theory of singular Bott-Chern classes is an assignment that, to each relative hermitian vector bundle $\bar{\xi}=\left(\bar{f}, \bar{F}, \overline{f_{*} F}\right)$, with $f$ a closed immersion, assigns the class of a current $T(\bar{\xi})$ on $Y$, satisfying the following properties:

(i) the differential equation (1.2);

(ii) functoriality for morphisms that are transverse to $f$;

(iii) a normalization condition.

A crucial observation is that, unlike the classical situation, these axioms do not uniquely characterize the singular Bott-Chern classes. Consequently there are various nonequivalent theories of singular Bott-Chern classes. They are classified by an arbitrary characteristic class of $F$ and $T_{f}$. If we further impose the condition that the theory is transitive (that is, compatible with composition of closed immersions) and compatible with the projection formula then the ambiguity is reduced to an arbitrary additive genus on $T_{f}$. The uniqueness can be obtained by adding to the conditions (i) (iii) an additional homogeneity property. The theory obtained is transitive and compatible with the projection formula and agrees (up to normalization) with the theory introduced in [12].

Similarly, one can define a theory of analytic torsion classes for projective spaces (Section 5). This is an assignment that, to each relative hermitian vector bundle $\bar{\xi}=$ $\left(\bar{f}, \bar{F}, \overline{f_{*} F}\right)$, where $f: \mathbb{P}_{Y}^{n} \rightarrow Y$ is the projection of a trivial projective bundle, assigns the class of a current $T(\bar{\xi})$ satisfying the properties analogous to (i) (iii) below, plus the additivity and the compatibility with the projection formula. The theories of analytic torsion classes for projective spaces are classified by their values in the cases $Y=$ Spec $\mathbb{C}$, $n \geq 0, F=\mathcal{O}(k), 0 \leq k \leq n$ for one particular choice of metrics (see Theorem 5.9).

We say that a theory of analytic torsion classes for closed immersions and one for projective spaces are compatible if they satisfy a compatibility equation similar to BismutLebeau compatibility formula for the diagonal immersion $\Delta: \mathbb{P}_{\mathbb{C}}^{n} \rightarrow \mathbb{P}_{\mathbb{C}}^{n} \times \mathbb{P}_{\mathbb{C}}^{n}, n \geq 0$. Given a theory of singular Bott-Chern classes that is transitive and compatible with the projection formula, there exists a unique theory of analytic torsion classes for projective spaces that is compatible with it (Theorem 6.4).

The central result of this paper (Theorem 7.7) is that, given a theory of singular BottChern classes and a compatible theory of analytic torsion classes for projective spaces, they can be combined to produce a unique theory of generalized analytic torsion classes (Definition 7.1). Moreover, every theory of analytic torsion classes arises in this way. Thus we have a complete classification of the theories of generalized analytic torsion classes by additive genera.

Once we have proved the classification theorem, we derive several applications. The first consequence of Theorem 7.7 is that the classes of the analytic torsion forms of Bismut-Köhler arise as the restriction to Kähler fibrations of the theory of generalized analytic torsion classes associated to minus one half of the $R$-genus (Theorem 8.8). In particular, we have succeeded to extend Bismut-Köhler analytic torsion classes to arbitrary projective morphisms in the algebraic category. Moreover, we reprove and generalize the theorems of Berthomieu-Bismut [1] and Ma [35], [36] on the compatibility of analytic torsion with the composition of submersions (Corollary 8.11). 
The second application of the classification theorem is a characterization of the $R$ genus. From the axiomatic point of view, the role played by the $R$-genus is mysterious. It would seem more natural to consider the generalized analytic torsion classes associated to the trivial genus 0 . This is the choice made implicitly by Zha in his thesis [50]. In fact, with our point of view, one of the main results of Zha's thesis is the existence of a theory of analytic torsion classes associated to the trivial genus. This theory leads to an arithmetic Riemann-Roch formula identical to the classical one without any correction term. Thus, one is tempted to consider the $R$-genus as an artifact of the analytic definition of the analytic torsion. Nevertheless, by the work of several authors, the $R$-genus seems to have a deeper meaning. A paradigmatic example is the computation by Bost and Kühn [34] of the arithmetic self-intersection of the line bundle of modular forms on a modular curve, provided with the Petersson metric. This formula gives an arithmetic meaning to the first term of the $R$-genus. Thus it is important to characterize the $R$-genus from an axiomatic point of view and to understand its role in the above computations.

From a theorem of Bismut [5] we know that the Bismut-Köhler analytic torsion classes of the relative de Rham complex of a Kähler fibration (with the appropriate hermitian structures) vanish. This result is important because one of the main difficulties to apply the arithmetic Riemann-Roch theorem is precisely the estimation of the analytic torsion. Moreover, this result explains why the terms of the $R$-genus appear in different arithmetic computations. For instance, the equivariant version of this result (due to Maillot and Roessler in degree 0 and to Bismut in general) allows Maillot and Roessler [37] to prove some cases of a conjecture of Gross-Deligne.

The above vanishing property characterizes the analytic torsion classes of Bismut and Köhler. In order to show this, we first construct the dual theory $T^{\vee}$ to a given theory $T$ of generalized analytic torsion classes (Theorem Definition 9.10). A theory is self-dual ( $T=$ $T^{\vee}$ ) if and only if the even coefficients of the associated genus vanish (Corollary 9.14). In particular, Bismut-Köhler's theory is self-dual. Self-duality can also be characterized in terms of the de Rham complex of smooth morphisms (Theorem 9.18). A theory $T$ is selfdual if its components of bidegree $(2 p-1, p), p$ odd, in the Deligne complex, vanish on the relative de Rham complexes of Kähler fibrations. Finally, in Theorem 9.24 we show that, if it exists, a theory of analytic torsion classes that vanishes, on all degrees, on the relative de Rham complexes of Kähler fibrations is unique, hence it agrees with BismutKöhler's one. In fact, to characterize this theory, it is enough to assume the vanishing of the analytic torsion classes for the relative de Rham complexes of Kähler fibrations of relative dimension one. To establish this characterization we appeal to the non-vanishing of the tautological class $\kappa_{g-2}$ on the moduli stack $\mathcal{M}_{g}$ of smooth curves of genus $g \geq 2$.

The third application of generalized analytic torsion classes is the construction of direct images of hermitian structures. We consider the category $\overline{\mathbf{S m}}_{* / \mathbb{C}}$ introduced in [17]. The objects of this category are smooth complex varieties, and the morphisms are projective morphisms equipped with a hermitian structure on the relative tangent complex. Assume that we have chosen a theory of generalized analytic torsion classes. Let $\bar{f}: X \rightarrow Y$ be a morphism in $\overline{\mathbf{S m}}_{* / \mathbb{C}}$. One would like to define a direct image functor $f_{*}: \overline{\mathbf{D}}^{\mathrm{b}}(X) \rightarrow \overline{\mathbf{D}}^{\mathrm{b}}(Y)$. It turns out that, using analytic torsion, we can not define the direct image functor on the category $\overline{\mathbf{D}}^{\mathrm{b}}$ and we have to introduce a new category $\widehat{\mathbf{D}}^{\mathrm{b}}$. 
Roughly speaking, the relation between $\widehat{D}^{\mathrm{b}}$ and $\mathrm{D}^{\mathrm{b}}$, is the same as the relation between the arithmetic $K$-groups and the usual $K$-groups ([28]). Then we are able to define a direct image functor $f_{*}: \widehat{\mathbf{D}}^{\mathrm{b}}(X) \rightarrow \widehat{\mathbf{D}}^{\mathrm{b}}(Y)$, that satisfies the composition rule, projection formula and base change. Moreover, if the theory of generalized analytic torsion is self-dual (as the Bismut-Köhler theory) this functor satisfies a Grothendieck duality theorem. In a forthcoming paper, the direct image functor will be the base of an arithmetic Grothendieck-Riemann-Roch theorem for projective morphisms.

The last application that we discuss is a new proof of a theorem of Bismut-Bost on the singularity of the Quillen metric for degenerating families of curves, whose singular fibers have at most ordinary double points [6]. In contrast with loc. cit., where the spectral definition of the Ray-Singer analytic torsion is required, our arguments rely on the existence of a generalized theory for arbitrary projective morphisms and some elementary computations of Bott-Chern classes. This theorem has already been generalized by Bismut [4] and Yoshikawa [49] to families of varieties of arbitrary dimension. In fact, our approach is very similar to the one in [4] and [49]. One of the main ingredients of their proof is Bismut-Lebeau immersion formula, while our approach uses implicitly Bismut's generalization of the immersion formula in the comparison between Bismut-Köhler analytic torsion and a theory of generalized analytic torsion classes. But what we want to emphasize is that, once we have identified Bismut-Köhler as (part of) a theory of generalized analytic torsion classes, many arguments can be simplified considerably because the theory has been extended to non-smooth projective morphism. For simplicity, we treat only the case of families of curves and the Quillen metric, but the methods can be applied to higher dimensional families and analytic torsion forms of higher degree.

A few words about notations. The normalizations of characteristic classes and BottChern classes in this paper differ from the ones used by Bismut, Gillet-Soule and other authors. The first difference is that they work with real valued characteristic classes, while we use characteristic classes in Deligne cohomology, that naturally include the algebrogeometric twist. The second difference is a factor $1 / 2$ in Bott-Chern classes, that explains the factor $1 / 2$ that appears in the characteristic class associated to the torsion classes of Bismut-Köhler. This change of normalization appears already in [16] and its objective is to avoid the factor $1 / 2$ that appears in the definition of arithmetic degree in [27, §3.4.3] and the factor 2 that appears in [27, Theorem 3.5.4] when relating Green currents with Beilinson regulator. The origin of this factor is that the natural second order differential equation that appears when defining Deligne-Beilinson cohomology is $\mathrm{d}_{\mathcal{D}}=-2 \partial \bar{\partial}$, while the operator used when dealing with real valued forms is $\mathrm{d} \mathrm{d}^{c}=\frac{1}{4 \pi i} \mathrm{~d}_{\mathcal{D}}$. Thus the characteristic classes that appear in the present article only agree with the ones in the papers of Bismut, Gillet and Soule after renormalization. With respect to the work of these authors we have also changed the sign of the differential equation that characterizes singular Bott-Chern classes. In this way, the same differential equation appears when considering both, singular Bott-Chern classes and analytic torsion classes. This change is necessary to combine them.

We point out that our construction of generalized analytic torsion classes is influenced by the thesis of Zha [50], where the author uses implicitly a theory of analytic torsion classes different from that of Bismut-Köhler.

In the unpublished e-print [46], L. Weng gives another axiomatic approach to analytic 
torsion classes, only for smooth morphisms between Kähler fibrations. This forces him to include a continuity condition with respect to the deformation to the normal cone as one of the axioms. The remaining axioms he uses are: the differential equation, functoriality with respect to cartesian squares, compatibility with respect to the projection formula and two anomaly formulas. A collection of differential forms satisfying these axioms are called relative Bott-Chern secondary characteristic classes. These characteristic classes are not unique. The main result of Weng's paper is that any two such theories are related by an additive genus. Moreover he is able to obtain a weak form of the existence theorem for relative Bott-Chern secondary characteristic classes.

Further applications of the generalized analytic torsion classes are left for future work. We plan to prove generalizations of the arithmetic Grothendieck-Riemann-Roch theorem of Gillet-Soulé [30] and Gillet-Rössler-Soulé [25] to arbitrary projective morhisms, along the lines of [19].

It is possible to compute explicitly the characteristic numbers of the unique theory of analytic torsion classes for projective spaces compatible with the homogeneous theory for closed immersions. This computation makes the characterization of generalized analytic torsion classes more precise. Nevertheless, since this computation is much more transparent when written in terms of properties of arithmetic Chow groups and the Riemann-Roch theorem, we leave it to the paper devoted to the arithmetic Riemann-Roch theorem.

We also plan to study the possible axiomatic characterization of equivariant analytic torsion classes. Note that the characterization of equivariant singular Bott-Chern forms has already been obtained by Tang in [45].

\section{Deligne complexes, transverse morphisms and relative metrized complexes}

In this section we fix the notations and conventions used through the article, we also recall the definition of transverse morphisms and we review some basic properties. Finally we introduce the notion of relative metrized complex, and explain some basic constructions.

The natural context where one can define the Bott-Chern classes and the analytic torsion classes is that of Deligne complexes. For the convenience of the reader we will summarize in this section the basic facts about the Deligne complexes we will use in the sequel. For more details the reader is referred to [16] and [18].

Definition 2.1. A Dolbeault complex $A=\left(A_{\mathbb{R}}^{*}, \mathrm{~d}_{A}\right)$ is a bounded below graded complex of real vector spaces equipped with a compatible bigrading on $A_{\mathbb{C}}=A_{\mathbb{R}} \otimes_{\mathbb{R}} \mathbb{C}$, i.e.,

$$
A_{\mathbb{C}}^{n}=\bigoplus_{p+q=n} A^{p, q}
$$

satisfying the following properties:

(i) The differential $d_{A}$ can be decomposed as the sum $d_{A}=\partial+\bar{\partial}$ of operators $\partial$ of type $(1,0)$, respectively $\bar{\partial}$ of type $(0,1)$.

(ii) The symmetry property $\overline{A^{p, q}}=A^{q, p}$ holds, where - denotes complex conjugation. 
The basic example of Dolbeault complex is the complex of differential forms on a smooth variety $X$ over $\mathbb{C}$, denoted $E^{*}(X)_{\mathbb{R}}$.

Following [18, §5.2], to a Dolbeault complex one assigns a Deligne complex denoted $\mathcal{D}^{*}(A, *)$. In this paper we will only use the following pieces of this complex:

$$
\begin{aligned}
\mathcal{D}^{2 p+1}(A, p) & =\left(A^{p, p+1} \oplus A^{p+1, p}\right) \cap(2 \pi i)^{p} A_{\mathbb{R}}^{2 p+1}, \\
\mathcal{D}^{2 p}(A, p) & =A^{p, p} \cap(2 \pi i)^{p} A_{\mathbb{R}}^{2 p}, \\
\mathcal{D}^{2 p-1}(A, p) & =A^{p-1, p-1} \cap(2 \pi i)^{p-1} A_{\mathbb{R}}^{2 p-2}, \\
\mathcal{D}^{2 p-2}(A, p) & =\left(A^{p-2, p-1} \oplus A^{p-1, p-2}\right) \cap(2 \pi i)^{p-1} A_{\mathbb{R}}^{2 p-3} .
\end{aligned}
$$

The differential of the Deligne complex, denoted by $\mathrm{d}_{\mathcal{D}}: \mathcal{D}^{n}(A, p) \rightarrow \mathcal{D}^{n+1}(A, p)$ is given, in the above degrees by

$$
\begin{aligned}
\text { if } \eta & \in \mathcal{D}^{2 p}(A, p), & \mathrm{d}_{\mathcal{D}} \eta & =\mathrm{d} \eta, \\
\text { if } \eta & \in \mathcal{D}^{2 p-1}(A, p), & \mathrm{d}_{\mathcal{D}} \eta & =-2 \partial \bar{\partial} \eta, \\
\text { if } \eta=(u, v) & \in \mathcal{D}^{2 p-2}(A, p), & \mathrm{d}_{\mathcal{D}} \eta & =-\partial u-\bar{\partial} v .
\end{aligned}
$$

When $A$ is a Dolbeault algebra, that is, $A$ is a graded commutative real differential algebra and the product is compatible with the bigrading, then $\mathcal{D}^{*}(A, *)$ has a product

$$
\bullet: \mathcal{D}^{n}(A, p) \otimes \mathcal{D}^{m}(A, q) \longrightarrow \mathcal{D}^{n+m}(A, p+q)
$$

that is graded commutative with respect to the first degree, it is associative up to homotopy and satisfies the Leibnitz rule. The only case where we will need the explicit formula for the product is for $\omega \in \mathcal{D}^{2 p}(A, p)$ and $\eta \in \mathcal{D}^{m}(A, q): \omega \bullet \eta=\omega \wedge \eta$.

The Deligne algebra of differential forms on $X$ is defined to be

$$
\mathcal{D}^{*}(X, *):=\mathcal{D}^{*}\left(E^{*}(X)_{\mathbb{R}}, *\right) .
$$

If $X$ is equi-dimensional of dimension $d$, there is a natural trace map given by

$$
\int: H_{c}^{2 d}(X, \mathbb{R}(d)) \rightarrow \mathbb{R}, \quad \omega \longmapsto \frac{1}{(2 \pi i)^{d}} \int_{X} \omega
$$

To take this trace map into account the Dolbeault complex of currents is constructed as follows. Denote by $E_{c}^{*}(X)_{\mathbb{R}}$ the space of differential forms with compact support. Then $D_{p, q}(X)$ is the topological dual of $E_{c}^{p, q}(X)$ and $D_{n}(X)_{\mathbb{R}}$ is the topological dual of $E_{c}^{n}(X)_{\mathbb{R}}$. In this complex the differential is given by

$$
\mathrm{d} T(\eta)=(-1)^{n} T(\mathrm{~d} \eta)
$$

for $T \in D_{n}(X)_{\mathbb{R}}$. For $X$ equi-dimensional of dimension $d$ we write

$$
D^{p, q}(X)=D_{d-p, d-q}(X), \quad D^{n}(X)_{\mathbb{R}}=(2 \pi i)^{-d} D_{2 d-n}(X) .
$$

With these definitions, $D^{*}(X)_{\mathbb{R}}$ is a Dolbeault complex and it is a Dolbeault module over $E^{*}(X)_{\mathbb{R}}$. We will denote

$$
\mathcal{D}_{D}^{*}(X, *):=\mathcal{D}^{*}\left(D^{*}(X)_{\mathbb{R}}, *\right) .
$$


for the Deligne complex of currents on $X$. The trace map above defines an element

$$
\delta_{X} \in \mathcal{D}_{D}^{0}(X, 0)
$$

More generally, if $Y \subset X$ is a subvariety of pure codimension $p$, then the current integration along $Y$, denoted $\delta_{Y} \in \mathcal{D}_{D}^{2 p}(X, p)$ is given by

$$
\delta_{Y}(\omega)=\frac{1}{(2 \pi i)^{d-p}} \int_{Y} \omega
$$

Moreover, if $S \subset T^{*} X_{0}$ is a closed conical subset of the cotangent bundle of $X$ with the zero section removed, we will denote by $\left(\mathcal{D}_{D}^{*}(X, S, *), \mathrm{d}_{\mathcal{D}}\right)$ the Deligne complex of currents on $X$ whose wave front set is contained in $S$. For instance, if $N_{Y}^{*}$ is the conormal bundle to $Y$, then

$$
\delta_{Y} \in \mathcal{D}_{D}^{2 p}\left(X, N_{Y}^{*}, p\right)
$$

If $\omega$ is a locally integrable differential form, we associate to it a current

$$
[\omega](\eta)=\frac{1}{(2 \pi i)^{\operatorname{dim} X}} \int_{X} \eta \wedge \omega .
$$

This map induces an isomorphism $\mathcal{D}^{*}(X, *) \rightarrow \mathcal{D}_{D}^{*}(X, \emptyset, *)$ that we use to identify them. For instance, when in a formula sums of currents and differential forms appear, we will tacitly assume that the differential forms are converted into currents by this map.

Note also that, if $f: X \rightarrow Y$ is a proper morphism of smooth complex varieties of relative dimension $e$, then there are direct image morphisms

$$
f_{*}: \mathcal{D}_{D}^{n}(X, p) \longrightarrow \mathcal{D}_{D}^{n-2 e}(X, p-e) .
$$

If $f$ is smooth, the direct image of differential forms is defined by, first converting them into currents and then applying the above direct image of currents. If $f$ is a smooth morphism of relative dimension $e$ we can convert them back into differential forms. This procedure gives us $1 /(2 \pi i)^{e}$ times the usual integration along the fiber.

We shall use the notations and definitions of [19]. In particular, we write

$$
\begin{aligned}
& \widetilde{\mathcal{D}}^{n}(X, p)=\mathcal{D}^{n}(X, p) / \mathrm{d}_{\mathcal{D}} \mathcal{D}^{n-1}(X, p), \\
& \widetilde{\mathcal{D}}_{D}^{n}(X, p)=\mathcal{D}_{D}^{n}(X, p) / \mathrm{d}_{\mathcal{D}} \mathcal{D}_{D}^{n-1}(X, p) .
\end{aligned}
$$

We now recall the definition of the set of normal direction of a map and the definition of transverse morphisms.

Definition 2.2. Let $f: X \rightarrow Y$ be a morphism of smooth complex varieties. Let $T^{*} Y_{0}$ be the cotangent bundle to $Y$ with the zero section removed. The set of normal directions of $f$ is the conic subset of $T^{*} Y_{0}$ given by

$$
N_{f}=\left\{(y, v) \in T^{*} Y_{0} \mid \mathrm{d} f^{t} v=0\right\} .
$$

Definition 2.3. Let $f: X \rightarrow Y$ and $g: Z \rightarrow Y$ be morphisms of smooth complex varieties. We say that $f$ and $g$ are transverse if $N_{f} \cap N_{g}=\emptyset$. 
It is easily seen that, if $f$ is a closed immersion, this definition of transverse morphisms agrees with that given in [31, IV-17.13]. If $f$ and $g$ are transverse, then the cartesian product $X \underset{Y}{\times} Z$ is smooth. For lack of a good reference we prove the following result.

Proposition 2.4. Let $f: X \rightarrow Y$ and $g: Z \rightarrow Y$ be transverse morphisms of smooth complex varieties. Then they are tor-independent.

Proof. Since the conditions of being transverse and being tor-independent are both local on $Y, X$ and $Z$ we may assume that the map $f$ factorizes as $X \stackrel{i}{\rightarrow} Y \times \mathbb{A}^{n} \stackrel{p}{\rightarrow} Y$, where $i$ is a closed immersion and $p$ is the projection. Let $g^{\prime}: Z \times \mathbb{A}^{n} \rightarrow Y \times \mathbb{A}^{n}$ be the morphism $g \times$ id. If $f$ and $g$ are transverse then $i$ and $g^{\prime}$ are transverse. While, if $i$ and $g^{\prime}$ are torindependent then $f$ and $g$ are tor-independent. Hence we may suppose that $f$ is a closed immersion.

Since every closed immersion between smooth schemes is regular, we may assume that $Y=\operatorname{Spec} A, X=\operatorname{Spec} A / I$, where $I$ is an ideal generated by a regular sequence $\left(s_{1}, \ldots, s_{k}\right)$ and $Z=\operatorname{Spec} B$. The transversality condition implies that $\left(s_{1}, \ldots, s_{k}\right)$ is a regular sequence generating $I B$. Let $K$ be the Koszul resolution of $A / I$ attached to the above sequence. Then $K \otimes_{A} B$ is the Koszul resolution of $B / I B$, hence exact. Therefore, $\operatorname{Tor}_{A}^{i}(A / I, B)=0$ for all $i \geq 1$. Thus $f$ and $g$ are tor-independent.

Let now $Y^{\prime \prime} \stackrel{h}{\rightarrow} Y^{\prime} \stackrel{g}{\rightarrow} Y$ be morphisms of smooth complex varieties such that $g$ and $g \circ h$ are smooth. We form the cartesian diagram

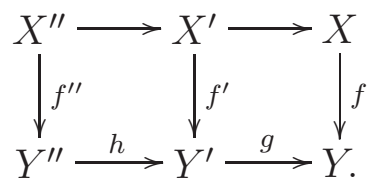

The smoothness of $g$ implies that $N_{f^{\prime}}=g^{*} N_{f}$. Then the smoothness of $g \circ h$ implies that $h$ and $f^{\prime}$ are transverse. Therefore, any current $\eta \in \mathcal{D}_{D}^{*}\left(Y^{\prime}, N_{f^{\prime}}, *\right)$ can be pulled back to a current $h^{*} \eta \in \mathcal{D}_{D}^{*}\left(Y^{\prime \prime}, N_{f^{\prime \prime}}, *\right)$.

The following result will be used to characterize several Bott-Chern classes and analytic torsion classes.

Lemma 2.5. Let $f: X \rightarrow Y$ be a morphism of smooth complex varieties. Let $\widetilde{\varphi}$ be an assignment that, to each smooth morphism of complex varieties $g: Y^{\prime} \rightarrow Y$ and each acyclic complex $\bar{A}$ of hermitian vector bundles on $X^{\prime}:=\underset{Y}{\times} Y^{\prime}$ assigns a class

$$
\widetilde{\varphi}(\bar{A}) \in \bigoplus_{n, p} \widetilde{\mathcal{D}}_{D}^{n}\left(Y^{\prime}, g^{*} N_{f}, p\right)
$$

fulfilling the following properties:

(i) (Differential equation) the equality $\mathrm{d}_{\mathcal{D}} \widetilde{\varphi}(\bar{A})=0$ holds;

(ii) (Functoriality) for each morphism $h: Y^{\prime \prime} \rightarrow Y^{\prime}$ of smooth complex varieties with $g \circ h$ smooth, the relation $h^{*} \widetilde{\varphi}(\bar{A})=\widetilde{\varphi}\left(h^{*} \bar{A}\right)$ holds; 
(iii) (Normalization) if $\bar{A}$ is orthogonally split, then $\widetilde{\varphi}(\bar{A})=0$.

Then $\widetilde{\varphi}=0$.

Proof. The argument of the proof of [19. Thm. 2.3] applies mutatis mutandis to the present situation. One only needs to observe that all the operations with differential forms of that argument can be extended to the currents that appear in the present situation thanks to the hypothesis about their wave front sets.

In the paper [17] we defined and studied hermitian structures on objects of the bounded derived category of coherent sheaves on a smooth complex variety. The language and the results of loc. cit. will be used extensively in this paper. We just mention here that a hermitian metric on an object $\mathcal{F}$ of $\mathbf{D}^{\mathrm{b}}(X)$ is an isomorphism $E \rightarrow \mathcal{F}$ in $\mathbf{D}^{\mathrm{b}}(X)$, with $E$ a bounded complex of vector bundles, together with a choice of a hermitian metric on each constituent vector bundle of $E$. Such an isomorphism always exists due to the fact that we work in the algebraic category. A hermitian structure is an equivalence class of hermitian metrics. To each smooth complex variety $X$, we associated the category $\overline{\mathbf{D}}^{\mathrm{b}}(X)([17, \S 3])$ whose objects are objects of $\mathrm{D}^{\mathrm{b}}(X)$ provided with a hermitian structure. We introduced the hermitian cone ([17, Def. 3.14]), denoted cone, of a morphism in $\overline{\mathbf{D}}^{\mathrm{b}}(X)$. We also defined Bott-Chern classes for isomorphisms ([17, Thm. 4.11]) and distinguished triangles ([17, Thm. 4.18]) in $\overline{\mathrm{D}}^{\mathrm{b}}(X)$. We introduced a universal abelian group for additive BottChern classes. Namely, the set of hermitian structures on a zero object of $\mathbf{D}^{\mathrm{b}}(X)$ is an abelian group that we denote $\mathbf{K A}(X)$ ([17, Def. 2.31]). Finally, we defined the category $\overline{\mathbf{S m}}_{* / \mathbb{C}}([17, \S 5])$ whose objects are smooth complex varieties and whose morphisms are projective morphisms together with a hermitian structure on the relative tangent complex.

We introduce now one of the central objects of the paper.

Definition 2.6. Let $f: X \rightarrow Y$ be a projective morphism of smooth complex varieties and $\bar{f} \in \operatorname{Hom}_{\overline{\operatorname{Sm}}_{* / \mathbb{C}}}(X, Y)$ a morphism over $f$. Let $\overline{\mathcal{F}} \in \mathrm{Ob}_{\overline{\mathbf{D}}}^{\mathrm{b}}(X)$ and let $\overline{f_{*} \mathcal{F}} \in$ $\operatorname{Ob} \overline{\mathbf{D}}^{\mathrm{b}}(Y)$ be an object over $f_{*} \mathcal{F}$. The triple $\bar{\xi}=\left(\bar{f}, \overline{\mathcal{F}}, \overline{f_{*} \mathcal{F}}\right)$ will be called a relative metrized complex. When $f$ is a closed immersion we will also call it an embedded metrized complex. When $\overline{\mathcal{F}}$ and $\overline{f_{*} \mathcal{F}}$ are clear from the context we will denote the relative metrized complex $\bar{\xi}$ by the morphism $\bar{f}$.

Let $\bar{\xi}=\left(\bar{f}, \overline{\mathcal{F}}, \overline{f_{*} \mathcal{F}}\right)$ be a relative metrized complex and let $g: Y^{\prime} \rightarrow Y$ be a morphism of smooth complex varieties that is transverse to $f$. Consider the cartesian diagram

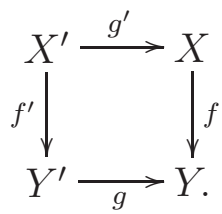

Then $f^{\prime}$ is still projective. Moreover, the transversality condition implies that the canonical morphism $g^{\prime *} T_{\bar{f}} \rightarrow-\rightarrow T_{f^{\prime}}$ is a hermitian structure on $T_{f^{\prime}}$. We define

$$
g^{*} \bar{f}=\left(f^{\prime}, g^{\prime *} T_{\bar{f}}\right) \in \operatorname{Hom}_{\overline{\mathbf{S m}}_{* / \mathbb{C}}}\left(X^{\prime}, Y^{\prime}\right) .
$$


By tor-independence, there is a canonical isomorphism $g^{*} f_{*} \mathcal{F} \rightarrow f_{*}^{\prime} g^{\prime *} \mathcal{F}$. Therefore $g^{*} \overline{f_{*} \mathcal{F}}$ induces a hermitian structure on $f_{*}^{\prime} g^{\prime *} \mathcal{F}$.

Definition 2.9. The pull-back of $\bar{\xi}$ by $g$ is the relative metrized complex

$$
g^{*} \bar{\xi}=\left(g^{*} \bar{f}, g^{\prime *} \overline{\mathcal{F}}, g^{*} \overline{f_{*} \mathcal{F}}\right) .
$$

Definition 2.10. Let $\bar{\xi}=\left(\bar{f}: X \rightarrow Y, \overline{\mathcal{F}}, \overline{f_{*} \mathcal{F}}\right)$ be a relative metrized complex. Let $\overline{\mathcal{G}}$ be an object of $\overline{\mathbf{D}}^{\mathrm{b}}(Y)$. The hermitian structures on $\overline{f_{*} \mathcal{F}}$ and $\overline{\mathcal{G}}$ induce a natural hermitian structure on $f_{*}\left(\mathcal{F} \otimes f^{*} \mathcal{G}\right)$ that we denote $\overline{f_{*} \mathcal{F}} \otimes \overline{\mathcal{G}}$. The tensor product of $\bar{\xi}$ by $\overline{\mathcal{G}}$ is then defined to be the relative metrized complex

$$
\bar{\xi} \otimes \overline{\mathcal{G}}=\left(\bar{f}, \overline{\mathcal{F}} \otimes f^{*} \overline{\mathcal{G}}, \overline{f_{*} \mathcal{F}} \otimes \overline{\mathcal{G}}\right) .
$$

Definition 2.11. Let $\bar{\xi}_{i}=\left(\bar{f}, \overline{\mathcal{F}_{i}}, \overline{f_{*} \mathcal{F}_{i}}\right), i=1,2$ be relative metrized coherent complexes on $X$. Then the direct sum relative metrized complex is

$$
\bar{\xi}_{1} \oplus \bar{\xi}_{2}:=\left(\bar{f}, \overline{\mathcal{F}_{1}} \oplus \overline{\mathcal{F}_{2}}, \overline{f_{*} \mathcal{F}_{1}} \oplus \overline{f_{*} \mathcal{F}_{2}}\right) .
$$

We now introduce a notation for Todd-twisted direct images of currents and differential forms, that will simplify many formulas involving the Todd genus. Let $\bar{f}=\left(f, \bar{T}_{f}\right)$ be a morphism in $\overline{\mathbf{S m}}_{* / \mathbb{C}}$. To $\bar{f}$ we associate a Todd differential form $\operatorname{Td}(\bar{f}):=\operatorname{Td}\left(\bar{T}_{f}\right) \in$ $\bigoplus_{p} \mathcal{D}^{2 p}(X, p)$ [17, (5.15)]. Let $S$ be a closed conic subset of $T^{*} X_{0}$. Then we denote

$$
f_{*}(S)=\left\{(f(x), \eta) \in T^{*} Y_{0} \mid\left(x,(\mathrm{~d} f)^{t} \eta\right) \in S\right\} \cup N_{f} .
$$

If $g: Y \rightarrow Z$ is another morphism of smooth complex varieties, it is easy to see that we have $(g \circ f)_{*}(S) \subseteq g_{*} f_{*}(S)$.

Definition 2.13. Let $\bar{f}: X \rightarrow Y$ be a morphism in $\overline{\mathbf{S m}}_{* / \mathbb{C}}$ of relative dimension $e$. For each closed conical subset $S \subset T^{*} X_{0}$ and each pair of integers $n$, $p$, we define the map

$$
\bar{f}_{\mathrm{b}}: \mathcal{D}_{D}^{n}(X, S, p) \rightarrow \mathcal{D}_{D}^{n-2 e}\left(Y, f_{*} S, p-e\right), \quad \bar{f}_{\mathrm{b}}(\omega)=f_{*}(\omega \bullet \operatorname{Td}(\bar{f})) .
$$

Proposition 2.14. Let $\bar{f}: X \rightarrow Y$ and $\bar{g}: Y \rightarrow Z$ be morphisms in $\overline{\mathbf{S m}}_{* / \mathbb{C}}$ of relative dimensions $e_{1}$ and $e_{2}$ respectively. Let $S \subset T^{*} X_{0}, T \subset T^{*} Y_{0}$ be closed conical subsets and let $\bar{h}=\bar{f} \circ \bar{g}$, of relative dimension $e=e_{1}+e_{2}$.

(i) The following diagram is commutative

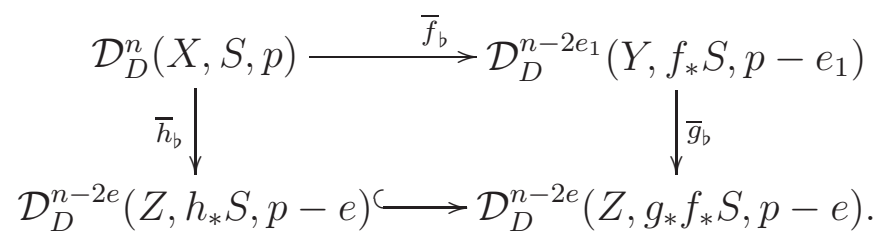

(ii) Let $\theta \in \mathcal{D}_{D}^{m}(X, S, q)$ and $\omega \in \mathcal{D}_{D}^{n}(Y, T, p)$. Assume $T \cap N_{f}=\emptyset$ and that $T+f_{*} S$ is disjoint with the zero section in $T^{*} Y_{0}$. Then $f^{*} T+S$ is disjoint with the zero section and there is an equality of currents

$$
\bar{f}_{\mathrm{b}}\left(f^{*}(\omega) \bullet \theta\right)=\omega \bullet \bar{f}_{\mathrm{b}}(\theta)
$$

in $\mathcal{D}_{D}^{n+m}(Y, W, p+q)$, with $W=f_{*}\left(S+f^{*} T\right) \cup f_{*} S \cup f_{*} f^{*} T$. 
Proof. For the first assertion, it is enough to notice the equality of currents

$$
\left.\bar{g}_{b}\left(\bar{f}_{b}(\omega)\right)=(g \circ f)_{*}\left(\omega \bullet f^{*} \operatorname{Td}(\bar{g}) \bullet \operatorname{Td}(\bar{f})\right)\right) .
$$

For the second, it is easy to see that $f^{*} T+S$ does not cross the zero section, and hence both sides of the equality are defined. It then suffices to establish the equality of currents $f_{*}\left(f^{*} \omega \bullet \theta\right)=f_{*}(\omega) \bullet \theta$. If $\theta$ and $\omega$ are smooth, then the equality follows from the definitions. The general case follows by approximation of $\theta$ and $\omega$ by smooth currents and the continuity of the operators $f^{*}$ and $f_{*}$.

Proposition 2.15. Let $\bar{f}$ be a morphism in $\overline{\mathbf{S m}}_{* / \mathbb{C}}$ of relative dimension e and $S$ a closed conical subset of $T^{*} X_{0}$. Let $g: Y^{\prime} \rightarrow Y$ be a morphism of smooth complex varieties transverse to $f$. Consider the cartesian diagram (2.7) and let $\bar{f}^{\prime}=g^{*} \bar{f}$. Suppose that $N_{g^{\prime}}$ is disjoint with S. Then:

(i) $N_{g}$ and $f_{*} S$ are disjoint and $g^{*} f_{*} S \subset f_{*}^{\prime} g^{\prime *} S$;

(ii) the following diagram commutes:

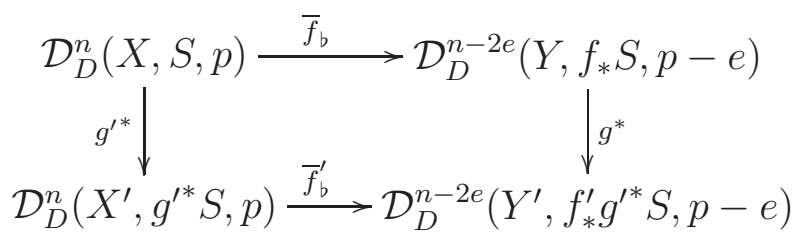

Proof. The first claim follows from the definitions. In particular the diagram makes sense. For the commutativity of the diagram, we observe that, since $g^{\prime *} \operatorname{Td}(\bar{f})=\operatorname{Td}\left(\bar{f}^{\prime}\right)$, it suffices to check the equality of currents $g^{*} f_{*}(\theta)=f^{\prime}{ }_{*} g^{\prime *}(\theta)$ for $\theta \in \mathcal{D}_{D}^{n}(X, S, p)$.

By the continuity of the operators $g^{*}, g^{\prime *}, f_{*}$ and $f^{\prime}{ }_{*}$, it is enough to prove the relation whenever $\theta$ is smooth. Moreover, using a partition of unity argument we are reduced to the following local analytic statement.

Lemma 2.16. Let $f: X \rightarrow Y$ and $g: Y^{\prime} \rightarrow Y$ be transverse morphisms of complex manifolds. Let $\theta$ be a smooth differential form on $X$ with compact support. Consider the diagram (2.7). Then

$$
g^{*} f_{*}(\theta)={f^{\prime}}_{*} g^{\prime *}(\theta) .
$$

Proof. The map $f$ can be factored as $X \stackrel{\varphi}{\longrightarrow} X \times Y \stackrel{p_{2}}{\longrightarrow} Y$, where $\varphi(x)=(x, f(x))$ is a closed immersion and $p_{2}$, the second projection, is smooth. Using again the continuity of the operators $g^{*}$ (respectively $g^{\prime *}$ ) and $f_{*}$ (respectively $f^{\prime *}$ ), we are reduced to prove the equation (2.17) in the case when $f$ is smooth and in the case when $f$ is a closed immersion. The case when $f$ is smooth is clear. Assume now that $f$ is a closed immersion. By transversality, $f^{\prime}$ is also a closed immersion of complex manifolds. We may assume that $\theta=f^{*} \widetilde{\theta}$ for some smooth form $\tilde{\theta}$ on $Y$. Then equation (2.17) follows from the chain of equalities

$$
g^{*} f_{*} \theta=g^{*} f_{*} f^{*} \widetilde{\theta}=g^{*}\left(\widetilde{\theta} \wedge \delta_{X}\right)=g^{*}(\widetilde{\theta}) \wedge \delta_{X^{\prime}}=f_{*}^{\prime} f^{*} g^{*} \widetilde{\theta}=f_{*}^{\prime} g^{\prime *} f^{*} \widetilde{\theta}=f_{*}^{\prime} g^{\prime *} \theta .
$$

This concludes the proof of the lemma and the proposition. 


\section{Analytic torsion for closed immersions}

In the paper [19] the authors study the singular Bott-Chern classes associated to closed immersions of smooth complex varieties. The singular Bott-Chern classes are the analogue, for closed immersions, of the analytic torsion for smooth morphisms. For this reason, we will call them also analytic torsion classes. The aim of this section is to recall the main results of [19] and to translate them into the language of derived categories.

Definition 3.1. A theory of analytic torsion classes for closed immersions is a map that, to each embedded metrized complex $\bar{\xi}=\left(\bar{f}: X \rightarrow Y, \overline{\mathcal{F}}, \overline{f_{*} \mathcal{F}}\right)$ assigns a class

$$
T(\bar{\xi}) \in \bigoplus_{p} \widetilde{\mathcal{D}}_{D}^{2 p-1}\left(Y, N_{f}, p\right)
$$

satisfying the following conditions.

(i) (Differential equation) The equality $\mathrm{d}_{\mathcal{D}} T(\bar{\xi})=\operatorname{ch}\left(\overline{f_{*} \mathcal{F}}\right)-\bar{f}_{\mathrm{b}}[\operatorname{ch}(\overline{\mathcal{F}})]$ holds.

(ii) (Functoriality) For every morphism $h: Y^{\prime} \rightarrow Y$ of smooth complex varieties that is transverse to $f$ we have the equality $h^{*} T(\bar{\xi})=T\left(h^{*} \bar{\xi}\right)$.

(iii) (Normalization) If $X=\emptyset$ (hence $\overline{\mathcal{F}}=\overline{0}$ ), $Y=\operatorname{Spec} \mathbb{C}$, and $\overline{f_{*} \mathcal{F}}=\overline{0}$, then $T(\bar{f}, \overline{0}, \overline{0})=0$.

Definition 3.2. Let $T$ be a theory of analytic torsion classes for closed immersions.

(i) We say that $T$ is compatible with the projection formula if, for every embedded metrized complex $\bar{\xi}=\left(\bar{f}, \overline{\mathcal{F}}, \overline{f_{*} \mathcal{F}}\right)$, and every object $\overline{\mathcal{G}} \in \overline{\mathbf{D}}^{\mathrm{b}}(Y)$, we have

$$
T(\bar{\xi} \otimes \overline{\mathcal{G}})=T(\bar{\xi}) \bullet \operatorname{ch}(\overline{\mathcal{G}}) .
$$

(ii) We say that $T$ is additive if, given $\bar{\xi}_{i}=\left(\bar{f}, \overline{\mathcal{F}_{i}}, \overline{f_{*} \mathcal{F}_{i}}\right), i=1,2$, two embedded metrized complexes, we have

$$
T\left(\bar{\xi}_{1} \oplus \bar{\xi}_{2}\right)=T\left(\bar{\xi}_{1}\right)+T\left(\bar{\xi}_{2}\right) .
$$

(iii) We say that $T$ is transitive if, given a embedded metrized complex $\bar{\xi}=\left(\bar{f}, \overline{\mathcal{F}}, \overline{f_{*} \mathcal{F}}\right)$, a closed immersion of smooth complex varieties $g: Y \rightarrow Z$, a morphism $\bar{g}$ over $g$, and an object $\overline{(g \circ f)_{*} \mathcal{F}} \in \operatorname{Ob} \overline{\mathbf{D}}^{\mathrm{b}}(Z)$ over $(g \circ f)_{*} \mathcal{F}$, we have

$$
T(\bar{g} \circ \bar{f})=T(\bar{g})+\bar{g}_{b}(T(\bar{f})) .
$$

Remark 3.6. (i) If $T$ is well defined for objects of $\overline{\mathbf{D}}^{\mathrm{b}}$, then the normalization condition in Definition 3.1 and the normalization condition in [19, Def. 6.9] are equivalent. The compatibility with the projection formula implies the normalization condition and the additivity (see [19, Prop. 10.9])

(ii) To check that a theory is compatible with the projection formula, it is enough to consider complexes consisting of a single hermitian vector bundle in degree 0 . 
Let $X$ be a smooth complex variety and let $\bar{N}$ be a hermitian vector bundle of rank $r$. We denote by $P=\mathbb{P}(N \oplus \mathbf{1})$ the projective bundle obtained by completing $N$. Let $\pi_{P}: P \rightarrow X$ be the projection and let $s: X \rightarrow P$ be the zero section. Since $N$ can be identified with the normal bundle to $X$ on $P$, the hermitian metric of $\bar{N}$ induces a hermitian structure on $s$. We will denote it by $\bar{s}$. On $P$ we have a tautological quotient vector bundle with an induced metric $\bar{Q}$. For each hermitian vector bundle $\bar{F}$ on $X$ we have the Koszul resolution $K(F, N)$ of $s_{*} F$. We denote by $K(\bar{F}, \bar{N})$ the Koszul resolution with the induced metrics. See [19, Def. 5.3] for details.

Definition 3.7. Let $T$ be a theory of analytic torsion classes for closed immersions. We say that $T$ is homogeneous if, for every pair of hermitian vector bundles $\bar{N}$ and $\bar{F}$ with $\operatorname{rk} N=r$, there exists a homogeneous class of bidegree $(2 r-1, r)$ in the Deligne complex

$$
\widetilde{e}(\bar{F}, \bar{N}) \in \widetilde{\mathcal{D}}_{D}^{2 r-1}\left(P, N_{s}, r\right)
$$

such that

$$
T(\bar{s}, \bar{F}, K(\bar{F}, \bar{N})) \bullet \operatorname{Td}(\bar{Q})=\widetilde{e}(\bar{F}, \bar{N}) \bullet \operatorname{ch}\left(\pi_{P}^{*} \bar{F}\right)
$$

Remark 3.9. Observe that Definition 3.7 is equivalent to [19, Def. 9.2]. The advantage of the definition in this paper is that it treats on equal footing the case when $\mathrm{rk} F=0$.

Let $\mathbb{D}$ denote the base ring for Deligne cohomology (see [19] before Definition 1.5). A consequence of [19, Thm. 1.8] is that there is a bijection between the set of additive genus in Deligne cohomology and the set of power series in one variable $\mathbb{D}[[x]]$. To each power series $\varphi \in \mathbb{D}[[x]]$ it corresponds the unique additive genus such that $\varphi(L)=\varphi\left(c_{1}(L)\right)$ for every line bundle $L$.

Definition 3.10. A real additive genus is an additive genus such that the corresponding power series belongs to $\mathbb{R}[[x]]$.

Let $1_{1} \in \mathbb{D}$ be the element represented by the constant function 1 of $\mathcal{D}^{1}(\operatorname{Spec} \mathbb{C}, 1)=$ $\mathbb{R}$. The main result of [19] can be written as follows.

Theorem 3.11. (i) There is a unique homogeneous theory of analytic torsion classes for closed immersions, that we denote $T^{h}$. This theory is compatible with the projection formula, additive and transitive.

(ii) Let $T$ be any transitive theory of analytic torsion classes for closed immersions, that is compatible with the projection formula. Then there is a unique real additive genus $S_{T}$ such that, for any embedded metrized complex $\bar{\xi}:=\left(\bar{f}, \overline{\mathcal{F}}, \overline{f_{*} \mathcal{F}}\right)$, we have

$$
T(\bar{\xi})-T^{h}(\bar{\xi})=-f_{*}\left[\operatorname{ch}(\mathcal{F}) \bullet \operatorname{Td}\left(T_{f}\right) \bullet S_{T}\left(T_{f}\right) \bullet \mathbf{1}_{1}\right]
$$

(iii) Conversely, any real additive genus $S$ defines, by means of equation (3.12), a unique transitive theory of analytic torsion classes $T_{S}$ for closed immersions, that is compatible with the projection formula and additive. 
Proof. Existence and uniqueness for both $T^{h}$ and $T_{S}$ is the content of [19] when restricting to triples $\bar{\xi}$ with $T_{\bar{f}}=\bar{N}_{X / Y}[-1], \overline{\mathcal{F}}$ a hermitian vector bundle placed in degree 0 and $\overline{f_{*} \mathcal{F}}$ given by a finite locally free resolution. For the general case, we thus need to prove that the theories of analytic torsion classes for closed immersions in the sense of loc. cit. uniquely extend to arbitrary $\bar{\xi}$, fulfilling the desired properties.

Assume given a theory $T$ in the sense of [19], compatible with the projection formula and transitive. We will call $T$ the initial theory. We consider a triple $\bar{\xi}$ with $T_{\bar{f}}=$ $\bar{N}_{X / Y}[-1]$ and $\overline{\mathcal{F}} \in \mathrm{Ob} \overline{\mathbf{D}}^{\mathrm{b}}(X)$. Choose a representative $\bar{F} \rightarrow \mathcal{F}$ of the hermitian structure on $\overline{\mathcal{F}}$. We then define $T(\bar{\xi})$ by induction on the length of the complex $F$. First suppose that $F=F^{d}[-d]$ consists of a single vector bundle placed in degree $d$. Choose a finite locally free resolution

$$
\cdots \rightarrow E^{1} \rightarrow E^{0} \rightarrow f_{*} F^{d} \rightarrow 0 .
$$

Endow the vector bundles $E^{i}$ with smooth hermitian metrics. Observe that there is an induced isomorphism $\bar{E}[-d] \stackrel{\sim}{\sim} \overline{f_{*} \mathcal{F}}$, in $\overline{\mathbf{D}}^{\mathrm{b}}(Y)$, whose Bott-Chern classes ch are defined in [17, $\S 4]$. We then put

$$
T(\bar{\xi})=(-1)^{d} T\left(\bar{N}_{X / Y}, \bar{F}^{d}, \bar{E}\right)+\widetilde{\operatorname{ch}}\left(\bar{E}[-d] \stackrel{\sim}{\sim-\rightarrow} \overline{f_{*} \mathcal{F}}\right) .
$$

This definition does not depend on the choice of representative of the hermitian structure on $\overline{\mathcal{F}}$, nor on the choice of $\bar{E}$, due to [17, Thm. 4.11, Prop. 4.13], and [19, Cor. 6.14]. The differential equation is satisfied as a consequence of the differential equations for $T\left(\bar{N}_{X / Y}, \bar{F}^{d}, \bar{E}\right)$ and $\widetilde{c h}\left(\bar{E}[-d] \stackrel{\sim}{\sim} \overline{f_{*} \mathcal{F}}\right)$. The compatibility with pull-back by morphisms $h: Y^{\prime} \rightarrow Y$ transverse to $f$ is immediate as well. Finally, notice that by construction, if $\bar{\xi}^{\prime}=\left(\bar{N}_{X / Y}, \overline{\mathcal{F}}, \bar{f}_{*} \overline{\mathcal{F}}^{\prime}\right)$, then

$$
T\left(\overline{\xi^{\prime}}\right)=T(\bar{\xi})+\widetilde{\operatorname{ch}}\left({\overline{f_{*}}}^{\prime}, \overline{f_{*} \mathcal{F}}\right) .
$$

Now suppose that $T(\bar{\xi})$ has been defined for $F$ of length $l$, satisfying in addition (3.14). If $F$ has length $l+1$, let $F^{d}$ be the first non-zero vector bundle of $F$. Consider the exact sequence of complexes

$$
0 \rightarrow \sigma^{>d} \bar{F} \rightarrow \bar{F} \rightarrow \bar{F}^{d}[-d] \rightarrow 0
$$

where $\sigma^{>d}$ is the bête filtration. Observe that as a distinguished triangle, $(\bar{\varepsilon})$ is tightly distinguished, hence $\widetilde{c h}(\bar{\varepsilon})=0$. Choose hermitian metrics on $f_{*} \sigma^{>d} F$ and $f_{*} F^{d}[-d]$. We thus have a distinguished triangle in $\overline{\mathbf{D}}^{\mathrm{b}}(Y)$

$$
\overline{f_{*} \sigma^{>d} F} \rightarrow \overline{f_{*} F} \rightarrow \overline{f_{*} F^{d}}[-d] \rightarrow \overline{f_{*} \sigma^{>d} F}[1] \rightarrow \ldots
$$

We define

$$
T(\bar{\xi})=T\left(\bar{N}_{X / Y}, \sigma^{>d} \bar{F}, \overline{f_{*} \sigma^{>d} F}\right)+(-1)^{d} T\left(\bar{N}_{X / Y}, \bar{F}^{d}, \bar{f}_{*} F^{d}\right)-\widetilde{c h}(\bar{\tau}) .
$$

This does not depend on the choice of hermitian structures on $f_{*} \sigma^{>d} F$ and $f_{*} F^{d}$, by the analogue to [17, Thm. 3.33 (vii)] for $\widetilde{c h}$ and because (3.14) holds by assumption 
for $T\left(\bar{N}_{X / Y}, \sigma^{>d} \bar{F}, \overline{f_{*} \sigma^{>d} F}\right)$ and $T\left(\bar{N}_{X / Y}, \bar{F}^{d}, \bar{f}_{*} F^{d}\right)$. Similarly, (3.14) holds for $T(\bar{\xi})$ defined in (3.15). The differential equation and compatibility with pull-back are proven as in the first case. This concludes the proof of the existence in case that $T_{\bar{f}}=\bar{N}_{X / Y}[-1]$.

To conclude with the existence, we may now consider a general $\bar{\xi}$. Choose a hermitian metric on the normal bundle $N_{X / Y}$. Put $\bar{\xi}^{\prime}=\left(\bar{N}_{X / Y}[-1], \overline{\mathcal{F}}, \overline{f_{*} \mathcal{F}}\right)$. We define

$$
T(\bar{\xi})=T\left(\bar{\xi}^{\prime}\right)+\bar{f}_{b}\left[\operatorname{ch}(\overline{\mathcal{F}}) \bullet \widetilde{T d}_{m}\left(T_{\bar{f}}-\rightarrow \bar{N}_{X / Y}[-1]\right)\right],
$$

where $\widetilde{\mathrm{Td}}_{m}$ is the multiplicative Todd secondary class defined in [17, $\left.\S 5\right]$. It is straightforward from the definition that $T(\bar{\xi})$ satisfies the differential equation and is compatible with pull-back by morphisms transverse to $f$. We call $T$ the extended theory.

We now proceed to prove that the extended theory $T$ is transitive and compatible with the projection formula. For the projection formula, it suffices by Remark 3.6(ii) to prove

$$
T(\bar{\xi} \otimes \bar{G})=T(\bar{\xi}) \bullet \operatorname{ch}(\bar{G})
$$

for $\bar{G}$ a hermitian vector bundle placed in degree 0 . This readily follows from the inductive construction of the extended theory $T$ and the assumptions on the initial theory $T$. One similarly establishes the transitivity and the additivity

We conclude by observing that, since Lemma 2.5 implies that the equations (3.13), (3.14), (3.15) and (3.16) hold, the theory $T(\bar{\xi})$ thus constructed for arbitrary $\bar{\xi}$ is completely determined by the values $T\left(\bar{\xi}^{\prime}\right)$, with $\bar{\xi}^{\prime}$ of the form $\left(\bar{N}_{X / Y}, \bar{F}, \bar{E}\right)$ where $\bar{F}$ is a hermitian vector bundle and $E \rightarrow f_{*} F$ is a finite locally free resolution.

Once we have seen that any theory of singular Bott-Chern classes as in [19] can be uniquely extended, then statements (ii) and (iii) follow combining equation (7.3) and Corollary 9.43 in [19]. Note that the minus sign in equation (3.12) comes from the fact that $S\left(T_{f}\right)=-S\left(N_{X / Y}\right)$.

In [19, §6] several anomaly formulas are proved. We now indicate the translation of these formulas to the current setting. Recall that we are using the notation of [17] with respect to secondary characteristic classes.

Proposition 3.17. Let $T$ be a theory of analytic torsion classes for closed immersions. Let $\bar{\xi}=\left(\bar{f}: X \rightarrow Y, \overline{\mathcal{F}}, \overline{f_{*} \mathcal{F}}\right)$ be an embedded metrized complex.

(i) If $\overline{\mathcal{F}}^{\prime}$ is another choice of metric on $\mathcal{F}$ and $\bar{\xi}_{1}=\left(\bar{f}: X \rightarrow Y, \overline{\mathcal{F}}^{\prime}, \overline{f_{*} \mathcal{F}}\right)$, then

$$
T\left(\bar{\xi}_{1}\right)=T(\bar{\xi})+\bar{f}_{b}\left[\widetilde{c h}\left(\overline{\mathcal{F}}^{\prime}, \overline{\mathcal{F}}\right)\right]
$$

(ii) If $\bar{f}^{\prime}$ is another hermitian structure on $f$ and $\bar{\xi}_{2}=\left(\bar{f}^{\prime}: X \rightarrow Y, \overline{\mathcal{F}}, \overline{f_{*} \mathcal{F}}\right)$, then

$$
T\left(\bar{\xi}_{2}\right)=T(\bar{\xi})+\bar{f}_{b}^{\prime}\left[\operatorname{ch}(\overline{\mathcal{F}}) \bullet \widetilde{T d}_{m}\left(\bar{f}^{\prime}, \bar{f}\right)\right]
$$

(iii) If ${\bar{f} \mathcal{F}^{\prime}}^{\prime}$ is another choice of metric on $f_{*} \mathcal{F}$, and $\bar{\xi}_{3}=\left(\bar{f}: X \rightarrow Y, \overline{\mathcal{F}}, \bar{f}_{*} \overline{\mathcal{F}}^{\prime}\right)$, then

$$
T\left(\bar{\xi}_{3}\right)=T(\bar{\xi})-\widetilde{\operatorname{ch}}\left({\overline{f_{*} \mathcal{F}}}^{\prime}, \overline{f_{*} \mathcal{F}}\right) .
$$


Proof. We first prove the second assertion. Let $\bar{E} \rightarrow T_{f}$ be a representative of the hermitian structure on $T_{\bar{f}}$. By [17, Thm. 3.13 (ii)], we may assume the hermitian structure on $T_{\bar{f}^{\prime}}$ is represented by the composition

$$
\bar{E} \oplus \bar{A} \stackrel{\mathrm{pr}_{1}}{\longrightarrow} \bar{E} \rightarrow T_{f}
$$

for some bounded acyclic complex $\bar{A}$ of hermitian vector bundles on $X$. For every smooth morphism $g: Y^{\prime} \rightarrow Y$ of complex varieties, consider the cartesian diagram (2.7). We introduce the assignment that, to every such $g$ and each bounded acyclic complex of hermitian vector bundles $\bar{A}$ on $X^{\prime}$, assigns the class

$$
\begin{aligned}
\widetilde{\varphi}(\bar{A})= & T\left(g^{\prime * \bar{\xi}}\right)-T\left(\left(f^{\prime}, g^{\prime *} T_{\bar{f}}+[\bar{A}]\right), g^{\prime *} \overline{\mathcal{F}}, g^{*} \overline{f_{*} \mathcal{F}}\right) \\
& +f^{\prime}{ }_{*}\left[\operatorname{ch}\left(g^{*} \overline{\mathcal{F}}\right) \widetilde{T d}_{m}\left(\left(g^{\prime *} T_{\bar{f}}+[\bar{A}]\right), g^{\prime *} T_{\bar{f}}\right) \operatorname{Td}\left(g^{\prime *} T_{\bar{f}}+[\bar{A}]\right)\right] .
\end{aligned}
$$

Here $[\bar{A}]$ stands for the class of $\bar{A}$ in $\mathbf{K A}\left(X^{\prime}\right)$ ([17, Def. 2.31]) and + denotes the action of $\mathbf{K A}\left(X^{\prime}\right)$ on $\overline{\mathbf{D}}^{\mathrm{b}}\left(X^{\prime}\right)$ ([17, Thm. 3.13]). Since $\widetilde{\varphi}$ satisfies the hypothesis of Lemma 2.5, we have $\widetilde{\varphi}=0$. This concludes the proof of (ii).

To prove (i), we observe that $\overline{\mathcal{F}}^{\prime}=\overline{\mathcal{F}}+[\bar{A}]$ for some bounded acyclic complex $\bar{A}$ of hermitian vector bundles on $X$. For each cartesian diagram as (2.7), we set $\bar{f}^{\prime}=g^{*} \bar{f}$. Let $\widetilde{\varphi}_{1}$ be the assignment that, to each such diagram and each bounded acyclic complex of hermitian vector bundles $\bar{A}$ on $X^{\prime}$, assigns the class

$$
\widetilde{\varphi}_{1}(\bar{A})=T\left(g^{\prime * \bar{\xi}}\right)-T\left(\bar{f}^{\prime}, g^{\prime *} \overline{\mathcal{F}}+[A], g^{*} \overline{f_{*} \mathcal{F}}\right)-\bar{f}_{b}^{\prime}[\widetilde{c h}(A)] .
$$

The hypothesis of Lemma 2.5 are satisfied, hence $\widetilde{\varphi}_{1}=0$. This concludes the proof of (i)

Finally, to prove (iii), to each morphism $g: Y^{\prime} \rightarrow Y$, transverse to $f$, we associate the cartesian diagram (2.7) and we consider the assignment $\widetilde{\varphi}_{2}$ that, to each bounded acyclic complex of hermitian vector bundles $\bar{B}$ on $Y^{\prime}$, assigns the class

$$
\widetilde{\varphi}_{2}(\bar{B})=T\left(g^{\prime * \bar{\xi}}\right)-T\left(\bar{f}^{\prime}, g^{\prime *} \overline{\mathcal{F}}, g^{*} \overline{f_{*} \mathcal{F}}+[B]\right)+\widetilde{c h}(B) .
$$

By Lemma 2.5 applied to $\operatorname{id}_{Y}$, we have $\widetilde{\varphi}_{2}=0$. This concludes the proof of (iii)

The following result provides a compatibility relation for analytic torsion classes for closed immersions with respect to distinguished triangles. The statement is valid for additive theories, in particular the ones we are concerned with.

Proposition 3.19. Let $T$ be an additive theory of analytic torsion classes for closed immersions. Let $f: X \rightarrow Y$ be a closed immersion of smooth complex varieties. Consider distinguished triangles in $\overline{\mathrm{D}}^{\mathrm{b}}(X)$ and $\overline{\mathrm{D}}^{\mathrm{b}}(Y)$ respectively,

$$
(\bar{\tau}): \overline{\mathcal{F}}_{2} \rightarrow \overline{\mathcal{F}}_{1} \rightarrow \overline{\mathcal{F}}_{0} \rightarrow \overline{\mathcal{F}}_{2}[1], \quad\left(\overline{f_{*} \tau}\right): \bar{f}_{*} \mathcal{F}_{2} \rightarrow{\overline{f_{*} \mathcal{F}_{1}}}_{f_{*} \overline{\mathcal{F}}_{0}} \rightarrow{\overline{f_{*}}}_{2}[1],
$$

and the relative hermitian complexes $\bar{\xi}_{i}=\left(\bar{f}, \overline{\mathcal{F}}_{i}, \bar{f}_{*} \mathcal{F}_{i}\right), i=0,1,2$. Then we have:

$$
\sum_{j}(-1)^{j} T\left(\bar{\xi}_{j}\right)=\widetilde{\operatorname{ch}}\left(\overline{f_{*} \tau}\right)-\bar{f}_{b}(\widetilde{\operatorname{ch}}(\bar{\tau}))
$$


Proof. We can assume that the distinguished triangles $\bar{\tau}$ and $\overline{f_{*} \tau}$ can be represented by short exact sequences of complexes of hermitian vector bundles

$$
\begin{array}{cl}
\bar{\varepsilon}: & 0 \longrightarrow \bar{E}_{2} \longrightarrow \bar{E}_{1} \longrightarrow \bar{E}_{0} \longrightarrow 0, \\
\bar{\nu}: & 0 \longrightarrow \bar{V}_{2} \longrightarrow \bar{V}_{1} \longrightarrow \bar{V}_{0} \longrightarrow 0 .
\end{array}
$$

Applying the explicit construction at the beginning of the proof of [19, Theorem 2.3] to each row of the above exact sequences, we obtain exact sequences

$$
\begin{aligned}
& \widetilde{\varepsilon}^{i}: 0 \longrightarrow \widetilde{E}_{2}^{i} \longrightarrow \widetilde{E}_{1}^{i} \longrightarrow \widetilde{E}_{0}^{i} \longrightarrow 0, \\
& \widetilde{\nu}^{i}: 0 \longrightarrow \widetilde{V}_{2}^{i} \longrightarrow \widetilde{V}_{1}^{i} \longrightarrow \widetilde{V}_{0}^{i} \longrightarrow 0
\end{aligned}
$$

over $X \times \mathbb{P}^{1}$ and $Y \times \mathbb{P}^{1}$ respectively. The restriction of $\widetilde{\varepsilon}^{i}$ (respectively $\widetilde{\nu}^{i}$ ) to $X \times\{0\}$ (respectively $Y \times\{0\}$ ) agrees with $\bar{\varepsilon}$ (respectively $\bar{\nu}$ ). Whereas the restriction to $X \times$ $\{\infty\}$ (respectively $Y \times\{\infty\}$ ) is orthogonally split. The sequences $\widetilde{\varepsilon}^{i}$ and $\widetilde{\nu}^{i}$ form exact sequences of complexes that we denote $\widetilde{\varepsilon}$ and $\widetilde{\nu}$. It is easy to verify that the restriction to $X \times\{\infty\}$ (respectively $Y \times\{\infty\}$ ) are orthogonally split as sequences of complexes. Moreover, there are isomorphisms $\widetilde{V}_{j} \rightarrow f_{*} \widetilde{E}_{j}, j=0,1,2$. We denote $\widetilde{\xi}_{j}=(\bar{f} \times$ $\left.\operatorname{id}_{\mathbb{P}^{1}}, \widetilde{E}_{j}, \widetilde{V}_{j}\right)$. Then, in the group $\bigoplus_{p} \widetilde{\mathcal{D}}_{D}^{2 p-1}\left(Y, N_{f}, p\right)$, we have

$$
\begin{aligned}
0 & =\mathrm{d}_{\mathcal{D}} \frac{1}{2 \pi i} \int_{\mathbb{P}^{1}} \frac{-1}{2} \log t \bar{t} \bullet \sum_{j}(-1)^{j} T\left(\widetilde{\xi}_{j}\right) \\
& =T\left(\bar{\xi}_{1}\right)-T\left(\bar{\xi}_{0} \oplus \bar{\xi}_{2}\right)-\frac{1}{2 \pi i} \int_{\mathbb{P}^{1}} \frac{-1}{2} \log t \bar{t} \bullet \sum_{j}(-1)^{j} \operatorname{ch}\left(\widetilde{V}_{j}\right) \\
& +\frac{1}{2 \pi i} \int_{\mathbb{P}^{1}} \frac{-1}{2} \log t \bar{t} \bullet \sum_{j}(-1)^{j}\left(f \times \operatorname{id}_{\mathbb{P}^{1}}\right)_{*}\left(\operatorname{ch}\left(\widetilde{E}_{j}\right) \bullet \operatorname{Td}\left(\bar{f} \times \operatorname{id}_{\mathbb{P}^{1}}\right)\right) \\
& =T\left(\bar{\xi}_{1}\right)-T\left(\bar{\xi}_{0} \oplus \bar{\xi}_{2}\right)+\widetilde{\operatorname{ch}}\left(\overline{f_{*} \tau}\right)-f_{*}(\widetilde{\operatorname{ch}}(\bar{\tau}) \operatorname{Td}(\bar{f})) .
\end{aligned}
$$

Thus the result follows from the additivity.

We end this chapter with the relation between the singular Bott-Chern classes of Bismut-Gillet-Soule [12] and the theory of homogeneous analytic torsion classes. We draw attention to the difference of normalizations. Let us momentarily denote by $\tau$ the theory of singular Bott-Chern classes of Bismut-Gillet-Soulé. By the anomaly formulas, it may be extended to arbitrary embedded metrized complexes. Let $\bar{\xi}=(\bar{f}: X \rightarrow$ $\left.Y, \overline{\mathcal{F}}, \overline{f_{*} \mathcal{F}}\right)$ be a relative metrized complex, with $Y$ of dimension $d$. If $\tau^{(p-1, p-1)}$ denotes the component of degree $(p-1, p-1)$ of the current $\tau$, we define

$$
T^{B G S}(\bar{\xi})^{(2 p-1, p)}=-\frac{1}{2(2 \pi i)^{d-(p-1)}} \tau^{(p-1, p-1)} \in \widetilde{\mathcal{D}}_{D}^{2 p-1}\left(Y, N_{f}, p\right) .
$$

In the above equation, the factor $(2 \pi i)^{(p-1)}$ comes from the difference in the normalization of characteristic classes. In [12] the authors use real valued classes while we use twisted coefficients. The factor $(2 \pi i)^{d}$ comes from our convention about the Deligne complex of currents. The factor 2 comes from the fact that the second order differential operator that 
appears in the Deligne complex is $-2 \partial \bar{\partial}=2(2 \pi i) d d^{c}$, while the second order differential operator that appears in the differential equation considered by Bismut, Gillet and Soulé is $d d^{c}$. The main reason behind this change is that we want the Bott-Chern classes to be related to Beilinson's regulator and not to twice Beilinson's regulator (see [27] Theorem 3.5.4). Finally, the minus sign comes from the discrepancy of the differential equations of the singular Bott-Chern forms of Bismut-Gillet-Soulé and the analytic torsion forms of Bismut-Köhler. Note that we are forced to change this sign because we want to merge singular Bott-Chern forms and analytic torsion forms on a single theory. We put

$$
T^{B G S}(\bar{\xi})=\sum_{p \geq 1} T^{B G S}(\bar{\xi})^{(2 p-1, p)} \in \bigoplus_{p} \widetilde{\mathcal{D}}_{D}^{2 p-1}\left(Y, N_{f}, p\right) .
$$

We have the following comparison theorem [19, Thm. 9.25].

Theorem 3.21. For every embedded metrized complex $\xi$ we have

$$
T^{B G S}(\bar{\xi})=T^{h}(\bar{\xi})
$$

\section{Regular coherent sheaves}

In this section we recall some properties of regular sheaves. Let $X$ be a scheme and let $\mathbb{P}_{X}^{n}=\mathbb{P}_{X}(V)$ be the projective space of lines of the trivial bundle $V$ of rank $n+1$ on $X$. Let $\pi: \mathbb{P}_{X}^{n} \rightarrow X$ be the natural projection. By abuse of notation, if $\mathcal{G}$ is a sheaf on $X$, we will denote also by $\mathcal{G}$ the inverse image $\pi^{*} \mathcal{G}$.

Definition 4.1 ([38], Lecture 14). A quasi-coherent sheaf $\mathcal{F}$ on $\mathbb{P}_{X}^{n}$ is called regular if $R^{q} \pi_{*} \mathcal{F}(-q)=0$ for all $q>0$.

Recall the following properties of regular sheaves (see [39]).

(i) If $\mathcal{G}$ is a quasi-coherent sheaf on $X$, then $\mathcal{G} \otimes_{X} \mathcal{O}_{\mathbb{P}_{X}^{n}}(k)$ is regular for $k \geq 0$.

(ii) If the scheme $X$ is noetherian and $\mathcal{F}$ is a coherent sheaf on $\mathbb{P}_{X}^{n}$, then Serre's vanishing theorem implies that for $d$ large enough $\mathcal{F}(d)$ is regular.

(iii) Let $0 \rightarrow \mathcal{F}_{2} \rightarrow \mathcal{F}_{1} \rightarrow \mathcal{F}_{0} \rightarrow 0$ be an exact sequence of quasi-coherent sheaves on $\mathbb{P}_{X}^{n}$ and $d$ be an integer. Then

(a) if $\mathcal{F}_{2}(d)$ and $\mathcal{F}_{0}(d)$ are regular, then $\mathcal{F}_{1}(d)$ is regular;

(b) if $\mathcal{F}_{2}(d+1)$ and $\mathcal{F}_{1}(d)$ are regular, then $\mathcal{F}_{0}(d)$ is regular;

(c) if $\mathcal{F}_{0}(d)$ and $\mathcal{F}_{1}(d+1)$ are regular and the map $R^{0} \pi_{*}\left(\mathcal{F}_{1}(d)\right) \rightarrow R^{0} \pi_{*}\left(\mathcal{F}_{0}(d)\right)$ is surjective, then $\mathcal{F}_{2}(d+1)$ is regular.

(iv) If $\mathcal{F}$ is regular, then $\mathcal{F}(k)$ is regular for $k>0$.

(v) If $\mathcal{F}$ is regular, then the canonical map $R^{0} \pi_{*} \mathcal{F} \otimes_{X} \mathcal{O}_{\mathbb{P}_{X}^{n}} \rightarrow \mathcal{F}$ is surjective. 
Theorem 4.2 ([39, §8.1]). Let $\mathcal{F}$ be a regular quasi-coherent sheaf on $\mathbb{P}_{X}^{n}$. Then there exists a canonical resolution

$$
\gamma_{\text {can }}(\mathcal{F}): 0 \rightarrow \mathcal{G}_{n}(-n) \rightarrow \mathcal{G}_{n-1}(-n+1) \rightarrow \cdots \rightarrow \mathcal{G}_{0} \rightarrow \mathcal{F} \rightarrow 0
$$

where $\mathcal{G}_{i}(i=0, \ldots, n)$ are quasi-coherent sheaves on $X$. Moreover, for every $k \geq 0$, the sequence

$$
0 \rightarrow \mathcal{G}_{k} \rightarrow \mathcal{G}_{k-1} \otimes \operatorname{Sym}^{1} V^{\vee} \rightarrow \cdots \rightarrow \mathcal{G}_{0} \otimes \operatorname{Sym}^{k} V^{\vee} \rightarrow R^{0} \pi_{*}(\mathcal{F}(k)) \rightarrow 0
$$

is exact. Hence the sheaves $\mathcal{G}_{k}$ are determined by $\mathcal{F}$ up to unique isomorphism.

Corollary 4.3. Let $X$ be a noetherian scheme and $\mathcal{F}$ a coherent sheaf on $\mathbb{P}_{X}^{n}$. Then, for $d$ large enough, we have a resolution

$$
\gamma_{d}(\mathcal{F}): 0 \rightarrow \mathcal{G}_{n}(-n-d) \rightarrow \mathcal{G}_{n-1}(-n-d+1) \rightarrow \cdots \rightarrow \mathcal{G}_{0}(-d) \rightarrow \mathcal{F} \rightarrow 0
$$

where $\mathcal{G}_{i}, i=0, \ldots, n$ are coherent sheaves on $X$.

Example 4.4. The sheaf $\mathcal{O}(1)$ is regular. Its canonical resolution is

$$
0 \rightarrow \Lambda^{n+1} V^{\vee}(-n) \rightarrow \Lambda^{n} V^{\vee}(-n+1) \rightarrow \cdots \rightarrow \Lambda^{2} V^{\vee}(-1) \rightarrow V^{\vee} \rightarrow \mathcal{O}(1) \rightarrow 0 .
$$

Twisting this exact sequence by $\mathcal{O}(-1)$ we obtain the Koszul exact sequence

$$
0 \rightarrow \Lambda^{n+1} V^{\vee}(-n-1) \rightarrow \Lambda^{n} V^{\vee}(-n) \rightarrow \cdots \rightarrow \Lambda^{2} V^{\vee}(-2) \rightarrow V^{\vee}(-1) \rightarrow \mathcal{O} \rightarrow 0,
$$

that we denote $K$. We will denote by $K(k)$ its twist by $\mathcal{O}(k)$.

Theorem $4.5([50])$. (i) Let $\mathcal{F}$ be a regular coherent sheaf on $\mathbb{P}_{X}^{n}$, and let $\gamma_{\mathrm{can}}(\mathcal{F})$ be the canonical resolution of $\mathcal{F}$ as in Theorem 4.2 Let

$$
\varepsilon_{1}: 0 \rightarrow \mathcal{F}_{n+k}(-n-k) \rightarrow \cdots \rightarrow \mathcal{F}_{1}(-1) \rightarrow \mathcal{F}_{0} \rightarrow \mathcal{F} \rightarrow 0
$$

be an exact sequence of coherent sheaves, where the $\mathcal{F}_{i}$ are sheaves on $X$. Then there exist natural surjective morphisms of sheaves $\mathcal{F}_{i} \rightarrow \mathcal{G}_{i}$ on $X, 0 \leq i \leq n$ making commutative the diagram

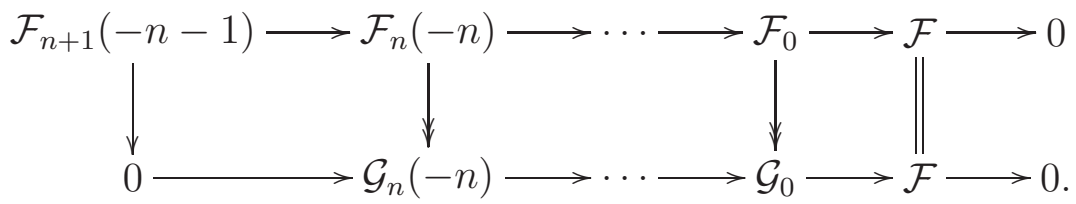

(ii) Let $\mathcal{F}$ be a regular coherent sheaf on $X$, and $\gamma_{\text {can }}(\mathcal{F})$ the canonical resolution. There exists a resolution of $\mathcal{F}(1)$ of the form

$$
\varepsilon_{2}: 0 \rightarrow \mathcal{S}_{n+k}(-n-k) \rightarrow \cdots \rightarrow \mathcal{S}_{1}(-1) \rightarrow \mathcal{S}_{0} \rightarrow \mathcal{F}(1) \rightarrow 0
$$

such that $\mathcal{S}_{0} \ldots, \mathcal{S}_{n+k}$ are coherent sheaves on $X$ and the following diagram of exact sequences with surjective vertical arrows is commutative:

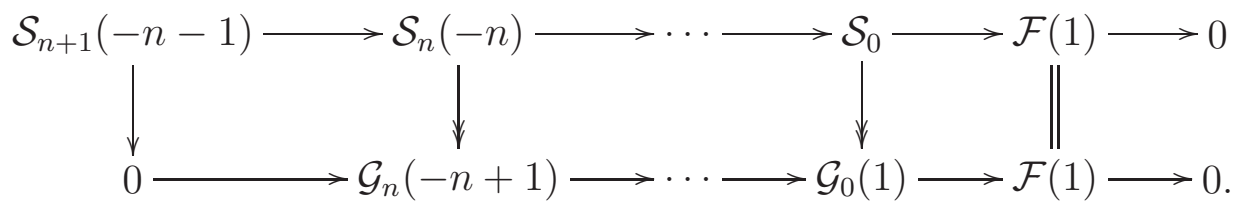


Proof. We introduce the sheaves $\mathcal{N}_{j}$ and $\mathcal{K}_{j}$ defined as the kernels at each term of the sequences $\gamma_{\text {can }}$ and $\varepsilon_{1}$, respectively. Hence, there are exact sequences

$$
\begin{aligned}
& 0 \rightarrow \mathcal{N}_{j+1}(j+1) \rightarrow \mathcal{G}_{j+1} \rightarrow \mathcal{N}_{j}(j+1) \rightarrow 0, \\
& 0 \rightarrow \mathcal{K}_{j+1}(j+1) \rightarrow \mathcal{F}_{j+1} \rightarrow \mathcal{K}_{j}(j+1) \rightarrow 0 .
\end{aligned}
$$

With these notations, observe that $\mathcal{N}_{-1}=\mathcal{K}_{-1}=\mathcal{F}$. By induction, starting from the left hand side of the long exact sequences, it is easily checked that $\mathcal{N}_{j}(j+1)$ and $\mathcal{K}_{j}(j+1)$ are regular sheaves, for $j \geq-1$. Also, by Theorem 4.2, we find that $\mathcal{G}_{j+1}=\pi_{*}\left(\mathcal{N}_{j}(j+1)\right)$ for $j \geq-1$. We next prove by induction that, for each $k \geq-1$, there is a commutative diagram of exact sequences

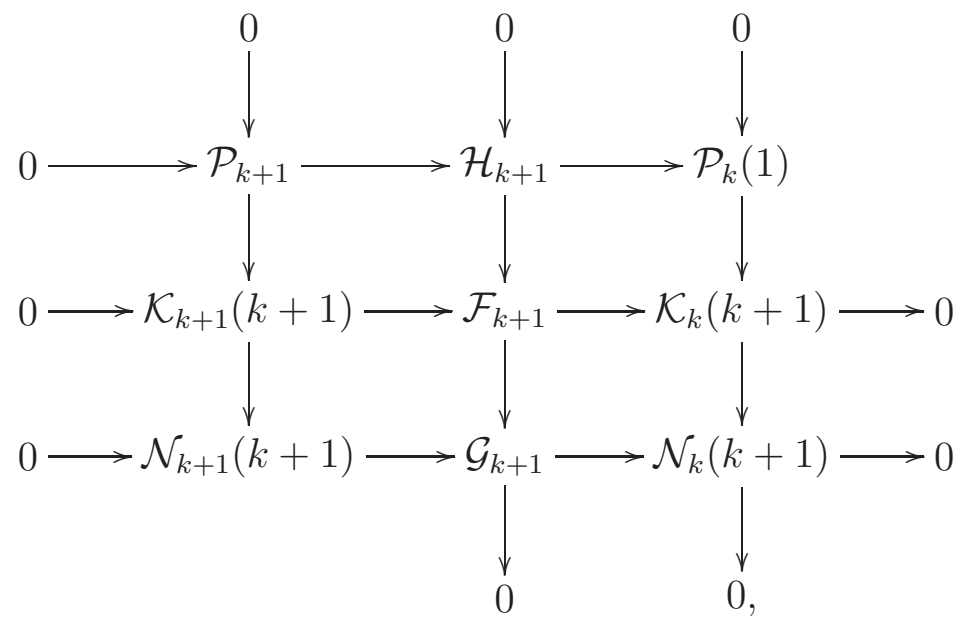

where $\mathcal{H}_{k+1}, \mathcal{P}_{k}$ and $\mathcal{P}_{k+1}$ are defined as the kernels of the corresponding morphisms, and that $\mathcal{P}_{k}(1)$ is regular. Assume that this is true for a fixed $k \geq 1$. In order to proceed with the induction, we need to prove: (a) the map $\mathcal{K}_{k+1}(k+2) \rightarrow \mathcal{N}_{k+1}(k+2)$ is surjective, (b) the sheaf $\mathcal{P}_{k+1}(1)$ is regular, and (c) there is an induced surjective map $\mathcal{F}_{k+2} \rightarrow \mathcal{G}_{k+2}$.

We first prove (a). If we apply $\pi_{*}$ to the last two columns of diagram (4.6). Observing that $\mathcal{F}_{k+1}, \mathcal{G}_{k+1}$ and $\mathcal{H}_{j+1}$ are actually sheaves on $X$ and recalling that $\mathcal{K}_{k+1}(k+2)$ is regular (so that $R^{1} \pi_{*} \mathcal{K}_{k+1}(k+1)=0$ ), we find a commutative diagram of exact sequences

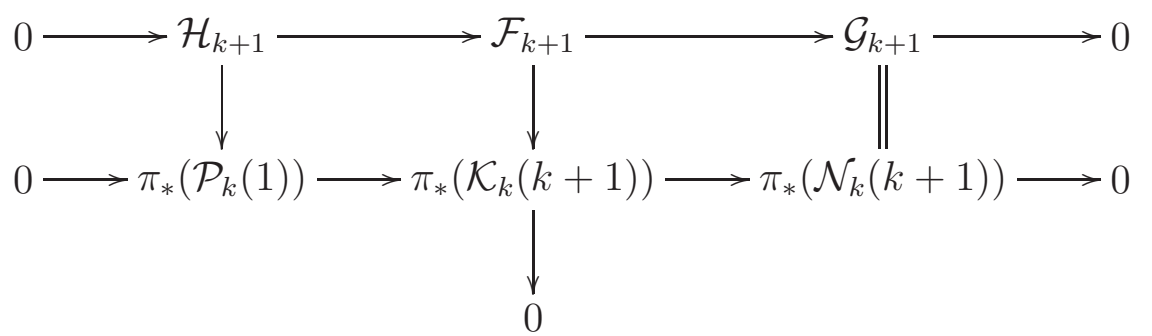

It follows that the map $\mathcal{H}_{k+1} \rightarrow \pi_{*}\left(\mathcal{P}_{k}(1)\right)$ is a surjection. Since $\mathcal{P}_{k}(1)$ is regular, we have that $\pi_{*}\left(\mathcal{P}_{k}(1)\right) \otimes \mathcal{O}_{\mathbb{P}_{X}^{n}} \rightarrow \mathcal{P}_{k}(1)$ is also a surjection. Thus the map $\mathcal{H}_{k+1} \rightarrow \mathcal{P}_{k}(1)$ is surjective. The diagram (4.6) implies that the map $\mathcal{K}_{k+1}(k+1) \rightarrow \mathcal{N}_{k+1}(k+1)$ is also surjective. Twisting by $\mathcal{O}(1)$, we obtain (a).

Now the regularity of $\mathcal{H}_{k+1}$ and $\mathcal{P}_{k}(1)$, and the surjectivity of $\mathcal{H}_{k+1} \rightarrow \pi_{*}\left(\mathcal{P}_{k}(1)\right)$ ensure the regularity of $\mathcal{P}_{k+1}(1)$. In its turn, this shows that the sequence

$$
0 \rightarrow \pi_{*}\left(\mathcal{P}_{k+1}(1)\right) \rightarrow \pi_{*}\left(\mathcal{K}_{k+1}(k+2)\right) \rightarrow \pi_{*}\left(\mathcal{N}_{k+1}(k+2)\right) \rightarrow 0
$$


is exact. Finally, we observe that there is a surjective map

$$
\mathcal{F}_{k+2} \longrightarrow \pi_{*}\left(\mathcal{K}_{k+1}(k+2)\right),
$$

by the regularity of $\mathcal{K}_{k+2}(k+3)$. From (4.7) and (4.8), we obtain a surjection

$$
\mathcal{F}_{k+2} \longrightarrow \pi_{*}\left(\mathcal{N}_{k+1}(k+2)\right)=\mathcal{G}_{k+2}
$$

This completes the proof of the inductive step. Note that the first step of the induction $(k=-1)$ is part of the data. From the existence of the diagrams we deduce (i).

To prove the second item we construct the resolution $\mathcal{S}_{*}$ inductively. We will denote by $\mathcal{K}_{k}$ the kernel of any map $\mathcal{S}_{k}(-k) \rightarrow \mathcal{S}_{k-1}(-k+1)$ already defined and by $\mathcal{N}_{k}$ the successive kernels of the canonical resolution of $\mathcal{F}$ as in the proof of the first statement.

Assume that we have constructed the sequence $\varepsilon_{2}$ up to $\mathcal{S}_{k}(-k)$ with the further conditions that $\mathcal{K}_{k}(k+1)$ is regular and that there is an exact sequence

$$
0 \rightarrow \mathcal{P}_{k}(1) \rightarrow \mathcal{K}_{k}(k+1) \rightarrow \mathcal{N}_{k}(k+2) \rightarrow 0
$$

with $\mathcal{P}_{k}(1)$ regular. We have to show that we can extend the resolution one step satisfying the same conditions. Recall that we already know that $\mathcal{N}_{k}(k+1)$ is regular. We consider as well the surjection $\mathcal{G}_{k+1}(1) \longrightarrow \mathcal{N}_{k}(k+2)$. We form the fiber product

$$
\mathcal{T}_{k+1}:=\operatorname{Ker}\left(\mathcal{K}_{k}(k+1) \oplus \mathcal{G}_{k+1}(1) \rightarrow \mathcal{N}_{k}(k+2)\right) .
$$

Observe that $\mathcal{T}_{k+1}$ is regular, because both $\mathcal{N}_{k}(k+1), \mathcal{K}_{k}(k+1) \oplus \mathcal{G}_{k+1}(1)$ are regular and the morphism

$$
\pi_{*}\left(\mathcal{K}_{k}(k) \oplus \mathcal{G}_{k+1}\right) \longrightarrow \mathcal{G}_{k+1}=\pi_{*}\left(\mathcal{N}_{k}(k+1)\right)
$$

is surjective. So are the arrows $\mathcal{T}_{k+1} \rightarrow \mathcal{G}_{k+1}(1)$ and $\mathcal{T}_{k+1} \rightarrow \mathcal{K}_{k}(k+1)$. Therefore, if we define $\mathcal{S}_{k+1}=\pi_{*}\left(\mathcal{T}_{k+1}\right)$, we have a commutative diagram of exact sequences

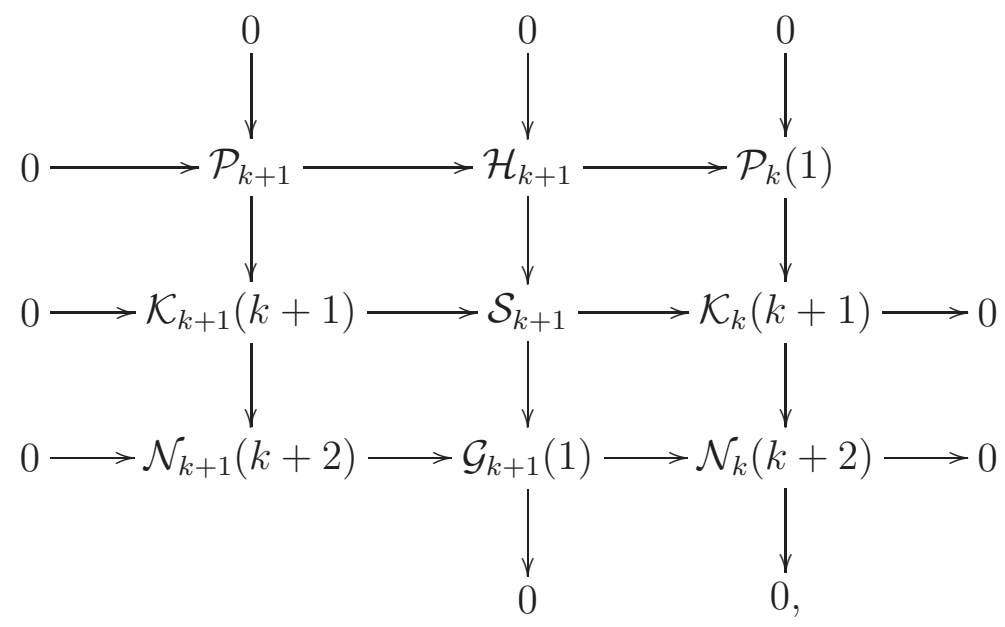

where $\mathcal{H}_{k+1}$ and $\mathcal{P}_{k+1}$ are defined as the kernels of the corresponding morphisms. Thus we have been able to extend the resolution one step further. We still need to show that this 
extension satisfies the extra properties. We observe that, by the definition of $\mathcal{S}_{k+1}$ and the left exactness of direct images, the map $\pi_{*}\left(\mathcal{S}_{k+1}\right) \rightarrow \pi_{*}\left(\mathcal{G}_{k+1}(1)\right)$ is surjective. Therefore $\mathcal{H}_{k+1}(1)$ is regular. Moreover, one can check that $\mathcal{S}_{k+1}$ is the fiber product

$$
\mathcal{S}_{k+1}=\operatorname{Ker}\left(\pi_{*}\left(\mathcal{G}_{k+1}(1)\right) \oplus \pi_{*}\left(\mathcal{K}_{k}(k+1)\right) \rightarrow \pi_{*}\left(\mathcal{N}_{k}(k+2)\right)\right) .
$$

This implies easily that $\pi_{*}\left(\mathcal{H}_{k+1}\right)=\pi_{*}\left(\mathcal{P}_{k}(1)\right)$. We also observe that, by definition of fiber product, $\mathcal{P}_{k}(1)=\operatorname{Ker}\left(\mathcal{T}_{k+1} \rightarrow \mathcal{G}_{k+1}(1)\right)$. Since $\mathcal{S}_{k+1}$ surjects onto $\mathcal{T}_{k+1}$, we deduce that the morphism $\mathcal{H}_{k+1} \rightarrow \mathcal{P}_{k}(1)$ is surjective. From this we conclude that the morphism $\mathcal{K}_{k+1}(k+2) \rightarrow \mathcal{N}_{k+1}(k+3)$ is surjective and that the sheaf $\mathcal{P}_{k+1}(1)$ is regular. Since $\mathcal{N}_{k+1}(k+3)$ is regular, we deduce that $\mathcal{K}_{k+1}(k+2)$ is regular. Therefore $\mathcal{S}_{\|+\infty}$ satisfies all the required properties, concluding the proof of (ii).

We end this section recalling the notion of generating class of a triangulated category.

Definition 4.9. Let $\mathrm{D}$ be a triangulated category. A generating class is a subclass $\mathrm{C}$ of $\mathrm{D}$ such that the smallest triangulated subcategory of $\mathbf{D}$ that contains $\mathbf{C}$ is equivalent to $\mathbf{D}$ via the inclusion.

A well-known direct consequence of Theorem 4.2 is the following result.

Corollary 4.10. The class of objects of the form $\mathcal{G}(k)$, with $\mathcal{G}$ a coherent sheaf in $X$ and $-n \leq k \leq 0$, is a generating class of $\mathrm{D}^{\mathrm{b}}\left(\mathbb{P}_{X}^{n}\right)$.

\section{Analytic torsion for projective spaces}

Let $n$ be a non-negative integer, $V$ the $n+1$ dimensional vector space $\mathbb{C}^{n+1}$ and $\mathbb{P}^{n}:=$ $\mathbb{P}^{n}(V)$ the projective space of lines in $V$. We write $\bar{V}$ for the vector space $V$ together with the trivial metric. We will denote by $V$ the trivial vector bundle of fiber $V$ over any base.

We may construct natural relative hermitian complexes that arise by considering the sheaves $\mathcal{O}(k)$, their cohomology and the Fubini-Study metric. If we endow the trivial sheaf with the trivial metric and $\mathcal{O}(1)$ with the Fubini-Study metric, then the tangent bundle $T_{\pi}$ carries a quotient hermitian structure via the short exact sequence

$$
0 \rightarrow \mathcal{O}_{\mathbb{P}_{\mathbb{C}}^{n}} \rightarrow \mathcal{O}(1)^{n+1} \rightarrow T_{\pi} \rightarrow 0
$$

We will denote the resulting hermitian vector bundle by $\bar{T}_{\pi}^{\mathrm{FS}}$ and call it the Fubini-Study metric of $T_{\pi}$. The arrow $\left(\pi, \bar{T}_{\pi}^{\mathrm{FS}}\right)$ in $\overline{\mathbf{S m}}_{* / \mathbb{C}}$ will be written $\bar{\pi}^{\mathrm{FS}}$. We endow the invertible sheaves $\mathcal{O}(k)$ with the $k$-th tensor power of the Fubini-Study metric on $\mathcal{O}(1)$. We refer to them by $\overline{\mathcal{O}(k)}$.

We now describe natural hermitian structures on the complexes $\pi_{*} \mathcal{O}(k)$. First assume $k \geq 0$. The sheaf $\mathcal{O}(k)$ is $\pi$-acyclic, hence $\pi_{*} \mathcal{O}(k)=\mathrm{H}^{0}\left(\mathbb{P}_{\mathbb{C}}^{n}, \mathcal{O}(k)\right)$ as a complex concentrated in degree 0 . This space is naturally equipped with the $L^{2}$ metric with respect to the Fubini-Study metric on $\mathcal{O}(k)$ and the volume form $\mu=c_{1}(\overline{\mathcal{O}(1)})^{\wedge n} / n$ ! on $\mathbb{P}_{\mathbb{C}}^{n}$. Namely, given global sections $s, t$ of $\mathcal{O}(k)$,

$$
\langle s, t\rangle_{L^{2}}=\int_{\mathbb{P}_{\mathbb{C}}^{n}}\langle s(x), t(x)\rangle_{x} \mu(x) .
$$


If $-n \leq k<0$, then $\pi_{*} \mathcal{O}(k)=0$ and we put the trivial metric on it. Finally, let $k \leq-n-1$. Then the cohomology of $\pi_{*} \mathcal{O}(k)$ is concentrated in degree $n$ and there is an isomorphism,

$$
\pi_{*} \mathcal{O}(k) \cong \mathrm{H}^{0}\left(\mathbb{P}_{\mathbb{C}}^{n}, \mathcal{O}(-k-n-1)\right)^{\vee}[-n] .
$$

Notice that this isomorphism is canonical due to Grothendieck duality and to the natural identification $\omega_{\mathbb{P}_{\mathbb{C}}^{n}}=\mathcal{O}(-n-1)$. Hence we may endow $\pi_{*} \mathcal{O}(k)$ with the dual of the $L^{2}$ metric on $\mathrm{H}^{0}\left(\mathbb{P}_{\mathbb{C}}^{n}, \mathcal{O}(-k-n-1)\right)$.

Notation 5.2. For every integer $k$, we introduce the relative metrized complex

$$
\overline{\xi_{n}}(k)=\left(\bar{\pi}^{\mathrm{FS}}, \overline{\mathcal{O}(k)}, \overline{\pi_{*} \mathcal{O}(k)}\right) .
$$

If $X$ is a smooth complex variety, we will also denote by $\bar{\xi}_{n}(k)$ its pull-back to $\mathbb{P}_{X}^{n}$. Let $\overline{\mathcal{F}}$ be a metrized coherent sheaf on $X$. Then we define $\overline{\mathcal{F}}(k)$ and $\overline{\pi_{*} \mathcal{F}(k)}$ by the equality

$$
\bar{\xi}_{n}(k) \otimes \overline{\mathcal{F}}=\left(\bar{\pi}^{\mathrm{FS}}, \overline{\mathcal{F}}(k), \overline{\pi_{*} \mathcal{F}(k)}\right) .
$$

Definition 5.4. Let $X$ be a complex smooth variety and $\pi: \mathbb{P}_{X}^{n} \rightarrow X$ the projection. An analytic torsion class for the relative hermitian complex $\bar{\xi}=\left(\bar{\pi}, \overline{\mathcal{F}}, \overline{\pi_{*} \mathcal{F}}\right)$ is a class $\widetilde{\eta} \in \bigoplus_{p} \widetilde{\mathcal{D}}^{2 p-1}(X, p)$ such that

$$
\mathrm{d}_{\mathcal{D}} \widetilde{\eta}=\operatorname{ch}\left(\overline{\pi_{*} \mathcal{F}}\right)-\bar{\pi}_{b}[\operatorname{ch}(\overline{\mathcal{F}})] .
$$

The existence of this class is guaranteed by the Grothendieck-Riemann-Roch theorem, which implies that the two currents at the right hand side of equation (5.5) are cohomologous. Since the map $\pi$ is smooth, the analytic torsion class is the class of a smooth form.

Definition 5.6. Let $n$ be a non-negative integer. A theory of analytic torsion classes for projective spaces of dimension $n$ is an assignment that, to each relative metrized complex $\bar{\xi}=\left(\bar{\pi}: \mathbb{P}_{X}^{n} \rightarrow X, \overline{\mathcal{F}}, \overline{\pi_{*} \mathcal{F}}\right)$ of relative dimension $n$, assigns a class of differential forms

$$
T(\bar{\xi}) \in \bigoplus_{p} \widetilde{\mathcal{D}}^{2 p-1}(X, p),
$$

satisfying the following properties.

(i) (Differential equation) $\mathrm{d}_{\mathcal{D}} T(\bar{\xi})=\operatorname{ch}\left(\overline{\pi_{*} \mathcal{F}}\right)-\bar{\pi}_{b}[\operatorname{ch}(\overline{\mathcal{F}})]$.

(ii) (Functoriality) Given a morphism $f: Y \longrightarrow X$, we have $T\left(f^{*} \bar{\xi}\right)=f^{*} T(\bar{\xi})$.

(iii) (Additivity and normalization) If $\bar{\xi}_{1}$ and $\bar{\xi}_{2}$ are relative metrized complexes on $X$, then $T\left(\bar{\xi}_{1} \oplus \bar{\xi}_{2}\right)=T\left(\bar{\xi}_{1}\right)+T\left(\bar{\xi}_{2}\right)$.

(iv) (Projection formula) For any hermitian vector bundle $\bar{G}$ on $X$, and any integer $k \in[-n, 0]$, we have $T\left(\bar{\xi}_{n}(k) \otimes \bar{G}\right)=T\left(\bar{\xi}_{n}(k)\right) \bullet \operatorname{ch}(\bar{G})$.

A theory of analytic torsion classes for projective spaces is an assignment as before, for all non-negative integers $n$. 
Definition 5.7. Let $T$ be a theory of analytic torsion classes for projective spaces of dimension $n$. Fix as base space the point Spec $\mathbb{C}$. The characteristic numbers of $T$ are

$$
t_{n, k}(T):=T\left(\bar{\xi}_{n}(k)\right) \in \widetilde{\mathcal{D}}^{1}(\operatorname{Spec} \mathbb{C}, 1)=\mathbb{R}, k \in \mathbb{Z} .
$$

The numbers $t_{n, k}(T),-n \leq k \leq 0$ will be called the main characteristic numbers of $T$.

The central result of this section is the following classification theorem.

Theorem 5.9. Let $n$ be a non-negative integer and let $\mathfrak{t}=\left(t_{n, k}\right)_{k=-n, \ldots, 0}$ be a family of arbitrary real numbers. Then there exists a unique theory $T_{\mathfrak{t}}$ of analytic torsion classes for projective spaces of dimension $n$, such that $t_{n, k}\left(T_{\mathfrak{t}}\right)=t_{n, k}$.

Before proving Theorem 5.9, we show some consequences of the definition of the analytic torsion classes. First we state some anomaly formulas that determine the dependence of the analytic torsion classes with respect to different choices of metrics.

Proposition 5.10. Let $T$ be a theory of analytic torsion classes for projective spaces of dimension $n$. Let $\bar{\xi}=\left(\bar{\pi}: \mathbb{P}_{X}^{n} \rightarrow X, \overline{\mathcal{F}}, \overline{\pi_{*} \mathcal{F}}\right)$ be a relative metrized complex.

(i) If $\overline{\mathcal{F}}^{\prime}$ is another choice of metric on $\mathcal{F}$ and $\bar{\xi}_{1}=\left(\bar{\pi}: \mathbb{P}_{X}^{n} \rightarrow X, \overline{\mathcal{F}}^{\prime}, \overline{\pi_{*} \mathcal{F}}\right)$, then

$$
T\left(\bar{\xi}_{1}\right)=T(\bar{\xi})+\bar{\pi}_{b}\left[\widetilde{\operatorname{ch}}\left(\overline{\mathcal{F}}^{\prime}, \overline{\mathcal{F}}\right)\right] .
$$

(ii) If $\bar{\pi}^{\prime}$ is another hermitian structure on $\pi$ and $\bar{\xi}_{2}=\left(\bar{\pi}^{\prime}: \mathbb{P}_{X}^{n} \rightarrow X, \overline{\mathcal{F}}, \overline{\pi_{*} \mathcal{F}}\right)$, then

$$
T\left(\bar{\xi}_{2}\right)=T(\bar{\xi})+\bar{\pi}_{b}^{\prime}\left[\operatorname{ch}(\overline{\mathcal{F}}) \bullet \widetilde{T d}_{m}\left(\bar{\pi}^{\prime}, \bar{\pi}\right)\right]
$$

(iii) If ${\overline{\pi_{*} \mathcal{F}}}^{\prime}$ is another choice of metric on $\pi_{*} \mathcal{F}$, and $\bar{\xi}_{3}=\left(\bar{\pi}: \mathbb{P}_{X}^{n} \rightarrow X, \overline{\mathcal{F}},{\overline{\pi_{*} \mathcal{F}^{\prime}}}^{\prime}\right)$, then

$$
T\left(\bar{\xi}_{3}\right)=T(\bar{\xi})-\widetilde{\operatorname{ch}}\left({\overline{\pi_{*} \mathcal{F}}}^{\prime}, \overline{\pi_{*} \mathcal{F}}\right) .
$$

Proof. The proof is the same as the proof of Proposition 3.17

Next we state the behavior of analytic torsion classes for projective spaces with respect to distinguished triangles.

Proposition 5.12. Let $T$ be a theory of analytic torsion classes for projective spaces of dimension $n$. Let $X$ be a smooth complex variety and $\pi: \mathbb{P}_{X}^{n} \rightarrow X$ the projection. Consider distinguished triangles in $\overline{\mathbf{D}}^{\mathrm{b}}\left(\mathbb{P}_{X}^{n}\right)$ and $\overline{\mathbf{D}}^{\mathrm{b}}(X)$ respectively:

$$
(\bar{\tau}): \overline{\mathcal{F}}_{2} \rightarrow \overline{\mathcal{F}}_{1} \rightarrow \overline{\mathcal{F}}_{0} \rightarrow \overline{\mathcal{F}}_{2}[1] \text { and }\left(\overline{\pi_{*} \tau}\right): \bar{\pi}_{*} \mathcal{F}_{2} \rightarrow \bar{\pi}_{*} \overline{\mathcal{F}}_{1} \rightarrow \bar{\pi}_{*} \mathcal{F}_{0} \rightarrow \bar{\pi}_{*} \mathcal{F}_{2}[1]
$$

and define relative metrized complexes $\bar{\xi}_{i}=\left(\bar{\pi}, \overline{\mathcal{F}}_{i}, \bar{\pi}_{*} \mathcal{F}_{i}\right), i=0,1,2$. Then

$$
\sum_{j}(-1)^{j} T\left(\bar{\xi}_{j}\right)=\widetilde{\operatorname{ch}}\left(\overline{\pi_{*} \tau}\right)-\bar{\pi}_{b}(\widetilde{\operatorname{ch}}(\bar{\tau})) .
$$

Proof. The proof is similar to that of 3.19 . 
In view of this proposition, we see that the additivity axiom is equivalent to the apparently stronger statement of the next corollary.

Corollary 5.13. With the assumptions of Proposition 5.12 if $\bar{\tau}$ and $\overline{\pi_{*} \tau}$ are tightly distinguished, then $T\left(\bar{\xi}_{1}\right)=T\left(\bar{\xi}_{0}\right)+T\left(\bar{\xi}_{2}\right)$.

Corollary 5.14. Let $\bar{\xi}=\left(\bar{\pi}, \overline{\mathcal{F}}, \overline{\pi_{*} \mathcal{F}}\right)$ be a relative metrized complex and let $\bar{\xi}[i]=$ $\left(\bar{\pi}, \overline{\mathcal{F}}[i], \overline{\pi_{*} \mathcal{F}}[i]\right)$ be the shifted relative metrized complex. Then $T(\bar{\xi})=(-1)^{i} T(\bar{\xi}[i])$.

Proof. It is enough to treat the case $i=1$. We consider the tightly distinguished triangle

$$
\overline{\mathcal{F}} \rightarrow \overline{\operatorname{cone}}\left(\operatorname{id}_{\overline{\mathcal{F}}}\right)-\rightarrow \overline{\mathcal{F}}[1]-\rightarrow
$$

and the analogous triangle for direct images. Since $\overline{\operatorname{cone}}\left(\mathrm{id}_{\overline{\mathcal{F}}}\right)$ and $\overline{\mathrm{cone}}\left(\mathrm{id} \overline{\pi_{*} \mathcal{F}}\right)$ are meager, we have, by the anomaly formulas and the additivity axiom,

$$
T\left(\bar{\pi}, \overline{\operatorname{cone}}\left(\mathrm{id}_{\overline{\mathcal{F}}}\right), \overline{\operatorname{cone}}\left(\mathrm{id}_{\overline{\pi_{*} \mathcal{F}}}\right)\right)=T(\bar{\pi}, \overline{0}, \overline{0})=0 .
$$

Hence, the result follows from Corollary 5.13

Next we rewrite Proposition 5.12 in the language of complexes of metrized coherent sheaves. Let

$$
\bar{\varepsilon}: \quad 0 \rightarrow \overline{\mathcal{F}}_{m} \rightarrow \cdots \rightarrow \overline{\mathcal{F}}_{l} \rightarrow 0
$$

be a bounded complex of metrized coherent sheaves on $\mathbb{P}_{X}^{n}$ and assume that hermitian structures on the complexes $\pi_{*} \mathcal{F}_{j}, j=l, \ldots, m$ are chosen. Let $[\bar{\varepsilon}],\left[\overline{\pi_{*} \varepsilon}\right] \in \mathrm{Ob}_{\overline{\mathbf{D}}}^{\mathrm{b}}\left(\mathbb{P}_{X}^{n}\right)$ be the associated objects as in [17, Def. 3.37, Def. 3.39].

Corollary 5.15. With the above hypothesis,

$$
T\left(\bar{\pi},[\bar{\varepsilon}],\left[\overline{\pi_{*} \varepsilon}\right]\right)=\sum_{j=l}^{m}(-1)^{j} T\left(\bar{\pi}, \overline{\mathcal{F}}_{j}, \overline{\pi_{*} \mathcal{F}_{j}}\right) .
$$

Moreover, if $\varepsilon$ is acyclic, then $T\left(\bar{\pi},[\bar{\varepsilon}],\left[\overline{\pi_{*} \varepsilon}\right]\right)=\widetilde{\operatorname{ch}}\left(\overline{\pi_{*} \varepsilon}\right)-\bar{\pi}_{b}[\widetilde{c h}(\bar{\varepsilon})]$.

Finally, we show that the projection formula holds in greater generality:

Proposition 5.16. Let $T$ be a theory of analytic torsion classes for projective spaces of dimension $n$. Let $X$ be a smooth complex variety, let $\bar{\xi}=\left(\bar{\pi}, \overline{\mathcal{F}}, \overline{\pi_{*} \mathcal{F}}\right)$ be a relative metrized complex and let $\overline{\mathcal{G}}$ be an object in $\overline{\mathbf{D}}^{\mathrm{b}}(X)$. Then

$$
T(\bar{\xi} \otimes \overline{\mathcal{G}})=T(\bar{\xi}) \bullet \operatorname{ch}(\overline{\mathcal{G}})
$$

Proof. By the anomaly formulas, if equation (5.17) holds for a particular choice of hermitian structures on $\pi, \mathcal{F}$ and $\pi_{*} \mathcal{F}$ then it holds for any other choice. Moreover, if we are in the situation of Proposition 5.12 and equation (5.17) holds for two of $\bar{\xi}_{0}, \bar{\xi}_{1}, \bar{\xi}_{2}$, then it holds for the third. Using that the objects of the form $\mathcal{H}(k)$, where $\mathcal{H}$ is a coherent sheaf on $X$ and $k=-n, \ldots, 0$, constitute a generating class of $\mathbf{D}^{\mathrm{b}}\left(\mathbb{P}_{X}^{n}\right)$, we are reduced to prove that

$$
T\left(\bar{\xi}_{n}(k) \otimes \overline{\mathcal{G}}\right)=T\left(\bar{\xi}_{n}(k)\right) \bullet \operatorname{ch}(\overline{\mathcal{G}}) .
$$


for $k=-n, \ldots, 0$. Now, if

$$
\overline{\mathcal{G}}_{2}-\rightarrow \overline{\mathcal{G}}_{1}-\rightarrow \overline{\mathcal{G}}_{0}-\rightarrow
$$

is a distinguished triangle in $\overline{\mathbf{D}}^{\mathrm{b}}(X)$ and equation (5.17) is satisfied for two of $\overline{\mathcal{G}}_{2}, \overline{\mathcal{G}}_{1}$, $\overline{\mathcal{G}}_{0}$, then it is satisfied also by the third. Therefore, since the complexes of vector bundles concentrated in a single degree constitute a generating class of $\mathbf{D}^{\mathrm{b}}(X)$, the projection formula axiom implies the proposition.

Proof of Theorem 5.9. To begin with, we prove the uniqueness assertion. Assume a theory of analytic torsion classes $T$, with main characteristic numbers $t_{n, k},-n \leq k \leq 0$, exists. Then, the anomaly formulas (Proposition 5.10) imply that, if $T\left(\bar{\pi}, \overline{\mathcal{F}}, \overline{\pi_{*} \mathcal{F}}\right)$ is known for a particular choice of hermitian structures on $\pi, \mathcal{F}$ and $\pi_{*} \mathcal{F}$ then the value of $T\left(\bar{\pi}^{\prime}, \overline{\mathcal{F}}^{\prime},{\overline{\pi_{*}}}^{\prime}\right)$ for any other choice of hermitian structures is fixed. By Proposition 5.12 if we know the value of $T\left(\bar{\pi}, \overline{\mathcal{F}}, \overline{\pi_{*} \mathcal{F}}\right)$, for $\mathcal{F}$ in a generating class, then $T$ is determined. By the projection formula (Proposition 5.16), the characteristic numbers determine the values of $T(\bar{\xi}(k) \otimes \mathcal{G}), k=-n, \ldots, 0$. Finally, since by Corollary 4.10, the objects of the form $\mathcal{G}(k), k=-n, \ldots, 0$ form a generating class, we deduce that the characteristic numbers determine the theory $T$. Thus, if it exists, the theory $T_{\mathfrak{t}}$ is unique.

In particular, from the above discussion we see that the main characteristic numbers determine all the characteristic numbers. We now derive an explicit inductive formula for them. Consider the metrized Koszul resolution

$$
\bar{K}: 0 \rightarrow \Lambda^{n+1} \bar{V}^{\vee}(-n-1) \rightarrow \cdots \rightarrow \Lambda^{1} \bar{V}^{\vee}(-1) \rightarrow \overline{\mathcal{O}}_{\mathbb{P}_{\mathbb{C}}^{n}} \rightarrow 0,
$$

where $\mathcal{O}(k)$, for $k \neq 0$, has the Fubini-Study metric and $\overline{\mathcal{O}}_{\mathbb{P}_{\mathbb{C}}^{n}}$ has the trivial metric. We will denote by $\bar{K}(k)$ the above exact sequence twisted by $\overline{\mathcal{O}(k)}, k \in \mathbb{Z}$, again with the Fubini-Study metric. Recall the definition of the relative metrized complexes $\bar{\xi}_{n}(k)$ (5.3). In particular, for every $k$, we have fixed natural hermitian structures on the objects $\pi_{*} \mathcal{O}(k-j)$. According to [17, Def. 3.37, Def. 3.39], we may consider the classes $[\bar{K}(k)]$ and $\left[\overline{\pi_{*} K(k)}\right]$ in $\overline{\mathbf{D}}^{\mathrm{b}}\left(\mathbb{P}_{\mathbb{C}}^{n}\right)$ and $\overline{\mathbf{D}}^{\mathrm{b}}$ (Spec $\left.\mathbb{C}\right)$, respectively. By Corollary 5.15 , for each $k \in \mathbb{Z}$ we find

$$
\sum_{j=0}^{n+1}(-1)^{j} T\left(\bar{\xi}_{n}(k-j) \otimes \Lambda^{j} \bar{V}^{\vee}\right)=\widetilde{\operatorname{ch}}\left(\overline{\pi_{*} K(k)}\right)-\bar{\pi}_{b}^{\mathrm{FS}}[\widetilde{\operatorname{ch}}(\bar{K}(k))]
$$

Because $\Lambda^{j} \bar{V}^{\vee}$ is isometric to $\mathbb{C}^{\left(\begin{array}{c}n+1 \\ j\end{array}\right)}$ with the trivial metric, the additivity axiom for the theory $T$ and the definition of the characteristic numbers $t_{n, k-j}$ provide

$$
T\left(\bar{\xi}_{n}(k-j) \otimes \Lambda^{j} \bar{V}^{\vee}\right)=t_{n, k-j}\left(\begin{array}{c}
n+1 \\
j
\end{array}\right) .
$$

Therefore we derive

$$
\sum_{j=0}^{n+1}(-1)^{j}\left(\begin{array}{c}
n+1 \\
j
\end{array}\right) t_{n, k-j}=\widetilde{\operatorname{ch}}\left(\overline{\pi_{*} K(k)}\right)-\bar{\pi}_{b}^{\mathrm{FS}}[\widetilde{\operatorname{ch}}(\bar{K}(k))] .
$$


This equation gives us an inductive formula for all the characteristic numbers $t_{n, k}$ once we have fixed $n+1$ consecutive characteristic numbers and, in particular, once we have fixed the main characteristic numbers.

To prove the existence, we follow the proof of the uniqueness to obtain a formula for $T(\bar{\xi})$. We start with the main characteristic numbers $\mathfrak{t}=\left(t_{n, k}\right)_{-n \leq k \leq 0}$. We define the characteristic numbers $t_{n, k}$ for $k \in \mathbb{Z}$ inductively using equation (5.19).

We will need the following results.

Lemma 5.20. Let

$$
\bar{\eta}: 0 \rightarrow \overline{\mathcal{F}}_{2} \rightarrow \overline{\mathcal{F}}_{1} \rightarrow \overline{\mathcal{F}}_{0} \rightarrow 0
$$

be a short exact sequence of metrized coherent sheaves on $X$. Let $k$ be an integer, and $\overline{\mathcal{F}}(k)$ and $\overline{\pi_{*} \mathcal{F}(k)}$ be as in Notation 5.2 Thus we have an exact sequence $\bar{\eta}(k)$ of metrized coherent sheaves on $\mathbb{P}_{X}^{n}$ and a distinguished triangle $\overline{\pi_{*} \eta(k)}$. Then

$$
\widetilde{\operatorname{ch}}\left(\overline{\pi_{*} \eta(k)}\right)=\bar{\pi}_{b}^{\mathrm{FS}}(\widetilde{\operatorname{ch}}(\bar{\eta}(k)))
$$

Proof. By the Riemann-Roch theorem for the map $\mathbb{P}_{\mathbb{C}}^{n} \rightarrow$ Spec $\mathbb{C}$ we have

$$
\operatorname{ch}\left(\overline{\pi_{*} \mathcal{O}(k)}\right)=\pi_{*}\left(\operatorname{ch}(\overline{\mathcal{O}(k)}) \operatorname{Td}\left(\bar{\pi}^{\mathrm{FS}}\right)\right)
$$

Hence, by the properties of Bott-Chern classes and the choice of metrics

$$
\begin{aligned}
\widetilde{\operatorname{ch}}\left(\overline{\pi_{*} \eta(k)}\right) & =\widetilde{\operatorname{ch}}(\bar{\eta}) \bullet \operatorname{ch}\left(\overline{\pi_{*} \mathcal{O}(k)}\right) \\
& =\widetilde{\operatorname{ch}}(\bar{\eta}) \bullet \pi_{*}\left(\operatorname{ch}(\overline{\mathcal{O}(k)}) \operatorname{Td}\left(\bar{\pi}^{\mathrm{FS}}\right)\right) \\
& =\pi_{*}\left(\widetilde{\operatorname{ch}}(\bar{\eta}(k)) \bullet \operatorname{Td}\left(\bar{\pi}^{\mathrm{FS}}\right)\right) \\
& =\bar{\pi}_{b}^{\mathrm{FS}}(\widetilde{\operatorname{ch}}(\bar{\eta}(k))) .
\end{aligned}
$$

Lemma 5.23. Let

$$
\bar{\mu}: 0 \rightarrow \overline{\mathcal{M}}_{m}(-m-d) \rightarrow \cdots \rightarrow \overline{\mathcal{M}}_{l}(-l-d) \rightarrow 0
$$

be an exact sequence of metrized coherent sheaves on $\mathbb{P}_{X}^{n}$, where, for each $i=l, \ldots, m$, $\overline{\mathcal{M}}_{i}$ is a metrized coherent sheaf on $X$, and $\overline{\mathcal{M}}_{i}(k)$ is as in Notation 5.2 On $\pi_{*} \mathcal{M}_{i}(k)$ we consider the hermitian structures given also by Notation 5.2. Then

$$
\sum_{i=l}^{m}(-1)^{i} t_{n,-d-i} \operatorname{ch}\left(\overline{\mathcal{M}}_{i}\right)=\widetilde{\operatorname{ch}}\left(\overline{\pi_{*} \mu}\right)-\bar{\pi}_{b}^{\mathrm{FS}}(\widetilde{\operatorname{ch}}(\bar{\mu})) .
$$


Proof. We consider a commutative diagram of exact sequences

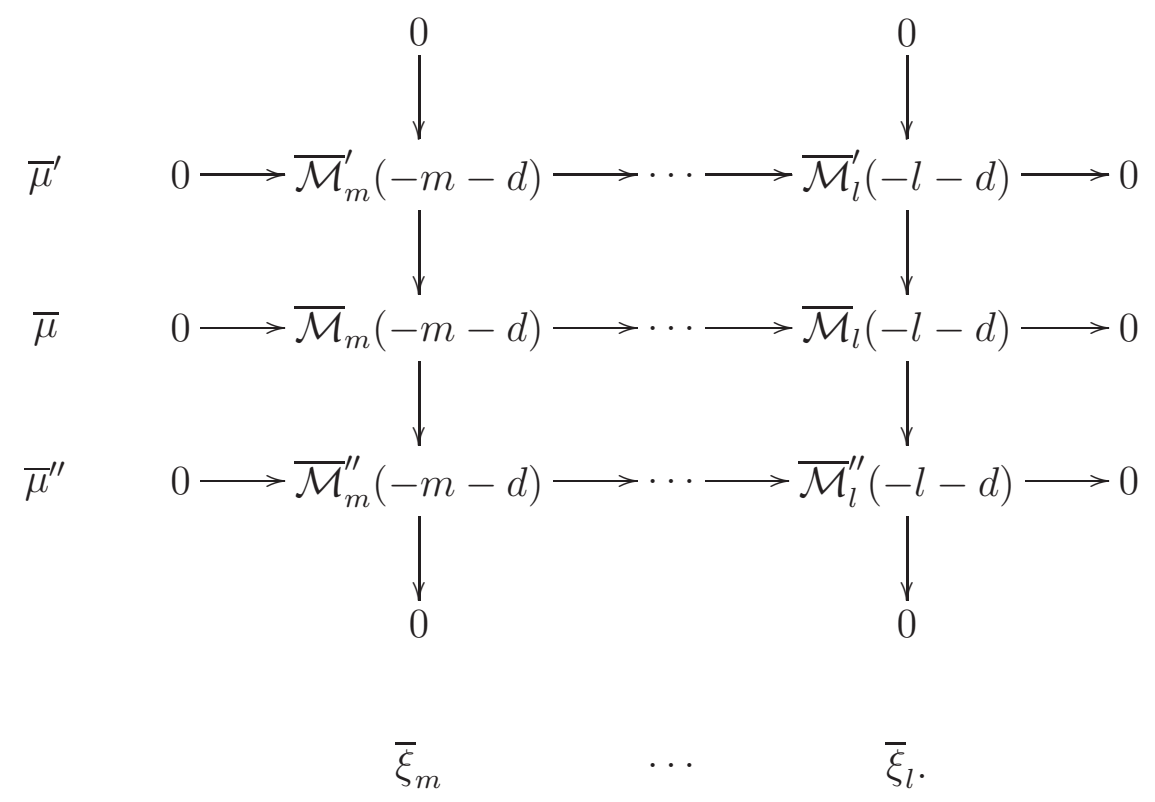

Claim. If equation (5.25) holds for two of $\bar{\mu}, \bar{\mu}^{\prime}$ and $\bar{\mu}^{\prime \prime}$, then it holds for the third.

Proof of the claim. On the one hand we have

$$
\sum_{i=l}^{m}(-1)^{i} t_{n,-d-i}\left(\operatorname{ch}\left(\overline{\mathcal{M}}_{i}^{\prime}\right)-\operatorname{ch}\left(\overline{\mathcal{M}}_{i}\right)+\operatorname{ch}\left(\overline{\mathcal{M}}_{i}^{\prime \prime}\right)\right)=\sum_{i=l}^{m}(-1)^{i} t_{n,-d-i} \mathrm{~d}_{\mathcal{D}} \widetilde{\operatorname{ch}}\left(\bar{\xi}_{i}\right) .
$$

But, if $t \in \mathcal{D}^{1}(\operatorname{Spec} \mathbb{C}, 1)=\mathbb{R}$ is a real number, in the group $\bigoplus_{p} \widetilde{\mathcal{D}}^{2 p-1}(X, p)$, we have

$$
t \mathrm{~d}_{\mathcal{D}} \widetilde{\operatorname{ch}}\left(\bar{\xi}_{i}\right)=-\mathrm{d}_{\mathcal{D}}\left(t \bullet \widetilde{\operatorname{ch}}\left(\bar{\xi}_{i}\right)\right)=0 .
$$

On the other hand, by Lemma 5.20

$$
\tilde{\operatorname{ch}}\left(\overline{\pi_{*} \mu^{\prime}}\right)-\widetilde{\operatorname{ch}}\left(\overline{\pi_{*} \mu}\right)+\widetilde{\operatorname{ch}}\left(\overline{\pi_{*} \mu^{\prime \prime}}\right)=\pi_{b}^{\mathrm{FS}}\left(\widetilde{\operatorname{ch}}\left(\bar{\mu}^{\prime}\right)\right)-\pi_{b}^{\mathrm{FS}}(\tilde{\operatorname{ch}}(\bar{\mu}))+\pi_{b}^{\mathrm{FS}}\left(\widetilde{\operatorname{ch}}\left(\bar{\mu}^{\prime \prime}\right)\right) .
$$

The proof of the lemma is done by induction on the length $r=m-l$ of the complex. If $r \leq n$ then $\mu(d+l)$ has the same shape as the canonical resolution of the zero sheaf. By the uniqueness of the canonical resolution, we have $\mathcal{M}_{i}=0$, for $i=l, \ldots, m$. Using the above claim when $\mathcal{M}_{i}=0$ has a non-trivial metric, we obtain the lemma for $r \leq n$.

Assume now that $r>n$. Let $K$ be the Koszul exact sequence (5.18). Then $K(1) \otimes \mathcal{M}_{l}$ is the canonical resolution of the regular coherent sheaf $\mathcal{M}_{l}(1)$. By Theorem 4.5(i) there is a surjection of exact sequences $\mu \rightarrow K(-l-d) \otimes \mathcal{M}_{l}$ whose kernel is an exact sequence

$$
\mu^{\prime}: 0 \rightarrow \mathcal{M}_{m}^{\prime}(-m-d) \rightarrow \cdots \rightarrow \mathcal{M}_{l+1}^{\prime}(-d-l-1) \rightarrow 0 .
$$

We consider on $K$ the metrics of (5.18), for $i=l+1, \ldots, m$, we choose arbitrary metrics on $\mathcal{M}_{i}^{\prime}$ and denote by $\bar{\mu}^{\prime}$ the corresponding exact sequence of metrized coherent sheaves.

By induction hypothesis, $\bar{\mu}^{\prime}$ satisfies equation (5.25). Moreover, since the characteristic numbers $t_{n, k}$ for $k \notin[0, n]$ are defined using equation (5.19), the exact sequence $\bar{K}(-l-$ d) $\otimes \overline{\mathcal{M}}_{l}$ also satisfies equation (5.25). Hence the lemma follows from the previous claim. 
We now treat the case of complexes concentrated in a single degree. Let $\overline{\mathcal{F}}$ be a coherent sheaf on $\mathbb{P}_{X}^{n}$ with a hermitian structure and let $\overline{\pi_{*} \mathcal{F}}$ be a choice of a hermitian structure on the direct image complex. Write $\bar{\xi}=\left(\bar{\pi}^{\mathrm{FS}}, \overline{\mathcal{F}}, \overline{\pi_{*} \mathcal{F}}\right)$ for the corresponding relative metrized complex.

Choose an integer $d$ such that $\mathcal{F}(d)$ is regular. Then we have the resolution $\gamma_{d}(\mathcal{F})$ of Corollary 4.3. More generally, let $\mu$ be an exact sequence of the form

$$
0 \rightarrow \mathcal{S}_{m}(-d-m) \rightarrow \cdots \rightarrow \mathcal{S}_{1}(-d-1) \rightarrow \mathcal{S}_{0}(-d) \rightarrow \mathcal{F} \rightarrow 0,
$$

where the $\mathcal{S}_{i}, i=0, \ldots, m$ are coherent sheaves on $X$. Assume that we have chosen hermitian structures on the sheaves $\mathcal{S}_{i}$. Using Notation 5.2 and [17, Def. 3.37, Def. 3.39] we have objects $[\bar{\mu}]$ in $\mathbf{K A}\left(\mathbb{P}_{X}^{n}\right)$ and $\left[\overline{\pi_{*} \mu}\right]$ in $\mathbf{K A}(X)$. Then we write

$$
T_{\mathfrak{t}, \bar{\mu}}(\bar{\xi})=\sum_{j=0}^{m}(-1)^{j} t_{n, j-d} \operatorname{ch}\left(\overline{\mathcal{S}}_{j}\right)-\widetilde{\operatorname{ch}}\left(\overline{\pi_{*} \mu}\right)+\bar{\pi}_{b}^{\mathrm{FS}}(\widetilde{\operatorname{ch}}(\bar{\mu}))
$$

Lemma 5.27. Given any choice of metrics on the sheaves $\mathcal{G}_{i}$, (respectively $\left.\mathcal{G}_{i}^{\prime}\right) i=$ $0, \ldots, n$, that appear in the resolution $\gamma_{d}(\mathcal{F})$ (respectively $\gamma_{d+1}(\mathcal{F})$ ), denote by $\bar{\gamma}_{d}$ and $\bar{\gamma}_{d+1}$ the corresponding exact sequences of metrized coherent sheaves. Then

$$
T_{\mathfrak{t}, \bar{\gamma}_{d+1}}(\bar{\xi})=T_{\mathfrak{t}, \bar{\gamma}_{d}}(\bar{\xi})
$$

In particular, $T_{\mathfrak{t}, \bar{\gamma}_{d}}(\bar{\xi})$ does not depend on the choice of metrics on the sheaves $\mathcal{G}_{i}$.

Proof. By Theorem 4.5(ii), there is an exact sequence

$$
\bar{\mu}: 0 \rightarrow \overline{\mathcal{S}}_{n+k}(-n-k-d-1) \rightarrow \cdots \rightarrow \overline{\mathcal{S}}_{0}(-d-1) \rightarrow \overline{\mathcal{F}} \rightarrow 0,
$$

and a surjection of exact sequences $f: \bar{\mu} \rightarrow \bar{\gamma}_{d}$ extending the identity on $\overline{\mathcal{F}}$. Here $\overline{\mathcal{S}}_{i}$, $i=0, \ldots, n+k$ are coherent sheaves on $X$ with hermitian structures.

By Theorem 4.5(i) there is a surjection of exact sequences $\bar{\mu} \longrightarrow \bar{\gamma}_{d+1}$ extending the identity on $\overline{\mathcal{F}}$, whose kernel is an exact sequence

$$
\bar{\varepsilon}: 0 \rightarrow \overline{\mathcal{M}}_{n+k}(-n-k-d-1) \rightarrow \cdots \rightarrow \overline{\mathcal{M}}_{0}(-d-1) \rightarrow 0,
$$

where $\overline{\mathcal{M}}_{i}, i=0, \ldots, n+k$ are coherent sheaves on $X$, and we have chosen arbitrarily an hermitian structure on them. Denote by $\bar{\eta}_{i}$ the rows of the exact sequence

$$
0 \rightarrow \bar{\varepsilon} \rightarrow \bar{\mu} \rightarrow \bar{\gamma}_{d+1} \rightarrow 0
$$

Observe that $\bar{\eta}_{i}=\bar{\eta}_{i}^{\prime}(-i-d-1)$ for some short exact sequence $\bar{\eta}_{i}^{\prime}$ on $X$. When $j \geq n$ we denote $\overline{\mathcal{G}}_{j}^{\prime}=\overline{0}$. Then, we have

$$
\begin{aligned}
\sum_{j=0}^{n+k}(-1)^{j} t_{n, j-d-1}\left(\operatorname{ch}\left(\overline{\mathcal{G}}_{j}^{\prime}\right)-\operatorname{ch}\left(\overline{\mathcal{S}}_{j}\right)+\operatorname{ch}\left(\overline{\mathcal{M}}_{j}\right)\right)= & \\
& \sum_{j=0}^{n+k}(-1)^{j} t_{n, j-d-1} \mathrm{~d}_{\mathcal{D}} \widetilde{c h}^{\prime}\left(\bar{\eta}_{i}^{\prime}\right)=0 .
\end{aligned}
$$


By [17, Prop. 3.41], we have

$$
\begin{aligned}
& \widetilde{\operatorname{ch}}\left(\overline{\pi_{*} \gamma_{d+1}}\right)-\widetilde{\operatorname{ch}}\left(\overline{\pi_{*} \mu}\right)+\widetilde{\operatorname{ch}}\left(\overline{\pi_{*} \varepsilon}\right)=\sum_{j=0}^{n+k}(-1)^{j} \widetilde{\operatorname{ch}}\left(\overline{\pi_{*} \eta_{j}}\right), \\
& \widetilde{\operatorname{ch}}\left(\bar{\gamma}_{d+1}\right)-\widetilde{\operatorname{ch}}(\bar{\mu})+\widetilde{\operatorname{ch}}(\bar{\varepsilon})=\sum_{j=0}^{n+k}(-1)^{j} \widetilde{\operatorname{ch}}\left(\bar{\eta}_{j}\right) .
\end{aligned}
$$

Combining equations (5.30), (5.31) and (5.32) and lemmas 5.20 and 5.23 we obtain

$$
T_{\mathfrak{t}, \bar{\mu}}(\bar{\xi})=T_{\mathfrak{t}, \bar{\gamma}_{d+1}}(\bar{\xi})
$$

We consider now cone $\left(\mu, \gamma_{d}\right)$. On it we put the obvious hermitian structure induced by $\bar{\mu}$ and $\overline{\gamma_{d}}, \overline{\operatorname{cone}\left(\mu, \gamma_{d}\right)}$. On $\pi_{*} \operatorname{cone}\left(\mu, \gamma_{d}\right)$, we put the obvious family of hermitian metrics induced by $\overline{\pi_{*} \mu}$ and $\overline{\pi_{*} \gamma_{d}}$, and denote it as $\overline{\pi_{*} \operatorname{cone}\left(\mu, \gamma_{d}\right)}$. By [17, Cor. 3.42] we have

$$
\begin{aligned}
\widetilde{\operatorname{ch}}\left(\overline{\operatorname{cone}\left(\mu, \gamma_{d}\right)}\right) & =\widetilde{\operatorname{ch}}\left(\bar{\gamma}_{d}\right)-\widetilde{\operatorname{ch}}(\bar{\mu}), \\
\widetilde{\operatorname{ch}}\left(\overline{\pi_{*} \operatorname{cone}\left(\mu, \gamma_{d}\right)}\right) & =\widetilde{\operatorname{ch}}\left(\overline{\pi_{*} \gamma_{d}}\right)-\widetilde{\operatorname{ch}}\left(\overline{\pi_{*} \mu}\right) .
\end{aligned}
$$

Observe that $\overline{\text { cone }\left(\mu, \gamma_{d}\right)}{ }^{i}=\overline{\mathcal{S}}_{-i-1}(i-d) \oplus \overline{\mathcal{G}}_{-i}(i-d)$. Combining Lemma 5.23 for $\overline{\operatorname{cone}\left(\mu, \gamma_{d}\right)}$ with equations (5.34) and (5.35), we obtain

$$
T_{\mathfrak{t}, \bar{\mu}}(\bar{\xi})=T_{\mathfrak{t}, \bar{\gamma}_{d}}(\bar{\xi})
$$

Together with equation (5.33) this proves the lemma.

Now we are in position to prove the existence of $T_{\mathfrak{t}}$. Let $n$ and $\mathfrak{t}$ be as in Theorem 5.9. We define the numbers $t_{n, k}$, for $k<0$ and $k>n$ by equation (5.19). Let $\bar{\xi}=\left(\bar{\pi}^{\mathrm{FS}}, \overline{\mathcal{F}}, \overline{\pi_{*} \mathcal{F}}\right)$ be a relative metrized complex. We construct $T_{\mathfrak{t}}(\bar{\xi})$ by induction on the length of the cohomology of $\mathcal{F}$. If it has at most a single non zero coherent sheaf $\mathcal{H}$ sitting at degree $j$, then $\overline{\mathcal{F}}$ and $\overline{\pi_{*} \mathcal{F}}$ determine hermitian structures on $\mathcal{H}[-j]$ and $\pi_{*} \mathcal{H}[-j]$ respectively. We choose an integer $d$ such that $\mathcal{H}(d)$ is regular and we write

$$
T_{\mathfrak{t}}(\bar{\xi})=(-1)^{j} T_{\mathfrak{t}, \bar{\gamma}_{d}(\mathcal{H})}\left(\bar{\pi}^{\mathrm{FS}}, \overline{\mathcal{H}}, \overline{\pi_{*} \mathcal{H}}\right) .
$$

By Lemma 5.27, this does not depend on the choice of $d$ nor on the choice of metrics on $\bar{\gamma}_{d}(\mathcal{H})$.

Assume that we have defined the analytic torsion classes for all complexes whose cohomology has length less than $l$ and that the cohomology of $\mathcal{F}$ has length $l$. Let $\mathcal{H}$ be the highest cohomology sheaf of $\mathcal{F}$, say of degree $j$. Choose auxiliary hermitian structures on $\mathcal{H}[-j]$ and $\pi_{*} \mathcal{H}[-j]$. There is a unique natural map $\mathcal{H}[-j] \rightarrow \mathcal{F}$. Then we define

$$
\begin{aligned}
T_{\mathfrak{t}}(\bar{\xi})=T_{\mathfrak{t}}\left(\bar{\pi}^{\mathrm{FS}}, \overline{\mathcal{H}[-j]}, \overline{\pi_{*} \mathcal{H}[-j]}\right) & \\
& +T_{\mathfrak{t}}\left(\bar{\pi}^{\mathrm{FS}}, \overline{\operatorname{cone}}(\overline{\mathcal{H}[-j]}, \overline{\mathcal{F}}), \overline{\operatorname{cone}}\left(\overline{\pi_{*} \mathcal{H}[-j]}, \overline{\pi_{*} \mathcal{F}}\right)\right) .
\end{aligned}
$$

It follows from [17, Thm. 2.27 (iv)] that the right hand side of this equality does not depend on the choice of the auxiliary hermitian structures. 
Finally, we consider the case when $\bar{\pi}$ has a metric different from the Fubini-Study metric. Thus, let $\bar{\xi}=\left(\bar{\pi}, \overline{\mathcal{F}}, \overline{\pi_{*} \mathcal{F}}\right)$ and write $\bar{\xi}^{\prime}=\left(\bar{\pi}^{\mathrm{FS}}, \overline{\mathcal{F}}, \overline{\pi_{*} \mathcal{F}}\right)$. Then we put

$$
T_{\mathfrak{t}}(\bar{\xi})=T_{\mathfrak{t}}\left(\bar{\xi}^{\prime}\right)+\bar{\pi}_{b}\left[\operatorname{ch}(\bar{F}) \bullet \widetilde{\mathrm{Td}}_{m}\left(\bar{\pi}, \bar{\pi}^{\mathrm{FS}}\right)\right] .
$$

Definition 5.40. Let $n$ and $\mathfrak{t}$ be as in Theorem 5.9. Then $T_{\mathfrak{t}}$ is the assignment that to each relative metrized complex $\bar{\xi}$ associates $T_{\mathfrak{t}}(\bar{\xi})$ given by equations (5.37), (5.38) and (5.39).

It remains to prove that $T_{\mathfrak{t}}$ satisfies axioms (i) to (iv). Axiom (i) follows from the differential equations satisfied by the Bott-Chern classes. Axiom (ii) follows from the functoriality of the canonical resolution, the Chern forms and the Bott-Chern classes. Axiom (iii) follows from the additivity of the canonical resolution and of the Chern character. Finally Axiom (iv) follows from the multiplicativity of the Chern character. This concludes the proof of Theorem 5.9 .

We finish this section showing the compatibility of analytic torsion classes with the composition of projective bundles. Let $X$ be a smooth complex variety. Consider the commutative diagram with cartesian square

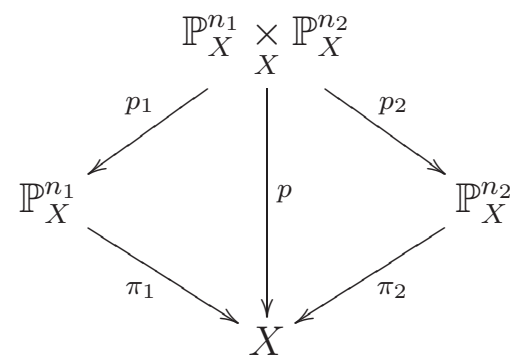

On $\pi_{1}$ and $\pi_{2}$ we introduce arbitrary hermitian structures and on $p_{1}$ and $p_{2}$ the hermitian structures induced by the cartesian diagram.

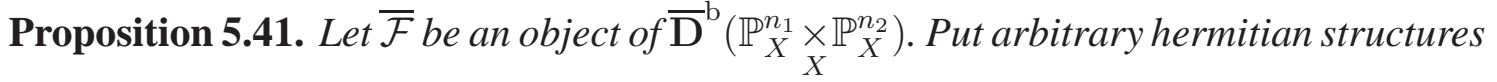
on $\left(p_{1}\right)_{*} \mathcal{F},\left(p_{2}\right)_{*} \mathcal{F}$, and $p_{*} \mathcal{F}$. Then

$$
T\left(\bar{\pi}_{1}\right)+\left(\bar{\pi}_{1}\right)_{b}\left(T\left(\bar{p}_{1}\right)\right)=T\left(\bar{\pi}_{2}\right)+\left(\bar{\pi}_{2}\right)_{b}\left(T\left(\bar{p}_{2}\right)\right),
$$

where we are using the convention at the end of Definition 2.6

Proof. By the anomaly formulas (Proposition 5.10), if equation (5.42) holds for a particular choice of hermitian structures on $\mathcal{F},\left(p_{1}\right)_{*} \mathcal{F},\left(p_{2}\right)_{*} \mathcal{F}$, and $p_{*} \mathcal{F}$, then it holds for any other choice. Let

$$
\overline{\mathcal{F}}_{2}-\rightarrow \overline{\mathcal{F}}_{1--\rightarrow} \overline{\mathcal{F}}_{0--\rightarrow}
$$

be a distinguished triangle and put hermitian structures on the direct images as before. Then Proposition 5.12 implies that, if equation (5.42) holds for two of them, then it also holds for the third. Since the objects of the form $\mathcal{G}(k, l):=p^{*} \mathcal{G} \otimes p_{1}^{*} \mathcal{O}(k) \otimes p_{2}^{*} \mathcal{O}(l)$ are a generating class of $\mathbf{D}^{\mathrm{b}}\left(\mathbb{P}_{X}^{n_{1}} \times \mathbb{P}_{X}^{n_{2}}\right)$, the previous discussion shows that it is enough to prove the case $\mathcal{F}=\mathcal{G}(k, l)$, with the hermitian structure of $\mathcal{F}$ induced by a hermitian structure of $\mathcal{G}$ and the Fubini-Study metric on $\mathcal{O}(k)$ and $\mathcal{O}(l)$, and the hermitian structures on the direct images defined as in (5.3). In this case the result follows easily from the functoriality and the projection formula. 


\section{Compatible analytic torsion classes}

In this section we study the compatibility between analytic torsion classes for closed immersions and analytic torsion classes for projective spaces. It turns out that, once the compatibility between the diagonal embedding of $\mathbb{P}^{n}$ into $\mathbb{P}^{n} \times \mathbb{P}^{n}$ and the second projection of $\mathbb{P}^{n} \times \mathbb{P}^{n}$ onto $\mathbb{P}^{n}$ is established, then all the other possible compatibilities follow. Essentially this observation can be traced back to [15].

Let $n, V, \bar{V}$ and $\mathbb{P}^{n}(V)$ be as in the previous section. We consider the diagram

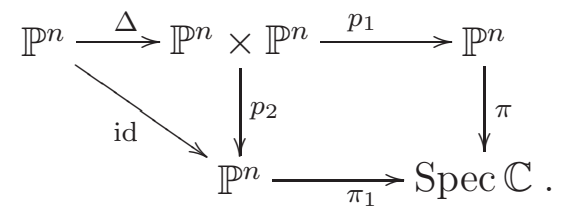

On $\mathbb{P}^{n}$ we have the tautological short exact sequence

$$
0 \rightarrow \mathcal{O}(-1) \rightarrow V \rightarrow Q \rightarrow 0
$$

This induces on $\mathbb{P}^{n} \times \mathbb{P}^{n}$ the exact sequence

$$
0 \rightarrow p_{2}^{*} \mathcal{O}(-1) \rightarrow V \rightarrow p_{2}^{*} Q \rightarrow 0
$$

By composition with the injection $p_{1}^{*} \mathcal{O}(-1) \hookrightarrow V$, we obtain a morphism $p_{1}^{*} \mathcal{O}(-1) \rightarrow$ $p_{2}^{*} Q$, hence a section of $p_{2}^{*} Q \otimes p_{1}^{*} \mathcal{O}(1)$. The zero locus of this section is the image of the diagonal. Moreover, the associated Koszul complex is quasi-isomorphic to $\Delta_{*} \mathcal{O}_{\mathbb{P} n}$. That is, the sequence

$$
\begin{aligned}
0 \rightarrow \Lambda^{n}\left(p_{2}^{*} Q^{\vee}\right) \otimes p_{1}^{*} & \mathcal{O}_{\mathbb{P}^{n}}(-n) \rightarrow \ldots \\
\cdots & \rightarrow \Lambda^{1}\left(p_{2}^{*} Q^{\vee}\right) \otimes p_{1}^{*} \mathcal{O}_{\mathbb{P}^{n}}(-1) \rightarrow \mathcal{O}_{\mathbb{P}^{n} \times \mathbb{P}^{n}} \rightarrow \Delta_{*} \mathcal{O}_{\mathbb{P}^{n}} \rightarrow 0
\end{aligned}
$$

is exact.

On $T_{\mathbb{P}^{n}}$ and $T_{\mathbb{P}^{n} \times \mathbb{P}^{n}}$ we consider the Fubini-Study metrics. We denote by $\bar{\Delta}$ and $\bar{p}_{2}$ the morphisms of $\overline{\mathbf{S m}}_{* / \mathbb{C}}$ determined by these metrics. As in [17, Ex. 5.7], we have that $\overline{p_{2}} \circ \bar{\Delta}=\overline{\mathrm{id}}_{\mathbb{P}^{n}}$, where $T_{\overline{\mathrm{id} p} n}=\overline{0}$. The Fubini-Study metric on $\mathcal{O}(-1)$ and the metric induced by the tautological exact sequence on $Q$ induce a metric $\bar{K}(\Delta)$ on the Koszul complex. This is a hermitian structure on $\Delta_{*} \mathcal{O}_{\mathbb{P}^{n}}$. Finally on $\mathcal{O}_{\mathbb{P}^{n}}$ we consider the trivial metric. This is a hermitian structure on $\left(p_{2}\right)_{*} K(\Delta)$.

Fix a real additive genus $S$ and denote by $T_{S}$ the theory of analytic torsion classes for closed immersions that is compatible with the projection formula and transitive, associated to $S$ (Theorem 3.11). Moreover, fix a family of real numbers $\mathfrak{t}=\left\{t_{n k} \mid n \geq\right.$ $0,-n \leq k \leq 0\}$ and denote $T_{\mathfrak{t}}$ the theory of generalized analytic torsion classes for projective spaces associated to this family.

Compatible analytic torsion classes for closed immersions and for projective spaces should combine to provide analytic torsion classes for arbitrary projective morphisms, and these classes should be transitive. The transitivity condition for the composition $\mathrm{id}_{\mathbb{P}^{n}}=$ $p_{2} \circ \Delta$ should give us

$$
0=T\left(\overline{\mathrm{id}}_{\mathbb{P}^{n}}, \overline{\mathcal{O}}_{\mathbb{P}^{n}}, \overline{\mathcal{O}}_{\mathbb{P}^{n}}\right)=T_{\mathfrak{t}}\left(\overline{p_{2}}, \bar{K}(\Delta), \overline{\mathcal{O}}_{\mathbb{P}^{n}}\right)+\left(\bar{p}_{2}\right)_{b}\left(T_{S}\left(\bar{\Delta}, \overline{\mathcal{O}}_{\mathbb{P}^{n}}, \bar{K}(\Delta)\right)\right) .
$$

In general we define 
Definition 6.2. The theories of analytic torsion classes $T_{S}$ and $T_{\mathfrak{t}}$ are called compatible if

$$
T_{\mathfrak{t}}\left(\overline{p_{2}}, \bar{K}(\Delta), \overline{\mathcal{O}}_{\mathbb{P}^{n}}\right)+\left(\bar{p}_{2}\right)_{b}\left(T_{S}\left(\bar{\Delta}, \overline{\mathcal{O}}_{\mathbb{P}^{n}}, \bar{K}(\Delta)\right)\right)=0 .
$$

Theorem 6.4. Let $S$ be a real additive genus. Then there exists a unique family of real numbers $\mathfrak{t}=\left\{t_{n, k} \mid n \geq 0,-n \leq k \leq 0\right\}$ such that the theories of analytic torsion classes $T_{S}$ and $T_{\mathfrak{t}}$ are compatible. The theory $T_{\mathfrak{t}}$ will also be denoted $T_{S}$.

Proof. The first step is to make explicit equation (6.3) in terms of the main characteristic numbers $\mathfrak{t}$. To this end, first observe that, since the exact sequence

$$
0 \rightarrow T_{p_{2}} \rightarrow T_{\mathbb{P}^{n} \times \mathbb{P}^{n}} \rightarrow p_{2}^{*} T_{\mathbb{P}^{n}} \rightarrow 0
$$

is split and the hermitian metric on $T_{\mathbb{P}^{n} \times \mathbb{P}^{n}}$ is the orthogonal direct sum metric, $\overline{p_{2}}=$ $\pi_{1}^{*}\left(\bar{\pi}^{\mathrm{FS}}\right)$. Next, we denote by $\bar{K}(\Delta)_{i}$ the component of degree $i$ of the Koszul complex, and we define

$$
\overline{\left(p_{2}\right)_{*} K(\Delta)_{i}}= \begin{cases}\overline{\mathcal{O}}_{\mathbb{P}^{n}}, & \text { for } i=0 \\ \overline{0}, & \text { for } i>0\end{cases}
$$

Finally using Corollary 5.15, functoriality and the compatibility with the projection formula, we derive

$$
\begin{aligned}
T_{\mathfrak{t}}\left(\overline{p_{2}}, \bar{K}(\Delta), \overline{\mathcal{O}}_{\mathbb{P} n}\right) & =\sum_{i=0}^{n}(-1)^{i} T_{\mathfrak{t}}\left(\overline{p_{2}}, \bar{K}(\Delta)_{i}, \overline{\left(p_{2}\right)_{*} K(\Delta)_{i}}\right) \\
& =\sum_{i=0}^{n}(-1)^{i} T_{\mathfrak{t}}\left(\pi_{1}^{*} \bar{\xi}_{n}(-i) \otimes \Lambda^{i} \bar{Q}^{\vee}\right) \\
& =\sum_{i=0}^{n}(-1)^{i} t_{n,-i} \operatorname{ch}\left(\Lambda^{i} \bar{Q}^{\vee}\right) .
\end{aligned}
$$

Thus, the second and last step is to solve the equation

$$
\sum_{i=0}^{n}(-1)^{i} t_{n,-i} \operatorname{ch}\left(\Lambda^{i} \bar{Q}^{\vee}\right)=-\left(p_{2}\right)_{*}\left(T_{S}\left(\bar{\Delta}, \overline{\mathcal{O}}_{\mathbb{P}^{n}}, \bar{K}(\Delta)\right) \bullet \operatorname{Td}\left(\overline{p_{2}}\right)\right) .
$$

Since the left hand side of equation (6.6) is closed, in order to be able to solve this equation we have to show that the right hand side is also closed. We compute

$$
\begin{aligned}
\mathrm{d}_{\mathcal{D}}\left(p_{2}\right)_{*} & \left(T_{S}\left(\bar{\Delta}, \overline{\mathcal{O}}_{\mathbb{P}^{n}}, \bar{K}(\Delta)\right) \bullet \operatorname{Td}\left(\bar{p}_{2}\right)\right) \\
= & \left(p_{2}\right)_{*}\left(\sum_{i=0}^{n}(-1)^{i} \operatorname{ch}\left(\bar{K}(\Delta)_{i}\right) \operatorname{Td}\left(\bar{p}_{2}\right)-\Delta_{*}\left(\operatorname{ch}\left(\overline{\mathcal{O}}_{\mathbb{P}^{n}}\right) \operatorname{Td}(\bar{\Delta})\right) \operatorname{Td}\left(\bar{p}_{2}\right)\right) \\
= & \left(p_{2}\right)_{*}\left(\sum_{i=0}^{n}(-1)^{i} p_{2}^{*}\left(\operatorname{ch}\left(\Lambda^{i} \bar{Q}^{\vee}\right)\right) p_{1}^{*}(\operatorname{ch}(\overline{\mathcal{O}}(-i))) \operatorname{Td}\left(\bar{p}_{2}\right)\right)-1 \\
& =\sum_{i=0}^{n}(-1)^{i} \operatorname{ch}\left(\Lambda^{i} \bar{Q}^{\vee}\right)\left(p_{2}\right)_{*}\left(p_{1}^{*}(\operatorname{ch}(\overline{\mathcal{O}}(-i))) \operatorname{Td}\left(\bar{p}_{2}\right)\right)-1 \\
& =\sum_{i=0}^{n}(-1)^{i} \operatorname{ch}\left(\Lambda^{i} \bar{Q}^{\vee}\right) \pi_{1}^{*} \pi_{*}(\operatorname{ch}(\overline{\mathcal{O}}(-i)) \operatorname{Td}(\bar{\pi}))-1 \\
& =1-1=0 .
\end{aligned}
$$


In the first equality we have used the differential equation of $T_{S}$. In the second one we have used the definition of the Koszul complex, the equation $\operatorname{ch}\left(\overline{\mathcal{O}}_{\mathbb{P} n}\right)=1$ and the fact that, by the choice of hermitian structures on $T_{\bar{\Delta}}$ and $T_{\bar{p}_{2}}$ we have $\operatorname{Td}(\bar{\Delta}) \bullet \Delta^{*}\left(\operatorname{Td}\left(\bar{p}_{2}\right)\right)=1$. The third equality is the projection formula and the fourth is base change for cohomology. For the last equality we have used equation (5.22).

Both sides of equation (6.6) are closed and defined up to boundaries, hence this is an equation in cohomology classes. The tautological exact sequence induces exact sequences

$$
0 \rightarrow \Lambda^{k} Q^{\vee} \rightarrow \Lambda^{k} V^{\vee} \rightarrow \Lambda^{k-1} Q^{\vee} \otimes \mathcal{O}(1) \rightarrow 0
$$

that give us equations

$$
\operatorname{ch}\left(\Lambda^{k} Q^{\vee}\right)=\left(\begin{array}{c}
n+1 \\
k
\end{array}\right)-\operatorname{ch}\left(\Lambda^{k-1} Q^{\vee}\right) \operatorname{ch}(\mathcal{O}(1)) .
$$

Hence

$$
\operatorname{ch}\left(\Lambda^{k} Q^{\vee}\right)=\sum_{i=0}^{k}(-1)^{i}\left(\begin{array}{c}
n+1 \\
k-i
\end{array}\right) \operatorname{ch}(\mathcal{O}(i)) .
$$

Since the classes $\operatorname{ch}(\mathcal{O}(i)), i=0, \ldots, n$, form a basis of $\bigoplus_{p} H_{\mathcal{D}}^{2 p}\left(\mathbb{P}^{n}, \mathbb{R}(p)\right)$, the same is true for the classes $\operatorname{ch}\left(\Lambda^{i} Q^{\vee}\right), i=0, \ldots, n$. Therefore, if $\mathbf{1}_{1} \in H_{\mathcal{D}}^{1}\left(\mathbb{P}^{n}, \mathbb{R}(1)\right)$ is the class represented by the constant function 1 , the classes $\mathbf{1}_{1} \bullet \operatorname{ch}\left(\Lambda^{i} Q^{\vee}\right), i=0, \ldots, n$ form a basis of $\bigoplus_{p=1}^{n+1} H_{\mathcal{D}}^{2 p-1}\left(\mathbb{P}^{n}, \mathbb{R}(p)\right)$. This implies that equation (6.6) has a unique solution.

Remark 6.7. Given a theory $T$ of analytic torsion classes for projective spaces, obtained from an arbitrary choice of characteristic numbers, in general, it does not exist an additive genus such that the associated theory of singular Bott-Chern classes is compatible with $T$. It would be interesting to characterize the collections of characteristic numbers that arise from Theorem 6.4.

By definition, compatible analytic torsion classes for closed immersions and projective spaces satisfy a compatibility condition for the trivial vector bundle and the diagonal embedding. When adding the functoriality and the projection formula, we obtain compatibility relations for arbitrary sections of the trivial projective bundle and arbitrary objects.

Let $X$ be a smooth complex variety, let $\pi: \mathbb{P}_{X}^{n} \rightarrow X$ be the projective space over $X$ and let $s: X \rightarrow \mathbb{P}_{X}^{n}$ be a section. Choose any hermitian structure on $T_{\pi}$. Since we have an isomorphism $T_{s} \rightarrow s^{*} T_{\pi}[-1]$, this hermitian structure induces a hermitian structure on $s$. Denote by $\bar{\pi}$ and $\bar{s}$ the corresponding morphisms in $\overline{\mathbf{S m}}_{* / \mathbb{C}}$. With this choice of hermitian structures, we have

$$
\bar{\pi} \circ \bar{s}=\left(\pi \circ s, \overline{\operatorname{cone}}\left(s^{*} T_{\bar{\pi}}[-1], s^{*} T_{\bar{\pi}}[-1]\right)\right)=\left(\operatorname{id}_{X}, \overline{0}\right),
$$

because the cone of the identity is meager.

Proposition 6.8. Let $S$ be a real additive genus. Let $T_{S}$ denote both, the theory of analytic torsion classes for closed immersions determined by $S$, and the theory of analytic torsion classes for projective spaces compatible with it. Let $\overline{\mathcal{F}}$ be an object of $\overline{\mathbf{D}}^{\mathrm{b}}(X)$. Put a hermitian structure on $s_{*} \mathcal{F}$. Then

$$
T_{S}\left(\bar{\pi}, \overline{s_{*} \mathcal{F}}, \overline{\mathcal{F}}\right)+\bar{\pi}_{b}\left(T_{S}\left(\bar{s}, \overline{\mathcal{F}}, \overline{s_{*} \mathcal{F}}\right)\right)=0 .
$$


Proof. By the anomaly formulas Proposition 3.17 and Proposition 5.10, if equation (6.9) holds for a particular choice of hermitian structure on $s_{*} \mathcal{F}$ then it holds for any other choice. Therefore we can assume that the hermitian structure on $s_{*} \mathcal{F}$ is given by $\bar{K}(s) \otimes$ $\pi^{*} \overline{\mathcal{F}}$, where $\bar{K}(s)$ is the Koszul complex associated to the section $s$. By the projection formulas, if (6.9) holds for the trivial bundle $\mathcal{O}_{X}$ then it holds for arbitrary objects of $\overline{\mathbf{D}}^{\mathrm{b}}(X)$.

We now prove that, if equation (6.9) holds for a particular choice of hermitian structure $\bar{\pi}$, then it holds for any other choice. Thus, assume that equation (6.9) is satisfied for $\bar{\pi}$ and $\bar{s}$. Let $\bar{\pi}^{\prime}$ be another choice of hermitian structure on $\pi$ and $\bar{s}^{\prime}$ be the hermitian structure induced on $s$. On one hand, we have

$$
T_{S}\left(\bar{\pi}^{\prime}, \bar{K}(s), \overline{\mathcal{O}}_{X}\right)=T_{S}\left(\bar{\pi}, \bar{K}(s), \overline{\mathcal{O}}_{X}\right)+\pi_{*}\left(\operatorname{ch}\left(\bar{K}(s) \bullet \widetilde{\mathrm{Td}}_{m}\left(\bar{\pi}^{\prime}, \bar{\pi}\right) \bullet \operatorname{Td}\left(\bar{\pi}^{\prime}\right)\right)\right) .
$$

On the other hand, we have

$$
\begin{aligned}
& T_{S}\left(\bar{s}^{\prime}, \overline{\mathcal{F}}, \overline{s_{*} \mathcal{F}}\right) \bullet \operatorname{Td}\left(\bar{\pi}^{\prime}\right) \\
& =\left(T_{S}\left(\bar{s}, \overline{\mathcal{F}}, \overline{s_{*} \mathcal{F}}\right)+s_{*}\left(\widetilde{\operatorname{Td}}_{m}\left(\bar{s}^{\prime}, \bar{s}\right) \operatorname{Td}\left(\bar{s}^{\prime}\right)\right)\right) \bullet\left(\operatorname{Td}(\bar{\pi})-\mathrm{d}_{\mathcal{D}}\left(\widetilde{\operatorname{Td}}_{m}\left(\bar{\pi}^{\prime}, \bar{\pi}\right) \bullet \operatorname{Td}\left(\bar{\pi}^{\prime}\right)\right)\right) \\
& =T_{S}\left(\bar{s}, \overline{\mathcal{F}}, \overline{s_{*} \mathcal{F}}\right) \bullet \operatorname{Td}(\bar{\pi})+s_{*}\left(\widetilde{\operatorname{Td}}{ }_{m}\left(\bar{s}^{\prime}, \bar{s}\right) \operatorname{Td}\left(\bar{s}^{\prime}\right)\right) \bullet \operatorname{Td}\left(\bar{\pi}^{\prime}\right) \\
& \quad-T_{S}\left(\bar{s}, \overline{\mathcal{F}}, \overline{s_{*} \mathcal{F}}\right) \bullet \mathrm{d}_{\mathcal{D}}\left(\widetilde{\operatorname{Td}}_{m}\left(\bar{\pi}^{\prime}, \bar{\pi}\right) \bullet \operatorname{Td}\left(\bar{\pi}^{\prime}\right)\right)
\end{aligned}
$$

In the group $\bigoplus_{p} \widetilde{D}_{D}^{2 p-1}\left(\mathbb{P}_{X}^{n}, N_{s}, p\right)$ we have

$$
\begin{aligned}
T_{S}\left(\bar{s}, \overline{\mathcal{F}}, \overline{s_{*} \mathcal{F}}\right) \bullet \mathrm{d}_{\mathcal{D}}\left(\widetilde{\mathrm{Td}}_{m}\left(\bar{\pi}^{\prime}, \bar{\pi}\right) \bullet \operatorname{Td}\left(\bar{\pi}^{\prime}\right)\right) \\
\quad=\left(\operatorname{ch}(\bar{K}(s))-s_{*}(\operatorname{Td}(\bar{s}))\right) \bullet\left(\widetilde{\operatorname{Td}}_{m}\left(\bar{\pi}^{\prime}, \bar{\pi}\right) \bullet \operatorname{Td}\left(\bar{\pi}^{\prime}\right)\right) .
\end{aligned}
$$

Observe that, by the definition of the hermitian structure of $\bar{s}$ and $\bar{s}^{\prime}$ we have

$$
\operatorname{Td}(\bar{s}) \bullet s^{*} \widetilde{\operatorname{Td}}_{m}\left(\bar{\pi}^{\prime}, \bar{\pi}\right)=-\widetilde{\mathrm{Td}}_{m}\left(\bar{s}^{\prime}, \bar{s}\right) \bullet \operatorname{Td}\left(\bar{s}^{\prime}\right) \text {. }
$$

By combining equations (6.9), (6.10), 6.12) and (6.13) we obtain

$$
T_{S}\left(\bar{\pi}^{\prime}, \overline{s_{*} \mathcal{F}}, \overline{\mathcal{F}}\right)=-\pi_{*}\left(T_{S}\left(\bar{s}^{\prime}, \overline{\mathcal{F}}, \overline{s_{*} \mathcal{F}}\right) \bullet \operatorname{Td}\left(\bar{\pi}^{\prime}\right)\right) .
$$

We now prove (6.9) for a particular choice of hermitian structures. Let $f: X \rightarrow \mathbb{P}^{n}$ denote the composition of $s$ with the projection $\mathbb{P}_{X}^{n} \rightarrow \mathbb{P}^{n}$. Then we have a commutative diagram with cartesian squares

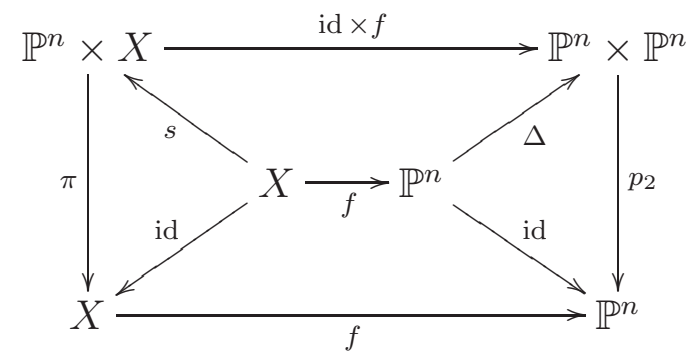

Let $\bar{\Delta}$ and $\bar{p}_{2}$ be as in Definition 6.2. On $\bar{\pi}$ and $\bar{s}$ we put the hermitian structures induced by $\bar{\Delta}$. Since the Koszul complex $\bar{K}(s)=\left(\operatorname{id}_{\mathbb{P}^{n}} \times f\right)^{*} \bar{K}(\Delta)$, by Proposition 2.15 and functoriality, equation 6.9) in this case follows from equation (6.3). 
We now study another compatibility between analytic torsion classes for closed immersions and projective spaces. Let $\iota: X \rightarrow Y$ be a closed immersion of smooth complex varieties. Consider the cartesian square

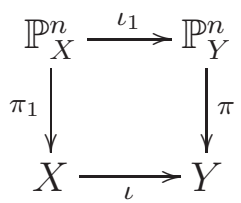

Choose hermitian structures on $\pi$ and $\iota$ and put on $\pi_{1}$ and $\iota_{i}$ the induced ones.

Proposition 6.15. Let $S$ be a real additive genus. Let $T_{S}$ denote both, the theory of analytic torsion classes for closed immersions determined by $S$, and the theory of analytic torsion classes for projective spaces compatible with it. Let $\overline{\mathcal{F}}$ be and object of $\overline{\mathbf{D}}^{\mathrm{b}}\left(\mathbb{P}_{X}^{n}\right)$. Put hermitian structures on $\left(\pi_{1}\right)_{*} \mathcal{F},\left(\iota_{1}\right)_{*} \mathcal{F}$ and $\left(\pi \circ \iota_{1}\right)_{*} \mathcal{F}$. Then

$$
T_{S}(\bar{\pi})+\bar{\pi}_{b}\left(T_{S}\left(\overline{\iota_{1}}\right)\right)=T_{S}(\bar{\iota})+\bar{\iota}_{b}\left(T_{S}\left(\overline{\pi_{1}}\right)\right) .
$$

Proof. By the anomaly formulas, if equation (6.16) holds for a particular choice of metrics on $\left(\pi_{1}\right)_{*} \mathcal{F},\left(\iota_{1}\right)_{*} \mathcal{F}$ and $\left(\pi \circ \iota_{1}\right)_{*} \mathcal{F}$, then it holds for any choice. Because the sheaves $\mathcal{G}(k)$, with $\mathcal{G}$ a coherent sheaf on $X$, constitute a generating class of $\mathbf{D}^{\mathrm{b}}\left(\mathbb{P}_{X}^{n}\right)$ and by propositions 3.19 and 5.12, we reduce to the case when $\mathcal{F}$ is of the form $\mathcal{G}(k)$. We choose arbitrary hermitian structures on $\mathcal{G}$ and $\iota_{*} \mathcal{G}$. Furthermore, we assume $\mathcal{O}(k),\left(\pi_{1}\right)_{*} \mathcal{O}(k)$ and $\pi_{*} \mathcal{O}(k)$ endowed with the hermitian structures of Notation 5.2. From these choices and the projection formula, the objects $\left(\pi_{1}\right)_{*} \mathcal{F},\left(\iota_{1}\right)_{*} \mathcal{F}$ and $\left(\pi \circ \iota_{1}\right)_{*} \mathcal{F}$ automatically inherit hermitian structures. Indeed, it is enough to observe the natural isomorphisms

$$
\begin{aligned}
& \left(\pi_{1}\right)_{*} \mathcal{F} \cong \mathcal{G} \otimes\left(\pi_{1}\right)_{*} \mathcal{O}(k) \\
& \left(\iota_{1}\right)_{*}\left(\pi_{1}^{*} \mathcal{G} \otimes \iota_{1}^{*} \mathcal{O}(k)\right) \cong \pi^{*}\left(\iota_{*} \mathcal{G}\right) \otimes \mathcal{O}(k) \\
& \left(\pi \circ \iota_{1}\right)_{*} \mathcal{F} \cong \pi_{*}\left(\pi^{*} \iota_{*} \mathcal{G} \otimes \mathcal{O}(k)\right) \cong \iota_{*} \mathcal{G} \otimes \pi_{*} \mathcal{O}(k) .
\end{aligned}
$$

We now work out the left hand side of equation (6.16). Using the projection formula for the theory $T_{S}$ for projective spaces, and equations (6.17)-6.19), we find

$$
T_{S}(\bar{\pi})=t_{n, k} \bullet \operatorname{ch}\left(\overline{\iota_{*} \mathcal{G}}\right) .
$$

Using the functoriality of $T_{S}$ for closed immersions and the projection formula we have

$$
\begin{aligned}
T_{S}\left(\bar{\iota}_{1}\right) & =\pi^{*} T_{S}\left(\iota, \overline{\mathcal{G}}, \overline{\iota_{*} \mathcal{G}}\right) \bullet \operatorname{ch}(\overline{\mathcal{O}(k)}) \\
\bar{\pi}_{b}\left(T_{S}\left(\bar{\iota}_{1}\right)\right) & =T_{S}\left(\iota, \overline{\mathcal{G}}, \overline{\iota_{*} \mathcal{G}}\right) \bullet \pi_{*}(\operatorname{ch}(\overline{\mathcal{O}(k)}) \bullet \operatorname{Td}(\bar{\pi})) .
\end{aligned}
$$

Now for the right hand side of 6.16). The projection formula for $T_{S}$ for closed immersions implies

$$
T_{S}(\bar{\iota})=T_{S}(\iota, \overline{\mathcal{G}}, \overline{\iota \mathcal{G}}) \bullet \operatorname{ch}\left(\overline{\pi_{*} \mathcal{O}(k)}\right) .
$$

Similarly, we obtain $T_{S}\left(\bar{\pi}_{1}\right)=t_{n, k} \bullet \operatorname{ch}(\overline{\mathcal{G}})$, and hence

$$
\bar{\iota}_{b}\left(T_{S}\left(\bar{\pi}_{1}\right)\right)=t_{n, k} \bullet \iota_{*}(\operatorname{ch}(\overline{\mathcal{G}}) \bullet \operatorname{Td}(\bar{\iota})) .
$$

Using (6.20)-(6.23), the difference of the two sides of (6.16) becomes

$$
t_{n, k} \bullet \mathrm{d}_{\mathcal{D}} T_{S}\left(\iota, \overline{\mathcal{G}}, \overline{\iota_{*} \mathcal{G}}\right)-T_{S}\left(\iota, \overline{\mathcal{G}}, \overline{\iota_{*} \mathcal{G}}\right) \bullet \mathrm{d}_{\mathcal{D}} t_{n, k}=-\mathrm{d}_{\mathcal{D}}\left(t_{n, k} \bullet T_{S}\left(\iota, \overline{\mathcal{G}}, \overline{\iota_{*} \mathcal{G}}\right)\right)=0
$$

in the group $\oplus_{p} \widetilde{\mathcal{D}}_{D}^{2 p-1}\left(Y, N_{\iota}, p\right)$. 


\section{Generalized analytic torsion classes}

In this section we will extend the definition of analytic torsion classes to arbitrary morphisms of smooth complex varieties. Our construction is based on the construction of analytic torsion classes by Zha in [50].

Definition 7.1. A theory of generalized analytic torsion classes is an assignment that, to each morphism $\bar{f}: X \rightarrow Y$ in $\overline{\mathbf{S m}}_{* / \mathbb{C}}$ and relative metrized complex $\bar{\xi}=\left(\bar{f}, \overline{\mathcal{F}}, \overline{f_{*} \mathcal{F}}\right)$, assigns a class of currents

$$
T(\bar{\xi}) \in \bigoplus_{p=1}^{n+1} \widetilde{\mathcal{D}}_{D}^{2 p-1}\left(Y, N_{f}, p\right)
$$

satisfying the following properties:

(i) (Differential equation) For any current $\eta \in T(\bar{\xi})$, we have

$$
\mathrm{d}_{\mathcal{D}} \eta=\operatorname{ch}\left(\overline{f_{*} \mathcal{F}}\right)-\bar{f}_{b}[\operatorname{ch}(\overline{\mathcal{F}})] .
$$

(ii) (Functoriality) If $g: Y^{\prime} \rightarrow Y$ is a morphism transverse to $f$, then

$$
g^{*} T(\bar{\xi})=T\left(g^{*} \bar{\xi}\right) .
$$

(iii) (Additivity and normalization) If $\bar{\xi}_{1}, \bar{\xi}_{2}$ are relative metrized complexes on $X$, then

$$
T\left(\bar{\xi}_{1} \oplus \bar{\xi}_{2}\right)=T\left(\bar{\xi}_{1}\right)+T\left(\bar{\xi}_{2}\right) \text {. }
$$

(iv) (Projection formula) If $\bar{\xi}$ is a relative metrized complex, and $\overline{\mathcal{G}} \in \mathrm{Ob} \overline{\mathbf{D}}^{\mathrm{b}}(Y)$, then

$$
T(\bar{\xi} \otimes \overline{\mathcal{G}})=T(\bar{\xi}) \bullet \operatorname{ch}(\overline{\mathcal{G}}) .
$$

(v) (Transitivity) If $\bar{f}: X \rightarrow Y, \bar{g}: Y \rightarrow Z$ are morphisms in $\overline{\mathbf{S m}}_{* / \mathbb{C}}$, and $\left(\bar{f}, \overline{\mathcal{F}}, \bar{f}_{*} \mathcal{F}\right)$ and $\left(\bar{g}, \overline{f_{*} \mathcal{F}}, \overline{(g \circ f)_{*} \mathcal{F}}\right)$ are relative metrized complexes, then

$$
T(\bar{g} \circ \bar{f})=T(\bar{g})+\bar{g}_{b}(T(\bar{f})) .
$$

Propositions 7.4 and 7.6 below contain several anomaly and compatibility formulas satisfied by an arbitrary theory of generalized analytic torsion classes. They follow from properties (i) (iii) and are analogous to those in propositions 3.17 and 5.10, 3.19 and 5.12 respectively. The proofs are omitted, as they are similar to those of the analogous statements referred to hereinbefore.

Proposition 7.4. Let $T$ be a theory of generalized analytic torsion classes. Let $\bar{\xi}=$ $\left(\bar{f}, \overline{\mathcal{F}}, \overline{f_{*} \mathcal{F}}\right)$ be a relative metrized complex.

(i) If $\overline{\mathcal{F}}^{\prime}$ is another choice of metric on $\mathcal{F}$ and $\bar{\xi}_{1}=\left(\bar{f}, \overline{\mathcal{F}}^{\prime}, \overline{f_{*} \mathcal{F}}\right)$, then

$$
T\left(\bar{\xi}_{1}\right)=T(\bar{\xi})+\bar{f}_{\mathrm{b}}\left[\widetilde{\operatorname{ch}}\left(\overline{\mathcal{F}}^{\prime}, \overline{\mathcal{F}}\right)\right] .
$$


(ii) If $\bar{f}^{\prime}$ is another choice of hermitian structure on $f$ and $\bar{\xi}_{2}=\left(\bar{f}^{\prime}, \overline{\mathcal{F}}, \overline{f_{*} \mathcal{F}}\right)$, then

$$
T\left(\bar{\xi}_{2}\right)=T(\bar{\xi})+\bar{f}_{b}^{\prime}\left[\operatorname{ch}(\overline{\mathcal{F}}) \bullet \widetilde{T d}_{m}\left(\bar{f}^{\prime}, \bar{f}\right)\right] .
$$

(iii) If ${\overline{f_{*} \mathcal{F}}}^{\prime}$ is a different choice of metric on $f_{*} \mathcal{F}$, and $\bar{\xi}_{3}=\left(\bar{f}, \overline{\mathcal{F}}, \bar{f}_{*} \mathcal{F}^{\prime}\right)$, then

$$
T\left(\bar{\xi}_{3}\right)=T(\bar{\xi})-\widetilde{\operatorname{ch}}\left({\overline{f_{*} \mathcal{F}}}^{\prime}, \overline{f_{*} \mathcal{F}}\right) .
$$

Proposition 7.6. Let $T$ be a theory of generalized analytic torsion classes. Let $\bar{f}: X \rightarrow Y$ be a morphism in $\overline{\mathbf{S m}}_{* / \mathbb{C}}$. Consider the distinguished triangles in $\overline{\mathbf{D}}^{\mathrm{b}}(X)$ and $\overline{\mathbf{D}}^{\mathrm{b}}(Y)$ respectively:

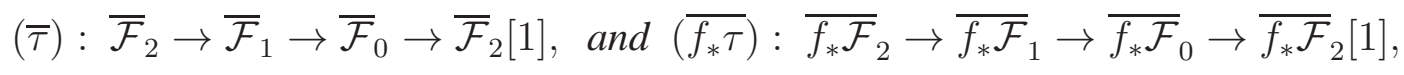

and the relative metrized complexes $\bar{\xi}_{i}=\left(\bar{f}, \overline{\mathcal{F}}_{i}, \bar{f}_{*} \mathcal{F}_{i}\right), i=0,1,2$. Then we have:

$$
\sum_{j=0,1,2}(-1)^{j} T\left(\bar{\xi}_{j}\right)=\widetilde{c h}\left(\overline{\pi_{*} \tau}\right)-\bar{f}_{b}(\widetilde{c h}(\bar{\tau}))
$$

The main result of this section is the following classification theorem.

Theorem 7.7. Let $S$ be a real additive genus. Then there exists a unique theory of generalized analytic torsion classes that agrees with $T_{S}$ when restricted to the class of closed immersions. Moreover, if $T$ is a theory of generalized analytic torsion classes, then there exists a real additive genus $S$ such that $T=T_{S}$.

We will denote the theory associated to the additive genus $S$, whose existence is guaranteed by the preceding theorem, by $T_{S}$. In particular, there is a unique theory of generalized analytic torsion classes that agrees with $T^{h}$ when restricted to the class of closed immersions. This theory will be called homogeneous.

Proof. We first prove the uniqueness. Let $T$ be a theory of analytic torsion classes that agrees with $T_{S}$ for the class of closed immersions. Since the restriction of $T$ to projective spaces, by the transitivity axiom, is compatible with $T_{S}$, by Theorem 6.4, it also agrees with $T_{S}$. Finally, the transitivity axiom implies that $T$ is determined by its values for closed immersions and projective spaces.

We now prove the existence. For the moment, let $T_{S}$ be the theory of analytic torsion classes for closed immersions and projective spaces determined by $S$. Let $\bar{f}: X \rightarrow Y$ be a morphism in $\overline{\mathbf{S m}}_{* / \mathbb{C}}$, and let $\bar{\xi}=\left(\bar{f}, \overline{\mathcal{F}}, \overline{f_{*} \mathcal{F}}\right)$ be a relative metrized complex. Since $f$ is assumed to be projective, there is a factorization $f=\pi \circ \iota$, where $\iota: X \rightarrow \mathbb{P}_{Y}^{n}$ is a closed immersion and $\pi: \mathbb{P}_{Y}^{n} \rightarrow Y$ is the projection. Choose auxiliary hermitian structures on $\iota$, $\pi$ and $\iota_{*} \mathcal{F}$. Then we define

$$
T_{S}(\bar{\xi})=T_{S}(\bar{\pi})+\bar{\pi}_{b}\left(T_{S}(\bar{l})\right)+\bar{f}_{b}\left[\operatorname{ch}(\overline{\mathcal{F}}) \bullet \widetilde{\mathrm{Td}}_{m}(\bar{f}, \bar{\pi} \circ \bar{\iota})\right]
$$

To simplify the notations, in the sequel we will also refer to it simply by $T(\bar{\xi})$. The anomaly formulas easily imply that this definition does not depend on the choice of hermitian structures on $\iota, \pi$ and $\iota_{*} \mathcal{F}$. We next show that this definition is independent of the 
factorization of $f$. Let $f=\pi_{1} \circ \iota_{1}=\pi_{2} \circ \iota_{2}$ be two different factorizations, being $\mathbb{P}^{n_{i}}$, the target of $\iota_{i}, i=1,2$. Since equation (7.8) is independent of the choice of auxiliary hermitian structures, by [17, Lem. 5.12], we may assume that $\bar{f}=\bar{\pi}_{1} \circ \bar{\iota}_{1}=\bar{\pi}_{2} \circ \bar{\iota}_{2}$.

We consider the commutative diagram with cartesian square

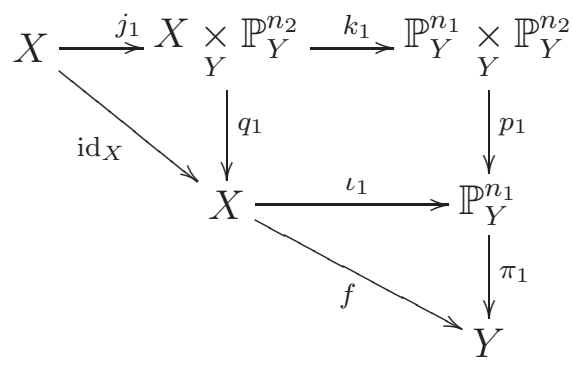

where $j_{1}(x)=\left(x, \iota_{2}(x)\right), p_{1}$ is the first projection and $q_{1}$ and $k_{1}$ are defined by the cartesian square. The hermitian structure of $\bar{\pi}_{2}$ induces a hermitian structure on $p_{1}$ that, in turn, induces a hermitian structure on $q_{1}$. The hermitian structure of $\iota_{1}$ induces a hermitian structure on $k_{1}$ and the hermitian structure of $\iota_{2}$ induces one on $j_{1}$. We will denote the corresponding morphisms of $\overline{\mathbf{S m}}_{* / \mathbb{C}}$ by $\bar{p}_{1}, \bar{q}_{1}, \bar{k}_{1}$ and $\bar{j}_{1}$. We consider also the analogous diagram obtained swapping 1 and 2. Finally, we write $\bar{p}=\bar{\pi}_{1} \circ \bar{p}_{1}=\bar{\pi}_{2} \circ \bar{p}_{2}$ and $\bar{j}=\bar{k}_{1} \circ \bar{j}_{1}=\bar{k}_{2} \circ \bar{j}_{2}$. Then we have

$$
\begin{aligned}
T\left(\bar{\pi}_{1}\right)+\left(\bar{\pi}_{1}\right)_{b}\left(T\left(\bar{\iota}_{1}\right)\right) & =T\left(\bar{\pi}_{1}\right)+\left(\bar{\pi}_{1}\right)_{b}\left(T\left(\bar{\iota}_{1}\right)\right)+\bar{f}_{b}\left(T\left(\bar{q}_{1}\right)+\left(\bar{q}_{1}\right)_{b}\left(T\left(\bar{j}_{1}\right)\right)\right) \\
& =T\left(\bar{\pi}_{1}\right)+\left(\bar{\pi}_{1}\right)_{b}\left(T\left(\bar{\iota}_{1}\right)+\left(\bar{\iota}_{1}\right)_{b}\left(T\left(\bar{q}_{1}\right)\right)\right)+\bar{p}_{b}\left(\bar{k}_{1}\right)_{b}\left(T\left(\bar{j}_{1}\right)\right) \\
& =T\left(\bar{\pi}_{1}\right)+\left(\bar{\pi}_{1}\right)_{b}\left(T\left(\bar{p}_{1}\right)+\left(\bar{p}_{1}\right)_{b}\left(T\left(\bar{k}_{1}\right)\right)\right)+\bar{p}_{b}\left(\bar{k}_{1}\right)_{b}\left(T\left(\bar{j}_{1}\right)\right) \\
& =T(\bar{p})+\bar{p}_{b}(T(\bar{j})) .
\end{aligned}
$$

Analogously, we obtain

$$
T\left(\bar{\pi}_{2}\right)+\left(\bar{\pi}_{2}\right)_{b}\left(T\left(\bar{\iota}_{2}\right)\right)=T(\bar{p})+\bar{p}_{b}(T(\bar{j})) .
$$

Hence $T_{S}$ is well defined for all relative metrized complexes.

It remains to prove that it satisfies the properties of a theory of analytic torsion classes. The properties (i) to (iv) are clear. We thus focus on property (v)] Let $\bar{f}: X \rightarrow Y$ and $\bar{g}: Y \rightarrow Z$ be morphisms in $\overline{\mathbf{S m}}_{* / \mathbb{C}}$. We choose factorizations of $\bar{g} \circ \bar{f}$ and $\bar{g}$ :
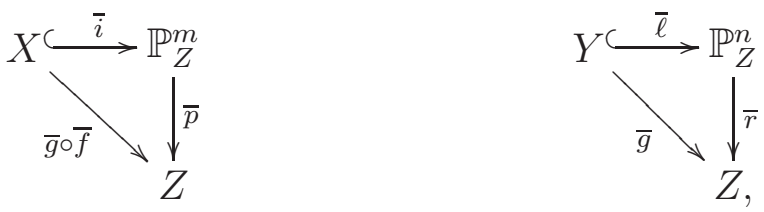

where the hermitian structures on $\bar{p}$ and $\bar{r}$ come from fixed hermitan structures on the tangent bundles $T_{\mathbb{P}_{\mathbb{C}}^{m}}$ and $T_{\mathbb{P}_{\mathbb{C}}^{n}}$, and the hermitian structures $\bar{i}$ and $\bar{\ell}$ are obtained by using [17, Lem. 5.12]. We define $\varphi: X \rightarrow \mathbb{P}_{\mathbb{C}}^{m}$ to be the morphism obtained from $i$ by composing with the projection to $\mathbb{P}_{\mathbb{C}}^{m}$. Then we see that the morphism $j:=(\varphi, f): X \rightarrow \mathbb{P}_{Y}^{m}$ is a closed immersion. Indeed, it is enough to realize that the composition

$$
X \stackrel{(\varphi, f)}{\longrightarrow} \mathbb{P}_{Y}^{m} \stackrel{(\mathrm{id}, g)}{\longrightarrow} \mathbb{P}_{Z}^{m}
$$


agrees with the closed immersion $i$ and that $G:=(\mathrm{id}, g)$ is separated (since proper). We can thus decompose $\bar{f}$ as

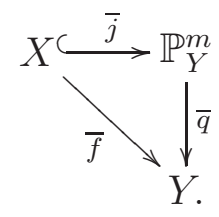

Again, in this factorization the hermitian structure $\bar{q}$ comes from the previously fixed hermitian structure on $T_{\mathbb{P}_{\mathbb{C}}^{m}}$ and the hermitian structure $\bar{j}$ is obtained by using [17, Lem. 5.12]. Because $\bar{g} \circ \bar{f}=\bar{p} \circ \bar{i}$ and by the very construction of $T$ for arbitrary projective morphisms (7.8), we have

$$
T(\bar{g} \circ \bar{f})=T(\bar{p})+\bar{p}_{b}(T(\bar{i})) .
$$

We proceed to work on $T(\bar{i})$. For this we write the commutative diagram

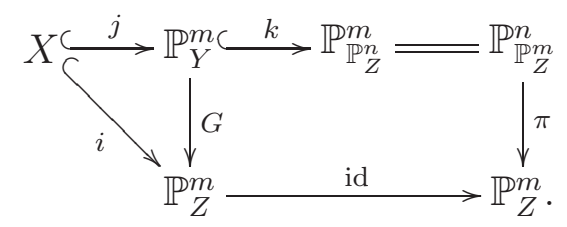

We recall that $G=(\mathrm{id}, g)$ and $k=(\mathrm{id}, \ell)$. Below, $G, k$ and $\pi$ will be endowed with the obvious hermitian structures. With these choices, we observe that $\bar{i}=\bar{G} \circ \bar{j}$ and $\bar{G}=\bar{\pi} \circ \bar{k}$. Taking also into account the construction of $T$ and the fact that $T=T_{S}$ is transitive for compositions of closed immersions, we find

$$
T(\bar{i})=T(\bar{\pi} \circ \bar{k} \circ \bar{j})=T(\bar{\pi})+\bar{\pi}_{b}(T(\bar{k}))+\bar{G}_{b}(T(\bar{j}))=T(\bar{G})+\bar{G}_{b}(T(\bar{j})) .
$$

Therefore, from equations (7.9), (7.10) and the identity $\bar{p}_{b} \bar{G}_{b}=\bar{g}_{b} \bar{q}_{b}$ we derive

$$
T(\bar{g} \circ \bar{f})=T(\bar{p})+\bar{p}_{b}(T(\bar{G}))+\bar{g}_{b} \bar{q}_{b}(T(\bar{j})) .
$$

We claim that

$$
T(\bar{p})+\bar{p}_{b}(T(\bar{G}))=T(\bar{g})+\bar{g}_{b}(T(\bar{q})) .
$$

Assuming this for a while, we combine (7.11) and (7.12) into

$$
T(\bar{g} \circ \bar{f})=T(\bar{g})+\bar{g}_{b}\left(T(\bar{q})+\bar{q}_{b}(T(\bar{j}))\right)=T(\bar{g})+\bar{g}_{b}(T(\bar{f})) .
$$

Hence we are lead to prove (7.12). For this we construct the commutative diagram with cartesian squares

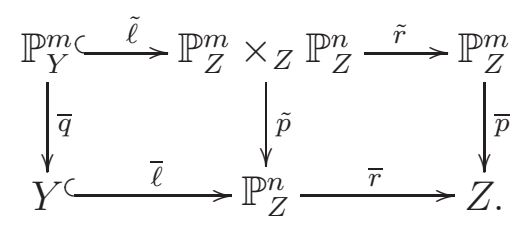

Observe that $\bar{G}=\tilde{r} \circ \tilde{\ell}$. Recall now Proposition 5.41 and Proposition 6.15. We then have the chain of equalities

$$
\begin{aligned}
& T(\bar{p})+\bar{p}_{b}(T(\bar{G}))=T(\bar{p})+\bar{p}_{b}(T\left.(\tilde{r})+\tilde{r}_{b}(T(\tilde{\ell}))\right)=T(\bar{r})+\bar{r}_{b}\left(T(\tilde{p})+\tilde{p}_{b}(T(\tilde{\ell}))\right. \\
&=T(\bar{r})+\bar{r}_{b}\left(T(\bar{\ell})+\bar{\ell}_{b}(T(\bar{q}))\right)=T(\bar{g})+\bar{g}_{b}(T(\bar{q})) .
\end{aligned}
$$


This proves the claim.

The last assertion of the statement of the theorem follows from the uniqueness.

Theorem 7.14. (i) Let $T$ be a theory of generalized analytic torsion classes. Then there is a unique real additive genus $S$ such that, for any relative metrized complex $\bar{\xi}:=\left(\bar{f}, \overline{\mathcal{F}}, \overline{f_{*} \mathcal{F}}\right)$, we have

$$
T(\bar{\xi})-T^{h}(\bar{\xi})=-f_{*}\left[\operatorname{ch}(\mathcal{F}) \bullet \operatorname{Td}\left(T_{f}\right) \bullet S\left(T_{f}\right) \bullet \mathbf{1}_{1}\right] .
$$

(ii) Conversely, any real additive genus $S$ defines, by means of equation (7.15), a unique theory of generalized analytic torsion classes $T_{S}$.

Proof. We prove the first item, the second being immediate. Let $S$ be the real additive genus corresponding to $T$, provided by Theorem 7.7 . Then (7.15) holds for embedded metrized complexes. Because $T$ and $T^{h}$ are both transitive, it suffices to show that (7.15) holds whenever $f: \mathbb{P}_{X}^{n} \rightarrow X$ is a trivial projective bundle. Observe $T$ and $T^{h}$ satisfy the same anomaly formulas. Then, since the sheaves $\mathcal{G}(k), k=-n, \ldots, 0$ form a generating class for $\mathbf{D}^{\mathrm{b}}\left(\mathbb{P}_{X}^{n}\right)$, and by the projection formula for $T$ and $T^{h}$, we easily reduce to the case $\bar{\xi}=\bar{\xi}(k)$. Let $t_{n, k}, t_{n, k}^{h}$ be the characteristic numbers of $T, T^{h}$ respectively. We have to establish the equality

$$
t_{n,-i}-t_{n,-i}^{h}=-\pi_{*}\left(\operatorname{ch}(\overline{\mathcal{O}}(-i)) \operatorname{Td}(\bar{\pi}) S\left(T_{\bar{\pi}}\right)\right), \quad i=-n, \ldots, 0 .
$$

This is an equation of real numbers. By functoriality, this equation is equivalent to the analogous equation in $\oplus_{p} H_{\mathcal{D}}^{2 p-1}\left(\mathbb{P}_{\mathbb{C}}^{n}, \mathbb{R}(p)\right)$, for the second projection $p_{2}: \mathbb{P}_{\mathbb{C}}^{n} \times \mathbb{P}_{\mathbb{C}}^{n} \rightarrow \mathbb{P}_{\mathbb{C}}^{n}$ instead of $\pi$. Because the classes $\operatorname{ch}\left(\Lambda^{i} Q^{\vee}\right)$ constitute a basis for $\oplus_{p} H_{\mathcal{D}}^{2 p-1}\left(\mathbb{P}_{\mathbb{C}}^{n}, \mathbb{R}(p)\right)$, (7.16) is equivalent to the equation in cohomology

$$
\begin{aligned}
\sum_{i}(-1)^{i}\left(t_{n,-i}-\right. & \left.t_{n,-i}^{h}\right) \operatorname{ch}\left(\Lambda^{i} \bar{Q}^{\vee}\right)= \\
& -p_{2 *}\left(\sum_{i}(-1)^{i} \operatorname{ch}\left(p_{1}^{*} \overline{\mathcal{O}}(-i) \otimes \Lambda^{i} p_{2}^{*} \bar{Q}^{\vee}\right) \operatorname{Td}\left(\bar{p}_{2}\right) S\left(T_{\bar{p}_{2}}\right) \bullet \mathbf{1}_{1}\right) .
\end{aligned}
$$

Recalling the exact sequence (6.1), minus the right hand side of (7.17) becomes

$$
\begin{aligned}
& p_{2 *}\left(\operatorname{ch}\left(\overline{\Delta_{*} \mathcal{O}_{\mathbb{P}^{n}}}\right) \operatorname{Td}\left(\bar{p}_{2}\right)\right.\left.S\left(T_{\bar{p}_{2}}\right) \bullet \mathbf{1}_{1}\right)= \\
& p_{2 *}\left(\Delta_{*}\left(\operatorname{ch}\left(\overline{\mathcal{O}}_{\mathbb{P}^{n}}\right) \operatorname{Td}(\bar{\Delta})\right) \operatorname{Td}\left(\bar{p}_{2}\right) S\left(T_{\bar{p}_{2}}\right) \bullet \mathbf{1}_{1}\right)=S\left(T_{\mathbb{P}^{n}}\right) \bullet \mathbf{1}_{1} .
\end{aligned}
$$

On the other hand, using the compatibility condition (Definition 6.2), the left hand side of (7.17) can be equivalently written as

$$
\begin{aligned}
T\left(\bar{p}_{2}, \overline{\Delta_{*} \mathcal{O}_{\mathbb{P}^{n}}}, \overline{\mathcal{O}_{\mathbb{P}^{n}}}\right)-T^{h}\left(\bar{p}_{2}, \overline{\Delta_{*} \mathcal{O}_{\mathbb{P}^{n}}}, \overline{\mathcal{O}_{\mathbb{P}^{n}}}\right)= \\
\quad-p_{2 b}\left(T\left(\Delta, \overline{\mathcal{O}_{\mathbb{P}^{n}}}, \overline{\Delta_{*} \mathcal{O}_{\mathbb{P}^{n}}}\right)-T^{h}\left(\Delta, \overline{\mathcal{O}}_{\mathbb{P}^{n}}, \overline{\Delta_{*} \mathcal{O}_{\mathbb{P}^{n}}}\right)\right) .
\end{aligned}
$$

The genus $S$ is additive, so in Deligne cohomology we have the relation

$$
S\left(T_{\bar{\Delta}}\right)=S\left(T_{\mathbb{P}^{n}}\right)-\Delta^{*} S\left(T_{\mathbb{P}^{n} \times \mathbb{P}^{n}}\right)=S\left(T_{\mathbb{P}^{n}}\right)-\Delta^{*} p_{1}^{*} S\left(T_{\mathbb{P}^{n}}\right)-\Delta^{*} p_{2}^{*} S\left(T_{\mathbb{P}^{n}}\right)=-S\left(T_{\mathbb{P}^{n}}\right) .
$$

Hence, since the statement is known for closed immersions, the right hand side of (7.18) becomes

$$
p_{2 *}\left(\Delta_{*}\left(\operatorname{ch}\left(\overline{\mathcal{O}_{\mathbb{P}^{n}}}\right) \operatorname{Td}\left(T_{\bar{\Delta}}\right) S\left(T_{\bar{\Delta}}\right) \bullet \mathbf{1}_{1}\right) \operatorname{Td}\left(\bar{p}_{2}\right)\right)=-S\left(T_{\mathbb{P}^{n}}\right) \bullet \mathbf{1}_{1} .
$$

This concludes the proof. 


\section{Higher analytic torsion forms of Bismut and Köhler}

We now explain the relationship between the theory of analytic torsion forms of BismutKöhler [13] and the theory of generalized analytic torsion classes developed so far.

Let $\pi: X \rightarrow Y$ be a smooth projective morphism (a projective submersion) of smooth complex varieties. Let $\omega$ be a closed $(1,1)$-form on $X$ that induces a Kähler metric on the fibers of $\pi$. Then $(\pi, \omega)$ is called a Kähler fibration. The form $\omega$ defines a hermitian structure on $T_{\pi}$, and we will abusively write $\bar{\pi}=(\pi, \omega)$ for the corresponding morphism in $\overline{\mathbf{S m}}_{* / \mathbb{C}}$.

Let $\bar{F}$ be a hermitian vector bundle on $X$ such that for every $i \geq 0, R^{i} \pi_{*} F$ is locally free. We consider on $R^{i} \pi_{*} F$ the $L^{2}$ metric obtained using Hodge theory on the fibers of $\pi$. Using [17, Def. 3.47] we obtain a hermitian structure on $\pi_{*} F$, denoted by $\bar{\pi}_{*} F_{L^{2}}$. Then $\bar{\xi}=\left(\bar{\pi}, \bar{F}, \bar{\pi}_{*} F_{L^{2}}\right)$ is a relative metrized complex. The relative metrized complexes that arise in this way will be said to be Kähler.

In the paper [13], Bismut and Köhler associate to every Kähler relative metrized complex $\bar{\xi}$ a differential form, that we temporarily denote by $\tau(\bar{\xi})$. Since in [13] the authors use real valued characteristic classes, while we use characteristic classes in the Deligne complex, we have to change the normalization of this form. To this end, if $\tau(\bar{\xi})^{(p-1, p-1)}$ is the component of degree $(p-1, p-1)$ of $\tau(\bar{\xi})$, then we put

$$
T^{B K}(\bar{\xi})^{(2 p-1, p)}=\frac{1}{2}(2 \pi i)^{p-1}\left[\tau(\bar{\xi})^{(p-1, p-1)}\right] \in \widetilde{\mathcal{D}}_{D}^{2 p-1}(Y, \emptyset, p) .
$$

We recall that $[\cdot]$ converts differential forms into currents according with the conventions in [19, §1] (compare with equation (3.20)). We define

$$
T^{B K}(\bar{\xi})=\sum_{p \geq 1} T^{B K}(\bar{\xi})^{(2 p-1, p)} .
$$

The first main result of [13] is that this class satisfies the differential equation

$$
d_{\mathcal{D}} T^{B K}(\bar{\xi})=\operatorname{ch}\left(\bar{\pi}_{*} L_{L^{2}}\right)-\bar{\pi}_{b}[\operatorname{ch}(\bar{F})] .
$$

Thus, $T^{B K}(\bar{\xi})$ is an example of analytic torsion class.

Let now $\omega^{\prime}$ be another closed $(1,1)$-form on $X$ that induces a Kähler metric on the fibers of $\pi$. We denote $\bar{\pi}^{\prime}=\left(\pi, \omega^{\prime}\right)$. Let $\bar{F}^{\prime}$ be the vector bundle $F$ with another choice of metric and define $\bar{\pi}_{*} F_{L^{2}}^{\prime}$ to be the object $\pi_{*} F$ with the $L^{2}$ metric induced by $\omega^{\prime}$ and $\bar{F}^{\prime}$. We write $\bar{\xi}^{\prime}$ for the Kähler relative metrized complex $\left(\bar{\pi}^{\prime}, \bar{F}^{\prime}, \bar{\pi}_{*}^{\prime}{ }_{L^{2}}^{\prime}\right)$.

The second main result of [13] is the following anomaly formula.

Theorem 8.1 ([13] Theorem 3.10). The following formula holds:

$$
T^{B K}\left(\bar{\xi}^{\prime}\right)-T^{B K}(\bar{\xi})=\widetilde{\operatorname{ch}}\left(\bar{\pi}_{*} L_{L^{2}},{\overline{\pi_{*} F^{2}}}_{L^{2}}\right)+\bar{\pi}_{b}^{\prime}\left[\operatorname{ch}(\bar{F}) \bullet \widetilde{\mathrm{Td}}_{m}\left(\bar{\pi}^{\prime}, \bar{\pi}\right)-\widetilde{\operatorname{ch}}\left(\bar{F}, \bar{F}^{\prime}\right)\right] .
$$

In the book [3], Bismut studies the compatibility of higher analytic torsion forms with complex immersions. Before stating his result we have to recall the definition of the $R$ genus of Gillet and Soulé [29]. It is the additive genus attached to the power series

$$
R(x)=\sum_{\substack{m \text { odd } \\ m \geq 1}}\left(2 \zeta^{\prime}(-m)+\left(1+\frac{1}{2}+\cdots+\frac{1}{m}\right) \zeta(-m)\right) \frac{x^{m}}{m !} .
$$


Let $T_{-R / 2}$ be the theory of analytic torsion classes for closed immersions associated to $\frac{-1}{2} R$.

Remark 8.3. The fact that we obtain the additive genus $-R / 2$ instead of $R$ is due to two facts. The signs comes from the minus sign in equation (3.12), while the factor $1 / 2$ comes from the difference of the normalization of Green forms used in this paper and the one used in [27]. Note however that the arithmetic intersection numbers computed using both normalizations agree, because the definition of arithmetic degree in [27, §3.4.3] has a factor $1 / 2$ while the definition of arithmetic degree in [18, (6.24)] does not.

Consider a commutative diagram of smooth complex varieties

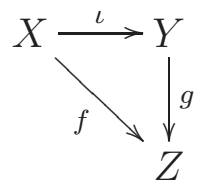

where $f$ and $g$ are projective submersions and $\iota$ is a closed immersion. Let $\bar{F}$ be a hermitian vector bundle on $X$ such that the sheaves $R^{i} f_{*} F$ are locally free and let

$$
0 \rightarrow \bar{E}_{n} \rightarrow \cdots \rightarrow \bar{E}_{0} \rightarrow \iota_{*} F \rightarrow 0
$$

be a resolution of $\iota_{*} F$ by hermitian vector bundles. We assume that for all $i, j, R^{i} g_{*} E_{j}$ is locally free. We will denote by $E$ the complex $E_{n} \rightarrow \cdots \rightarrow E_{0}$. Let $\omega^{X}$ and $\omega^{Y}$ be closed $(1,1)$ forms that define a structure of Kähler fibration on $f$ and $g$ respectively. As before we write $\bar{f}=\left(f, \omega^{X}\right)$ and $\bar{g}=\left(g, \omega^{Y}\right)$. The exact sequence

$$
0 \longrightarrow T_{f} \longrightarrow f^{*} T_{g} \longrightarrow N_{X / Y} \longrightarrow 0
$$

induces a hermitian structure on $N_{X / Y}$. We will denote $\bar{\iota}$ the inclusion $\iota$ with this hermitian structure. Finally we denote by $\bar{f}_{*} F_{E}$ the hermitian structure on $f_{*} F$ induced by the hermitian structures $\overline{g_{*} E_{L^{2}}}, j=0, \ldots, n$.

Then, adapted to our language, the main result of [3] can be stated as follows.

Theorem 8.4 ([3] Theorems 0.1 and 0.2). The following equation holds in the group $\bigoplus_{p} \widetilde{\mathcal{D}}_{D}^{2 p-1}(Z, \emptyset, p)$ :

$$
\begin{aligned}
T^{B K}\left(\bar{f}, \bar{F}, \bar{f}_{*} F_{L_{2}}\right)=\sum_{j=0}^{n}(-1)^{j} T^{B K}\left(\bar{g}, \bar{E}_{j}, \bar{f}_{*} E_{j_{L_{2}}}\right) \\
\\
+\bar{g}_{b}\left(T_{-R / 2}(\bar{l}, \bar{F}, \bar{E})\right)+\widetilde{\operatorname{ch}}\left(\bar{f}_{*} F_{E}, \bar{f}_{*} F_{L^{2}}\right) .
\end{aligned}
$$

We can particularize the previous result to the case when $F=0$. Then $E$ and $g_{*} E$ are acyclic objects. The hermitian structures of $\bar{E}_{j}$ and ${\overline{g_{*}}}_{E_{j L^{2}}}$ induce hermitian structures on them. We denote these hermitian structures as $\bar{E}$ and $\bar{g}_{*} E_{L^{2}}$.

Corollary 8.5. Let $\bar{E}$ be a bounded acyclic complex of hermitian vector bundles on $Y$ such that the direct images $R^{i} g_{*} E_{j}$ are locally free on $Z$. Then

$$
\sum_{j=0}^{n}(-1)^{j} T^{B K}\left(\bar{g}, \bar{E}_{j},{\overline{g_{*} E_{j}}}_{L^{2}}\right)=\widetilde{\operatorname{ch}}\left({\overline{g_{*}}}_{L^{2}}\right)-\bar{g}_{b}(\widetilde{\operatorname{ch}}(\bar{E}))
$$

in $\bigoplus_{p} \widetilde{\mathcal{D}}_{D}^{2 p-1}(Z, \emptyset, p)$. 
We will also need a particular case of functoriality and projection formula for the higher analytic torsion forms of Bismut-Köhler proved by Rössler [43].

The relative metrized complexes $\bar{\xi}_{n}(k)$ of Notation 5.2 are Kähler. Therefore we can apply the construction of Bismut-Köhler to them. We denote

$$
t_{n, k}^{B K}=T^{B K}\left(\bar{\xi}_{n}(k)\right) .
$$

By Corollary 8.5, the numbers $t_{n, k}^{B K}$ satisfy the relation (5.19). Hence they are determined by the main characteristic numbers $t_{n, k}^{B K}$ for $-n \leq k \leq 0$.

Theorem 8.7 ([43] Lemma 7.15). Let $\pi: \mathbb{P}_{X}^{n} \rightarrow X$ be a trivial projective bundle. Let $\bar{G}$ be a hermitian vector bundle on $X$. Then

$$
T^{B K}\left(\bar{\xi}_{n}(k) \otimes \bar{G}\right)=t_{n, k}^{B K} \bullet \operatorname{ch}(\bar{G}) .
$$

Proof. In [43] this result is proved for $k \gg 0$. Using Corollary 8.5 and the Koszul resolution (5.18) one can extend the result to all $k \in \mathbb{Z}$.

We have all the ingredients we need to prove the main result of this section.

Theorem 8.8. Let $T_{-R / 2}$ be the theory of generalized analytic torsion classes associated to the additive genus $\frac{-1}{2} R$. Then, for every Kähler relative metrized complex $\bar{\xi}$, we have

$$
T^{B K}(\bar{\xi})=T_{-R / 2}(\bar{\xi})
$$

In particular $T_{-R / 2}$ extends the construction of Bismut-Köhler to arbitrary projective morphisms of smooth complex varieties and arbitrary smooth metrics.

Proof. Let $\mathfrak{t}^{B K}=\left\{t_{n, k}^{B K} \mid n \geq 0,-n \leq k \leq 0\right\}$ and let $T_{\mathfrak{t}^{B K}}$ be the theory of analytic torsion classes for projective spaces associated to it. Let $\pi: \mathbb{P}_{X}^{n} \rightarrow X$ be a relative projective space and let $\bar{\xi}=\left(\bar{\pi}, \bar{E}, \bar{\pi}_{*} E_{L^{2}}\right)$ be a Kähler relative metrized complex. By choosing $d \gg 0$ we may assume that all the coherent sheaves of the resolution $\gamma_{d}(F)$ of Corollary 4.3 are locally free. Using theorems 8.7 and 8.1, Proposition 5.10 and corollaries 5.15 and 8.5 we obtain that

$$
T^{B K}(\bar{\xi})=T_{\mathfrak{t} B K}(\bar{\xi}) .
$$

By Theorem 8.4 the theories $T_{\mathfrak{t} B K}$ and $T_{-R / 2}$ are compatible in the sense of Definition 6.2. Therefore, $T^{B K}=T_{-R / 2}$ when restricted to projective spaces.

Finally, by factoring a smooth projective morphism as a closed immersion followed by the projection of a relative projective space, Theorem 8.4 implies that $T^{B K}=T_{-R / 2}$ for all smooth projective morphisms.

Remark 8.9. (i) The construction of Bismut-Köhler applies to a wider class of varieties and morphisms: complex analytic manifolds and proper Kähler submersions. However for the comparison we have to restrict to smooth algebraic varieties and smooth projective morphisms.

(ii) The results of Bismut and his coworkers are more precise. Here the class $T^{B K}(\bar{\xi})$ is well defined up to the image of $\mathrm{d}_{\mathcal{D}}$. In contrast, the higher analytic torsion form of Bismut and Köhler is a well defined differential form, local on the base and whose class modulo $\mathrm{d}_{\mathcal{D}}$ agrees with $T^{B K}(\bar{\xi})$. 
As a consequence of Theorem 8.8 , we obtain the following results that, although they should follow from the definition of higher analytic torsion classes, we have not been able to find them explicitly in the literature.

Corollary 8.10. Let $f: X \rightarrow Y$ be a smooth projective morphism of smooth complex varieties, and let $\bar{\xi}=\left(\bar{f}, \bar{E}, \bar{f}_{*} E_{L^{2}}\right)$ be a Kähler relative metrized complex.

(i) Let $g: Y^{\prime} \rightarrow Y$ be a morphism of smooth complex varieties. Then

$$
T^{B K}\left(g^{*} \bar{\xi}\right)=g^{*} T^{B K}(\bar{\xi}) .
$$

(ii) Let $\bar{G}$ be a hermitian vector bundle on $Y$. Then

$$
T^{B K}(\bar{\xi} \otimes \bar{G})=T^{B K}(\bar{\xi}) \bullet \operatorname{ch}(\bar{G}) .
$$

The last consequence we want to discuss generalizes results already proved by Berthomieu-Bismut [1, Thm 3.1] and Ma [35, Thm. 0.1], [36, Thm. 0.1]. However we note that while we stay within the algebraic category and work with projective morphisms, these authors deal with proper Kähler holomorphic submersions of complex manifolds. Let $\bar{g}: X \rightarrow Y$ and $\bar{h}: Y \rightarrow Z$ be morphisms in the category $\overline{\mathbf{S m}}_{* / \mathbb{C}}$, such that the composition $\bar{f}=\bar{h} \circ \bar{g}$ is a smooth morphism. We choose a structure of Kähler fibration on $f$, that we denote $\bar{f}^{\prime}$. Let $\bar{E}$ be a hermitian vector bundle on $X$ and assume that the higher direct images $R^{i} f_{*} E$ are locally free. Then we may consider the analytic torsion $T^{B K}\left(\bar{f}^{\prime}\right)$ attached to the Kähler relative metrized complex $\left(\bar{f}^{\prime}, \bar{E}, \bar{f}_{*} E_{L^{2}}\right)$. Also, we choose an auxiliary hermitian structure on $g_{*} E$. We can consider the torsion classes $T_{R / 2}(\bar{g})$ and $T_{R / 2}(\bar{h})$ of the relative metrized complexes $\left(\bar{g}, \bar{E}, \overline{g_{*} E}\right)$ and $\left(\bar{h}, \overline{g_{*} E}, \bar{f}_{*} E_{L^{2}}\right)$.

We make the following additional assumption in some particular situations:

(*) The morphisms $g$ and $h$ are Kähler fibrations, the higher direct images $R^{i} g_{*} E$ and $R^{j} h_{*} R^{i} g_{*} E$ are locally free and the auxiliary hermitian structure on $g_{*} E$ is the $L^{2}$ hermitian structure.

When the hypothesis (*) is satisfied we denote by $\bar{h}_{*} g_{*} E_{L^{2}}$ the $L^{2}$ hermitian structure attached to the Kähler structure on $\bar{h}$ and the $L^{2}$ metric on $\bar{g}_{*} E_{L^{2}}$. Observe that this last structure may differ from the $L^{2}$ structure on $\bar{f}_{*} E_{L^{2}}$. In this situation we can consider the torsion classes $T^{B K}(\bar{g})$ and $T^{B K}\left(\bar{h}^{\prime}\right)$ attached to $\left(\bar{g}, \bar{E}, \bar{g}_{*} E_{L^{2}}\right)$ and $\left(\bar{h}, \bar{g}_{*} E_{L^{2}}, \bar{h}_{*} g_{*} E_{L^{2}}\right)$. By Proposition 5.10, we have the relation

$$
T^{B K}\left(\bar{h}^{\prime}\right)=T_{R / 2}(\bar{h})-\widetilde{\operatorname{ch}}\left({\overline{h_{*} g_{*} E_{L^{2}}}}_{f_{*} E_{L^{2}}}\right) .
$$

The properties of the generalized analytic torsion classes imply immediately:

Corollary 8.11. Under these assumptions, we have the equality

$$
T^{B K}\left(\bar{f}^{\prime}\right)=T_{-R / 2}(\bar{h})+\bar{h}_{b}\left(T_{-R / 2}(\bar{g})\right)+\bar{f}_{b}^{\prime}\left(\operatorname{ch}(\bar{E}) \bullet \widetilde{T d}_{m}\left(\bar{f}^{\prime}, \bar{f}\right)\right) .
$$

If in addition the hypothesis (*) is satisfied, then we have

$$
T^{B K}\left(\bar{f}^{\prime}\right)=T^{B K}\left(\bar{h}^{\prime}\right)+\bar{h}_{b}\left(T^{B K}(\bar{g})\right)+\bar{f}_{b}^{\prime}\left(\operatorname{ch}(\bar{E}) \bullet \widetilde{\mathrm{Td}}_{m}\left(\bar{f}^{\prime}, \bar{f}\right)\right)+\widetilde{\operatorname{ch}}\left(\bar{h}_{*} g_{*} E_{L^{2}}, \bar{f}_{*} E_{L^{2}}\right) .
$$

Since $T_{-R / 2}$ extends the theory of analytic torsion classes $T^{B K}$, we will denote $T_{-R / 2}$ by $T^{B K}$ for arbitrary relative metrized complexes. 


\section{Grothendieck duality and analytic torsion}

We will study now the compatibility of the analytic torsion with Grothendieck duality.

Definition 9.1. Let $\overline{\mathcal{F}}=(\mathcal{F}, \bar{E} \rightarrow \mathcal{F})$ be an object of $\overline{\mathbf{D}}^{\mathrm{b}}(X)$. Then the rank of $\overline{\mathcal{F}}$ is

$$
\operatorname{rk}(\overline{\mathcal{F}})=\sum_{i}(-1)^{i} \operatorname{dim}\left(E_{i}\right)
$$

This is just the Euler characteristic of the complex. The determinant of $\overline{\mathcal{F}}$ is the complex

$$
\operatorname{det}(\overline{\mathcal{F}})=\bigotimes_{i}\left(\Lambda^{\operatorname{dim} E^{i} \bar{E}^{i}}\right)^{(-1)^{i}}[-\operatorname{rk}(\overline{\mathcal{F}})]
$$

It consists of a single line bundle concentrated in degree $\operatorname{rk}(\overline{\mathcal{F}})$.

Definition 9.2. Let $\bar{f}: X \rightarrow Y$ be a morphism in $\overline{\mathbf{S m}}_{* / \mathbb{C}}$ of relative dimension $e$. The metrized dualizing complex, is the complex given by

$$
\boldsymbol{\omega}_{\bar{f}}=\left(\operatorname{det} T_{\bar{f}}\right)^{\vee} .
$$

This complex is concentrated in degree $-e$. The underlying object of $\mathbf{D}^{\mathrm{b}}(X)$ will be denoted by $\boldsymbol{\omega}_{f}$. If we are interested in the dualizing sheaf as a sheaf and not as an element of $\mathbf{D}^{\mathrm{b}}(X)$ we will denote it by $\omega_{f}$ or $\omega_{X / Y}$. Finally, if $Y=\operatorname{Spec} \mathbb{C}$, we will denote $\boldsymbol{\omega}_{f}$ (respectively $\omega_{f}$ ) by $\omega_{X}$ (respectively $\omega_{X}$ ).

Definition 9.3. Let $\mathcal{D}^{*}(*)$ be the Deligne complex associated to a Dolbeault complex. The sign operator is

$$
\sigma: \mathcal{D}^{*}(*) \longrightarrow \mathcal{D}^{*}(*), \quad \omega \in \mathcal{D}^{n}(p) \mapsto \sigma(\omega)=(-1)^{p} \omega
$$

The sign operator satisfies the following compatibilities.

Proposition 9.4. $\quad$ (i) Let $\left(\mathcal{D}^{*}(*), \mathrm{d}_{\mathcal{D}}\right)$ be a Deligne algebra. Then the sign operator is a morphism of differential algebras. That is

$$
\mathrm{d}_{\mathcal{D}} \circ \sigma=\sigma \circ \mathrm{d}_{\mathcal{D}}, \quad \sigma(\omega \bullet \eta)=\sigma(\omega) \bullet \sigma(\eta) .
$$

(ii) Let $\overline{\mathcal{F}}$ be an object of $\overline{\mathbf{D}}^{\mathrm{b}}(X)$. Then the following equalities are satisfied

$$
\begin{aligned}
\sigma \operatorname{ch}(\overline{\mathcal{F}}) & =\operatorname{ch}\left(\overline{\mathcal{F}}^{\vee}\right), \\
\sigma \operatorname{ch}(\operatorname{det}(\overline{\mathcal{F}})) & =\operatorname{ch}\left(\operatorname{det}(\overline{\mathcal{F}})^{\vee}\right)=\operatorname{ch}(\operatorname{det}(\overline{\mathcal{F}}))^{-1}, \\
\sigma \operatorname{Td}(\overline{\mathcal{F}}) & =(-1)^{\operatorname{rk}(\overline{\mathcal{F}})} \operatorname{Td}(\overline{\mathcal{F}}) \bullet \operatorname{ch}\left(\operatorname{det}(\overline{\mathcal{F}})^{\vee} .\right.
\end{aligned}
$$

Proof. The first statement is clear because if $\omega \in \mathcal{D}^{n}(p)$ and $\eta \in \mathcal{D}^{m}(q)$ then $\mathrm{d}_{\mathcal{D}} \omega \in$ $\mathcal{D}^{n+1}(p)$ and $\omega \bullet \eta \in \mathcal{D}^{n+m}(p+q)$. 
For the second statement, let $\bar{E} \rightarrow-\rightarrow \mathcal{F}$ be the hermitian structure of $\mathcal{F}$. Write

$$
\bar{E}^{+}=\bigoplus_{i \text { even }} \bar{E}^{i}, \quad \bar{E}^{-}=\bigoplus_{i \text { odd }} \bar{E}^{i}
$$

Since this statement is local on $X$, we can chose trivializations of $\bar{E}^{+}$and $\bar{E}^{-}$over an open subset $U$. Let $H^{+}$and $H^{-}$be the matrices of the hermitian metrics on $\bar{E}^{+}$and $\bar{E}^{-}$. The curvature matrices of $\bar{E}^{+}$and $\bar{E}^{-}$, whose entries are elements of $\mathcal{D}^{2}(U, 1)$, are

$$
K^{ \pm}=K^{ \pm}(\overline{\mathcal{F}})=-\bar{\partial}\left(H^{ \pm}\right)^{-1} \partial H^{ \pm} .
$$

The characteristic forms can be computed from the curvature matrix:

$$
\begin{aligned}
\operatorname{ch}(\overline{\mathcal{F}}) & =\operatorname{tr}\left(\exp \left(K^{+}\right)\right)-\operatorname{tr}\left(\exp \left(K^{-}\right)\right), \\
\operatorname{ch}(\operatorname{det}(\overline{\mathcal{F}})) & =(-1)^{\mathrm{rk}(\overline{\mathcal{F}})} \operatorname{det}\left(\exp \left(K^{+}\right)\right) \bullet \operatorname{det}\left(\exp \left(K^{-}\right)\right)^{-1}, \\
\operatorname{Td}(\overline{\mathcal{F}}) & =\operatorname{det}\left(\frac{K^{+}}{1-\exp \left(-K^{+}\right)}\right) \bullet \operatorname{det}\left(\frac{K^{-}}{1-\exp \left(-K^{-}\right)}\right)^{-1} .
\end{aligned}
$$

The sign in the second equation comes from the fact that $\operatorname{det}(\overline{\mathcal{F}})$ is concentrated in degree $\operatorname{rk}(\overline{\mathcal{F}})$. Therefore, since $\sigma\left(K^{ \pm}\right)=-K^{ \pm}=K^{ \pm}\left(\overline{\mathcal{F}}^{\vee}\right)$, we have

$$
\begin{aligned}
\sigma \operatorname{ch}(\overline{\mathcal{F}}) & =\sigma \operatorname{tr}\left(\exp \left(K^{+}\right)\right)-\sigma \operatorname{tr}\left(\exp \left(K^{-}\right)\right) \\
& =\operatorname{tr}\left(\exp \left(K^{+}\left(\overline{\mathcal{F}}^{\vee}\right)\right)\right)-\operatorname{tr}\left(\exp \left(K^{-}\left(\overline{\mathcal{F}}^{\vee}\right)\right)\right)=\operatorname{ch}\left(\overline{\mathcal{F}}^{\vee}\right), \\
\sigma \operatorname{ch}(\operatorname{det}(\overline{\mathcal{F}})) & =\operatorname{det}\left(\exp \left(-K^{+}\right)\right) \bullet \operatorname{det}\left(\exp \left(-K^{-}\right)\right)^{-1}=\operatorname{ch}(\operatorname{det}(\overline{\mathcal{F}}))^{-1}, \\
\sigma \operatorname{Td}(\overline{\mathcal{F}}) & =\operatorname{det}\left(\frac{-K^{+}}{1-\exp \left(K^{+}\right)}\right) \bullet \operatorname{det}\left(\frac{-K^{-}}{1-\exp \left(K^{-}\right)}\right)^{-1} \\
& =\operatorname{det}\left(\frac{K^{+}}{1-\exp \left(-K^{+}\right)}\right) \bullet \operatorname{det}\left(\exp \left(-K^{+}\right)\right) \\
& \bullet \operatorname{det}\left(\frac{K^{-}}{1-\exp \left(-K^{-}\right)}\right)^{-1} \bullet \operatorname{det}\left(\exp \left(-K^{-}\right)\right)^{-1} \\
& =\operatorname{Td}(\overline{\mathcal{F}}) \bullet \operatorname{ch}(\operatorname{det}(\overline{\mathcal{F}}))^{-1} .
\end{aligned}
$$

Corollary 9.8. Let $[\bar{E}] \in \mathbf{K A}(X)$. Then $\widetilde{\operatorname{ch}}\left(\bar{E}^{\vee}\right)=\sigma \widetilde{c h}(\bar{E})$.

Proof. Due to Proposition 9.4 , the assignment sending $[\bar{E}]$ to $\sigma \widetilde{c h}(\bar{E})$ satisfies the characterizing properties of $\widetilde{c h}$.

In the particular case of a projective morphism between smooth complex varieties or, more generally, smooth varieties over a field, Grothendieck duality takes a very simple form (see for instance [32, §3.4] and the references therein). If $\mathcal{F}$ is an object of $\mathbf{D}^{\mathrm{b}}(X)$ and $f: X \rightarrow Y$ is a projective morphism of smooth complex varieties, then there is a natural functorial isomorphism

$$
f_{*}\left(\mathcal{F}^{\vee} \otimes \boldsymbol{\omega}_{f}\right) \cong\left(f_{*} \mathcal{F}\right)^{\vee} .
$$

The compatibility between analytic torsion and Grothendieck duality is given by the following result. 
Theorem Definition 9.10. Let $T$ be a theory of generalized analytic torsion classes. Then the assignment that, to a relative metrized complex $\bar{\xi}=\left(\bar{f}, \overline{\mathcal{F}}, \overline{f_{*}} \mathcal{F}\right)$, associates the class

$$
T^{\vee}(\bar{\xi})=\sigma T\left(\bar{f}, \overline{\mathcal{F}}^{\vee} \otimes \boldsymbol{\omega}_{\bar{f}},{\overline{f_{*} \mathcal{F}^{\vee}}}^{\vee}\right.
$$

is a theory of generalized analytic torsion classes that we call the theory dual to $T$.

Proof. We have to show that, if $T$ satisfies the conditions of Definition 7.1 , then the same is true for $T^{\vee}$. We start with the differential equation. Let $e$ be the relative dimension of $f$.

$$
\begin{aligned}
\mathrm{d}_{\mathcal{D}} T^{\vee}(\bar{\xi}) & =\mathrm{d}_{\mathcal{D}} \sigma T\left(\bar{f}, \overline{\mathcal{F}}^{\vee} \otimes \boldsymbol{\omega}_{\bar{f}},{\overline{f_{*} \mathcal{F}^{\vee}}}^{\vee}\right. \\
& =\sigma \mathrm{d}_{\mathcal{D}} T\left(\bar{f}, \overline{\mathcal{F}}^{\vee} \otimes \boldsymbol{\omega}_{\bar{f}},{\overline{f_{*} \mathcal{F}^{\vee}}}^{\vee}\right. \\
& =\sigma \operatorname{ch}\left({\overline{f_{*}}}^{\vee}\right)-\sigma f_{*}\left[\operatorname{ch}\left(\overline{\mathcal{F}}^{\vee} \otimes \boldsymbol{\omega}_{\bar{f}}\right) \bullet \operatorname{Td}(\bar{f})\right] \\
& =\operatorname{ch}\left(\overline{f_{*} \mathcal{F}}\right)-(-1)^{e} f_{*}\left[\sigma \operatorname{ch}\left(\overline{\mathcal{F}}^{\vee}\right) \bullet \sigma\left(\operatorname{ch}\left(\operatorname{det}\left(T_{\bar{f}}\right)^{\vee}\right) \bullet \operatorname{Td}\left(T_{\bar{f}}\right)\right)\right] \\
& =\operatorname{ch}\left(\overline{f_{*} \mathcal{F}}\right)-f_{*}[\operatorname{ch}(\overline{\mathcal{F}}) \bullet \operatorname{Td}(\bar{f})]
\end{aligned}
$$

The functoriality and the additivity are clear. We next check the projection formula. Let $\overline{\mathcal{G}}$ be an object of $\overline{\mathbf{D}}^{\mathrm{b}}(Y)$. Then

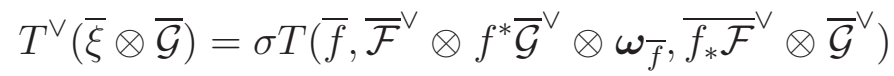

$$
\begin{aligned}
& =\sigma\left(T\left(\bar{f}, \overline{\mathcal{F}}^{\vee} \otimes \boldsymbol{\omega}_{\bar{f}},{\overline{f_{*}}}^{\vee}\right) \bullet \operatorname{ch}\left(\overline{\mathcal{G}}^{\vee}\right)\right)=T^{\vee}(\bar{\xi}) \bullet \operatorname{ch}(\overline{\mathcal{G}}) .
\end{aligned}
$$

Finally we check the transitivity. Let $\bar{g}: Y \rightarrow Z$ be another morphism in $\overline{\mathbf{S m}}_{* / \mathbb{C}}$. By the definition of $\bar{g} \circ \bar{f}$ we have $\boldsymbol{\omega}_{\bar{g} \circ \bar{f}}=f^{*} \boldsymbol{\omega}_{\bar{g}} \otimes \boldsymbol{\omega}_{\bar{f}}$. Therefore,

$$
\begin{aligned}
&\left.f_{*}\left(\mathcal{F}^{\vee} \otimes \boldsymbol{\omega}_{g \circ f}\right)=f_{*}\left(\mathcal{F}^{\vee} \otimes f^{*} \boldsymbol{\omega}_{g} \otimes \boldsymbol{\omega}_{f}\right)\right) \\
&\left.=f_{*}\left(\mathcal{F}^{\vee} \otimes \boldsymbol{\omega}_{f}\right)\right) \otimes \boldsymbol{\omega}_{g}=\left(f_{*} \mathcal{F}\right)^{\vee} \otimes \boldsymbol{\omega}_{g}
\end{aligned}
$$

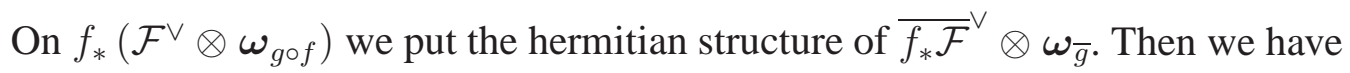

$$
\begin{aligned}
& T^{\vee}(\bar{g} \circ \bar{f})=\sigma T\left(\bar{g} \circ \bar{f}, \overline{\mathcal{F}}^{\vee} \otimes \boldsymbol{\omega}_{\bar{g} \circ \bar{f}}, \overline{(g \circ f)_{*} \mathcal{F}^{\vee}}\right) \\
& =\sigma T\left(\bar{g},{\overline{f_{*} \mathcal{F}}}^{\vee} \otimes \boldsymbol{\omega}_{\bar{g}},{\overline{(g \circ f)_{*} \mathcal{F}}}^{\vee}\right) \\
& +\sigma \bar{g}_{b} T\left(\bar{f}, \overline{\mathcal{F}}^{\vee} \otimes \boldsymbol{\omega}_{\bar{f}} \otimes f^{*} \boldsymbol{\omega}_{\bar{g}},{\overline{f_{*}}}^{\vee} \otimes \boldsymbol{\omega}_{\bar{g}}\right) \\
& =T^{\vee}\left(\bar{g}, \overline{f_{*} \mathcal{F}}, \overline{(g \circ f)_{*} \mathcal{F}}\right) \\
& +\sigma g_{*}\left(T\left(\bar{f}, \overline{\mathcal{F}}^{\vee} \otimes \boldsymbol{\omega}_{\bar{f}},{\overline{f_{*}}}^{\vee} \mathcal{F}^{\vee}\right) \bullet \operatorname{ch}\left(\boldsymbol{\omega}_{\bar{g}}\right) \bullet \operatorname{Td}(\bar{g})\right) \\
& =T^{\vee}(\bar{g})+\bar{g}_{b} T^{\vee}(\bar{f}) \text {. }
\end{aligned}
$$

Therefore, $T^{\vee}$ satisfies also the transitivity property. Hence it is a generalized theory of analytic torsion classes.

Definition 9.11. A theory of generalized analytic torsion classes $T$ is called self-dual when $T^{\vee}=T$. 
We want to characterize the self-dual theories of generalized analytic torsion classes.

Theorem 9.12. The homogeneous theory of generalized analytic torsion classes is selfdual.

Proof. By the uniqueness of the homogeneous theory, it is enough to prove that, if $T$ is homogeneous then $T^{\vee}$ is homogeneous. Let $X$ be a smooth complex variety and let $\bar{N}$ be a hermitian vector bundle of rank $r$ on $X$. Put $P=\mathbb{P}(N \oplus 1)$ and let $s: X \rightarrow P$ be the zero section and $\pi: P \rightarrow X$ the projection. Let $\bar{Q}$ be the tautological quotient bundle with the induced metric and $\bar{K}(s)$ the Koszul resolution associated to the section $s$. Since the normal bundle $N_{X / P}$ can be identified with $N$, on the map $s$ we can consider the hermitian structure given by the hermitian metric on $N$. Then $\operatorname{det} \bar{Q}$ is a complex concentrated in degree $r$. Moreover

$$
s^{*} \operatorname{det} \bar{Q}=\operatorname{det} \bar{N}=\omega_{\bar{s}} \text {. }
$$

The Koszul resolution satisfies the duality property $\bar{K}(s)^{\vee}=\bar{K}(s) \otimes \operatorname{det} \bar{Q}$. The theory $T$ is homogeneous if and only if the class

$$
T\left(\bar{s}, \overline{\mathcal{O}}_{X}, \bar{K}(s)\right) \bullet \operatorname{Td}(\bar{Q})
$$

is homogeneous of bidegree $(2 r-1, r)$ in the Deligne complex. Then

$$
\begin{aligned}
T^{\vee}\left(\bar{s}, \overline{\mathcal{O}}_{X}, \bar{K}(s)\right) \bullet \operatorname{Td}(\bar{Q}) & =\sigma T\left(\bar{s}, \omega_{\bar{s}}, \bar{K}(s)^{\vee}\right) \bullet \operatorname{Td}(\bar{Q}) \\
& =\sigma T\left(\bar{s}, s^{*} \operatorname{det} \bar{Q}, \bar{K}(s) \otimes \operatorname{det} \bar{Q}\right) \bullet \operatorname{Td}(\bar{Q}) \\
& =\sigma\left(T\left(\bar{s}, \overline{\mathcal{O}}_{X}, \bar{K}(s)\right) \bullet \operatorname{ch}(\operatorname{det} \bar{Q})\right) \bullet \operatorname{Td}(\bar{Q}) \\
& =\sigma T\left(\bar{s}, \overline{\mathcal{O}}_{X}, \bar{K}(s)\right) \bullet \operatorname{ch}\left(\operatorname{det} \bar{Q}^{\vee}\right) \bullet \operatorname{Td}(\bar{Q}) \\
& =(-1)^{r} \sigma\left(T\left(\bar{s}, \overline{\mathcal{O}}_{X}, \bar{K}(s)\right) \bullet \operatorname{Td}(\bar{Q})\right)
\end{aligned}
$$

is homogeneous of bidegree $(2 r-1, r)$ in the Deligne complex.

Proposition 9.13. Let

$$
S(x)=\sum_{n=0}^{\infty} a_{n} x^{n} \in \mathbb{R}[[x]]
$$

be a power series in one variable with real coefficients. Denote by $S$ the corresponding real additive genus and by $T_{S}$ the associated theory of analytic torsion classes. Then the dual theory $T_{S}^{\vee}$ has corresponding real additive genus $S^{\sigma}(x):=-S(-x)$.

Proof. Let $\bar{\xi}=\left(\bar{f}, \overline{\mathcal{F}}, \overline{f_{*} \mathcal{F}}\right)$ be a relative metrized complex. If $e$ is the relative dimension of $f$, then we have $\sigma f_{*}=(-1)^{e} f_{*} \sigma$. Then the proposition readily follows from the definition of $T_{S}^{\vee}$, the self-duality of $T^{h}$ and Proposition 9.4 .

We can now characterize the self-dual theories of analytic torsion classes.

Corollary 9.14. The theory of analytic torsion classes $T_{S}$ attached to the real additive genus $S(x)=\sum_{n \geq 0} a_{n} x^{n}$ is self-dual if and only if $a_{n}=0$ for $n$ even.

Proof. By the proposition, $T_{S}^{\vee}=T_{S^{\sigma}}$, hence $T$ is self-dual if, and only if, $S^{\sigma}=S$. The corollary follows. 
In particular we recover the following fact, which is well known if we restrict to Kähler relative metrized complexes.

Corollary 9.15. The theory of analytic torsion classes of Bismut-Köhler $T^{B K}$ is self-dual.

Proof. We just remark that the even coefficients of the $R$-genus vanish (8.2).

We now elaborate on an intimate relation between self-duality phenomena and the analytic torsion of de Rham complexes. Let $f: X \rightarrow Y$ be a smooth projective morphism of smooth algebraic varieties, of relative dimension $e$. Let $\bar{T}_{X / Y}$ denote the vertical tangent bundle, endowed with a hermitian metric. Write $\bar{f}$ for the corresponding morphism in $\overline{\mathbf{S m}}_{* / \mathbb{C}}$. On the locally free sheaves $\Omega_{X / Y}^{p}=\Lambda^{p} \Omega_{X / Y}$ we put the induced hermitian structures. The metrized de Rham complex is

$$
0 \rightarrow \overline{\mathcal{O}}_{X} \stackrel{0}{\rightarrow} \bar{\Omega}_{X / Y} \stackrel{0}{\rightarrow} \bar{\Omega}_{X / Y}^{2} \stackrel{0}{\rightarrow} \ldots \stackrel{0}{\rightarrow} \bar{\Omega}_{X / Y}^{e} \rightarrow 0
$$

with 0 differentials. In fact, we are really considering the de Rham graded sheaf and converting it into a complex in a trivial way. We refer to the corresponding object of $\overline{\mathbf{D}}^{\mathrm{b}}(X)$ by $\bar{\Omega}_{X / Y}^{\bullet}$ ([17, Def. 3.37]). The individual terms $\bar{\Omega}_{X / Y}^{p}$ will be considered as complexes concentrated in degree $p$. We then obviously have:

Lemma 9.16. The objects $\left(\bar{\Omega}_{X / Y}^{\bullet}\right)^{\vee} \otimes \boldsymbol{\omega}_{\bar{f}}$ and $\bar{\Omega}_{X / Y}^{\bullet}[2 e]$ are tightly isomorphic.

For every $p, q$, the cohomology sheaf $R^{q} f_{*} \Omega_{X / Y}^{p}$ is locally free, because the Hodge numbers $h^{p, q}$ of the fibers of $f$ (which are projective, hence Kähler) are known to be locally constant. Every stalk of this sheaf is endowed with the usual $L^{2}$ metric of Hodge theory. This family of $L^{2}$ metrics on $R^{q} f_{*} \Omega_{X / Y}^{p}$ glue into a smooth metric. Because the Hodge star operators $*$ act by isometries, it is easily shown that Serre duality becomes an isometry for the $L^{2}$ structures: the isomorphism

$$
\left(R^{q} f_{*} \Omega_{X / Y}^{p}\right)^{\vee} \stackrel{\sim}{\longrightarrow} R^{e-q} f_{*}\left(\left(\Omega_{X / Y}^{p}\right)^{\vee} \otimes \omega_{f}\right)=R^{e-q} f_{*} \Omega_{X / Y}^{e-p}
$$

preserves the $L^{2}$ hermitian structures. For every $p$, let $\overline{f_{*} \Omega_{X / Y}^{p}}$ denote the object of $\overline{\mathbf{D}}^{\mathrm{b}}(Y)$ with the metric induced by the $L^{2}$ metrics on its cohomology pieces ([17, Def. 3.47]). Here $f_{*}$ stands for the derived direct image. By [17, Prop. 3.48], Grothendieck duality

$$
\left(f_{*} \Omega_{X / Y}^{p}\right)^{\vee} \stackrel{\sim}{\longrightarrow} f_{*} \Omega_{X / Y}^{e-p}[2 e]
$$

is a tight isomorphism. Finally, let $\left[\overline{f_{*} \Omega_{X / Y}^{\bullet}}\right]$ be the object of $\overline{\mathbf{D}}^{\mathrm{b}}(Y)$ provided by [17 Def. 3.39]. The next lemma follows easily from the construction of [17, Def. 3.39].

Lemma 9.17. Grothendieck duality defines a tight isomorphism in $\overline{\mathbf{D}}^{\mathrm{b}}(Y)$

$$
\left[\overline{f_{*} \Omega_{X / Y}^{\bullet}}\right]^{\vee} \cong\left[\overline{f_{*} \Omega_{X / Y}^{\bullet}}\right][2 e] .
$$

Theorem 9.18. Let $T$ be a theory of analytic torsion classes. The following assertions are equivalent: 
(i) the theory $T$ is self-dual;

(ii) for every $f, \bar{T}_{f}, \bar{\Omega}_{X / Y}^{\bullet}$ and $\left[\overline{f_{*} \Omega_{X / Y}^{\bullet}}\right]$ as above and for every odd integer $p \geq 1$, the part of bidegree $(2 p-1, p)$ (in the Deligne complex) of $T\left(\bar{f}, \bar{\Omega}_{X / Y}^{\bullet},\left[\overline{f_{*} \Omega_{X / Y}^{\bullet}}\right]\right.$ ) vanishes.

Proof. Assume first of all that $T$ is self-dual. We apply the definition of $T^{\vee}$, the selfduality assumption and lemmas 9.16 and 9.17 . We find the equality

$$
\begin{aligned}
T\left(\bar{f}, \bar{\Omega}_{X / Y}^{\bullet},\left[\overline{f_{*} \Omega_{X / Y}^{\bullet}}\right]\right) & =\sigma T\left(\bar{f}, \bar{\Omega}_{X / Y}^{\bullet}[2 e],\left[\overline{f_{*} \Omega_{X / Y}^{\bullet}}\right][2 e]\right) \\
& =(-1)^{2 e} \sigma T\left(\bar{f}, \bar{\Omega}_{X / Y}^{\bullet},\left[\overline{f_{*} \Omega_{X / Y}^{\bullet}}\right]\right) \\
& =\sigma T\left(\bar{f}, \bar{\Omega}_{X / Y}^{\bullet},\left[\overline{f_{*} \Omega_{X / Y}^{\bullet}}\right]\right) .
\end{aligned}
$$

The sign operator $\sigma$ changes the sign of the components of bidegree $(2 p-1, p)$ for odd $p$. Hence $T\left(\bar{f}, \bar{\Omega}_{X / Y}^{\bullet},\left[\overline{f_{*} \Omega_{X / Y}^{\bullet}}\right]\right)^{(2 p-1, p)}$ vanishes for $p \geq 1$ odd.

For the converse implication, let $S(x)=\sum_{n>0} a_{n} x^{n}$ be the real additive genus attached to $T$ via Theorem 7.14. By Corollary 9.14, we have to show that the coefficients $a_{n}$ with $n$ even vanish. Let us look at a smooth morphism $f: X \rightarrow Y$ of relative dimension 1 , with an arbitrary metric on $T_{f}$. Then, developing the power series of ch and $\mathrm{Td}$ and taking into account that $\Omega_{X / Y}^{1}=T_{f}^{\vee}=\omega_{X / Y}$, we compute

$$
f_{*}\left[\operatorname{ch}\left(\Omega_{X / Y}^{\bullet} \operatorname{Td}\left(T_{f}\right) S\left(T_{f}\right) \bullet \mathbf{1}_{1}\right]=\sum_{n \geq 0}(-1)^{n+1} a_{n} f_{*}\left[c_{1}\left(\omega_{X / Y}\right)^{n+1} \bullet \mathbf{1}_{1}\right] .\right.
$$

Therefore, for $p \geq 1$ odd, we have

$$
\begin{aligned}
&(-1)^{p} a_{p-1} f_{*} {\left[c_{1}\left(\omega_{X / Y}\right)^{p} \bullet \mathbf{1}_{1}\right]=} \\
&\left(T\left(\bar{f}, \overline{\Omega_{X / Y}^{\bullet}},\left[\overline{f_{*} \Omega_{X / Y}^{\bullet}}\right]\right)-T^{h}\left(\bar{f}, \overline{\Omega_{X / Y}^{\bullet}},\left[\overline{f_{*} \Omega_{X / Y}^{\bullet}}\right]\right)\right)^{(2 p-1, p, p)}=0 .
\end{aligned}
$$

Hence it is enough that for every odd integer $p \geq 1$, we find a relative curve $f: X \rightarrow Y$ such that $f_{*}\left(c_{1}\left(\omega_{X / Y}\right)^{p}\right) \neq 0$ in the cohomology group $H^{2 p}(Y, \mathbb{C})$. Let $d=p-1$ and choose $Y$ to be a smooth projective variety of dimension $d$. Let $L$ be an ample line bundle on $Y$ and take $X=\mathbb{P}\left(L \oplus \mathcal{O}_{Y}\right)$. Consider the tautological exact sequence

$$
0 \longrightarrow \mathcal{O}(-1) \longrightarrow f^{*}\left(L \oplus \mathcal{O}_{Y}\right) \longrightarrow Q \longrightarrow 0 .
$$

We easily derive the relations

$$
\begin{aligned}
& \pi^{*} c_{1}(L)=c_{1}(Q)-c_{1}(\mathcal{O}(1)) \\
& c_{1}(\mathcal{O}(-1)) c_{1}(Q)=0
\end{aligned}
$$

Moreover we have

$$
c_{1}\left(\omega_{X / Y}\right)=-c_{1}(Q)-c_{1}(\mathcal{O}(1)) .
$$

From (9.20)-9.22) and because $d=p-1$ is even, we compute

$$
c_{1}\left(\omega_{X / Y}\right)^{d}=c_{1}(Q)^{d}+c_{1}(\mathcal{O}(1))^{d}=\pi^{*} c_{1}(L)^{d} .
$$


Therefore we find

$$
c_{1}\left(\omega_{X / Y}\right)^{p}=\pi^{*} c_{1}(L)^{d} c_{1}\left(\omega_{X / Y}\right) .
$$

Finally, $f$ is a fibration in curves of genus 0 , hence $f_{*}\left(c_{1}\left(\omega_{X / Y}\right)\right)=-2$. We infer that (9.23) leads to

$$
f_{*}\left(c_{1}\left(\omega_{X / Y}\right)^{p}\right)=-2 c_{1}(L)^{d} .
$$

This class does not vanish, since $Y$ is projective of dimension $d$ and $L$ is ample.

We end with a characterization of the theory of analytic torsion classes of BismutKöhler.

Theorem 9.24. The theory of analytic torsion classes of Bismut-Köhler $T^{B K}$ is the unique theory of generalized analytic torsion classes such that, for every $\bar{f}: X \rightarrow Y$, a Kähler fibration in $\overline{\mathbf{S m}}_{* / \mathbb{C}}$, we have the vanishing

$$
T^{B K}\left(\bar{f}, \bar{\Omega}_{X / Y}^{\bullet},\left[\overline{f_{*} \Omega_{X / Y}^{\bullet}}\right]\right)=0 .
$$

Proof. That the theory $T^{B K}$ vanishes for de Rham complexes of Kähler fibrations is a theorem of Bismut [5]. For the uniqueness, let $T$ be a theory of generalized analytic torsion classes vanishing on de Rham complexes of Kähler fibrations. Denote by $S(x)=$ $\sum_{k \geq 0} a_{k} x^{k}$ its corresponding genus. If $\bar{f}$ is a relative curve with a structure of Kähler fibration, then by Theorem 7.14

$$
T^{h}\left(\bar{f}, \bar{\Omega}_{X / Y}^{\bullet},\left[\overline{f_{*} \Omega_{X / Y}^{\bullet}}\right]\right)=\sum_{k \geq 0}(-1)^{k} a_{k} f_{*}\left[c_{1}\left(\omega_{X / Y}\right)^{k+1} \bullet \mathbf{1}_{1}\right]
$$

It is enough to find, for every $k \geq 0$, a relative curve $f$ such that $f_{*}\left(c_{1}\left(\omega_{X / Y}\right)^{k+1}\right)$ does not vanish. The elementary construction in the proof of Theorem 9.18 works whenever $k$ is even, but one easily sees it fails for $k$ odd. Fortunately, there is an alternative argument. Let $g \geq 2$ and $n \geq 3$ be integers. Consider the fine moduli scheme of smooth curves of genus $g$ with a Jacobi structure of level $n$ [21, Def. 5.4], to be denoted $\mathcal{M}_{g}^{n}$. Let $\pi: \mathcal{C}_{g}^{n} \rightarrow \mathcal{M}_{g}^{n}$ be the universal curve. An example of Kähler fibration structure on $\pi$ is provided by Teichmüller theory (see for instance [48, Sec. 5]). By [22, Thm. 1], the tautological class $\kappa_{g-2}:=\pi_{*}\left(c_{1}\left(\omega_{\pi}\right)^{g-1}\right) \in H^{2(g-2)}\left(\mathcal{M}_{g}^{n}, \mathbb{C}\right)$ does not vanish. Taking $g=k+2$ and $f=\pi$, we conclude the proof of the theorem.

We note that in the previous theorem, the existence is provided by Bismut's theorem. It would be interesting to have a proof of the existence of a theory satisfying the condition of Theorem 9.24 from the axiomatic point of view.

\section{Direct images of hermitian structures}

As another application of a theory of generalized analytic torsion classes, we construct direct images of metrized complexes. It turns out that the natural place to define direct images is not the category $\overline{\mathbf{D}}^{\mathrm{b}}(\cdot)$ but a new category $\widehat{\mathbf{D}}^{\mathrm{b}}(\cdot)$ that is the analogue to the arithmetic $K$-theory of Gillet and Soulé [28]. 
Let $X$ be a smooth complex variety. The fibers of the forgetful functor $\overline{\mathbf{D}}^{\mathrm{b}}(X) \rightarrow$ $\mathbf{D}^{\mathrm{b}}(X)$ have a structure of $\mathbf{K A}(X)$-torsor, for the action of $\mathbf{K A}(X)$ by translation of the hermitian structures ([17, Thm. 3.13]). At the same time, the group $\mathbf{K A}(X)$ acts on the group $\oplus_{p} \widetilde{\mathcal{D}}_{D}^{2 p-1}(X, p)$ by translation, via the Bott-Chern character ch ([17, Prop. 4.6]). Observe that all Bott-Chern classes live in these groups, as for the analytic torsion classes. It is thus natural to build a product category over $\mathbf{K A}(X)$.

Definition 10.1. Let $S \subset T^{*} X_{0}$ be a closed conical subset. We define

$$
\widehat{\mathbf{D}}^{\mathrm{b}}(X, S)=\overline{\mathbf{D}}^{\mathrm{b}}(X) \times_{\mathbf{K A}(X)} \bigoplus_{p} \widetilde{\mathcal{D}}_{D}^{2 p-1}(X, S, p)
$$

to be the category whose objects are equivalence classes $[\overline{\mathcal{F}}, \eta]$ of pairs $(\overline{\mathcal{F}}, \eta)$ belonging to $\mathrm{Ob} \overline{\mathbf{D}}^{\mathrm{b}}(X) \times \oplus_{p} \widetilde{\mathcal{D}}_{D}^{2 p-1}(X, S, p)$, under the equivalence relation

$$
(\overline{\mathcal{F}}, \eta) \sim(\overline{\mathcal{F}}+[\bar{E}], \eta-\widetilde{\operatorname{ch}}(\bar{E}))
$$

for $[\bar{E}] \in \mathbf{K A}(X)$, and with morphisms

$$
\operatorname{Hom}_{\widehat{\mathbf{D}}^{\mathrm{b}}(X)}([\overline{\mathcal{F}}, \eta],[\overline{\mathcal{G}}, \nu])=\operatorname{Hom}_{\mathbf{D}^{\mathrm{b}}(X)}(\mathcal{F}, \mathcal{G}) .
$$

If $S \subset T$ are closed conical subsets of $T^{*} X_{0}$, then $\widehat{\mathbf{D}}^{\mathrm{b}}(X, S)$ is naturally a full subcategory of $\widehat{\mathbf{D}}^{\mathrm{b}}(X, T)$.

In the sequel, we extend the main operations in $\mathbf{D}^{\mathrm{b}}(X)$ to the categories $\widehat{\mathbf{D}}^{\mathrm{b}}(X, S)$. In particular, we use the theory of generalized analytic torsion classes to construct pushforward morphisms attached to morphisms in $\overline{\mathbf{S m}}_{* / \mathbb{C}}$.

The category $\widehat{\mathbf{D}}^{\mathrm{b}}(X, S)$ has a natural additive structure. More generally, if $S, T$ are closed conical subsets of $T^{*} X_{0}$, then there is an obvious addition functor

$$
\widehat{\mathbf{D}}^{\mathrm{b}}(X, S) \times \widehat{\mathbf{D}}^{\mathrm{b}}(X, T) \stackrel{\oplus}{\longrightarrow} \widehat{\mathbf{D}}^{\mathrm{b}}(X, S \cup T) .
$$

The object $[\overline{0}, 0]$ is a neutral element for this operation. If $S+T$ is disjoint with the zero section in $T^{*} X$, then there is a product defined by the functor

$$
\begin{aligned}
& \operatorname{Ob} \widehat{\mathbf{D}}^{\mathrm{b}}(X, S) \times \mathrm{Ob} \widehat{\mathbf{D}}^{\mathrm{b}}(X, T) \stackrel{\otimes}{\longrightarrow} \mathrm{Ob}^{\mathrm{b}}(X,(S+T) \cup S \cup T) \\
&([\overline{\mathcal{F}}, \eta],[\overline{\mathcal{G}}, \nu]) \longmapsto\left[\overline{\mathcal{F}} \otimes \overline{\mathcal{G}}, \operatorname{ch}(\overline{\mathcal{F}}) \bullet \nu+\eta \bullet \operatorname{ch}(\overline{\mathcal{G}})+\mathrm{d}_{D} \eta \bullet \nu\right]
\end{aligned}
$$

and the obvious assignment for morphisms. This product is commutative up to natural isomorphism. It induces on $\widehat{\mathrm{D}}^{\mathrm{b}}(X, \emptyset)$ a structure of commutative and associative ring category. Also, $\left[\overline{\mathcal{O}}_{X}, 0\right]$ is a unity object for the product structure. More generally, the category $\widehat{\mathbf{D}}^{\mathrm{b}}(X, S)$ becomes a left and right $\widehat{\mathbf{D}}^{\mathrm{b}}(X, \emptyset)$ module. Under the same assumptions on $S, T$ we may define an internal Hom. For this, let $[\overline{\mathcal{F}}, \eta] \in \operatorname{Ob}^{\mathrm{b}}(X, S)$ and $[\overline{\mathcal{G}}, \nu] \in \mathrm{Ob} \widehat{\mathbf{D}}^{\mathrm{b}}(X, T)$. Then we put

$$
\underline{\operatorname{Hom}}([\overline{\mathcal{F}}, \eta],[\overline{\mathcal{G}}, \nu])=\left[\underline{\operatorname{Hom}}(\overline{\mathcal{F}}, \overline{\mathcal{G}}),(\sigma \operatorname{ch}(\overline{\mathcal{F}})) \bullet \nu+(\sigma \eta) \bullet \operatorname{ch}(\overline{\mathcal{G}})+\left(\mathrm{d}_{D} \sigma \eta\right) \bullet \nu\right],
$$


where we recall that $\sigma$ is the sign operator (Definition 9.3). Using Corollary 9.8, it is easily seen this is well defined. In particular, we put

$$
[\overline{\mathcal{F}}, \eta]^{\vee}:=\underline{\operatorname{Hom}}\left([\overline{\mathcal{F}}, \eta],\left[\overline{\mathcal{O}}_{X}, 0\right]\right)=\left[\overline{\mathcal{F}}^{\vee}, \sigma \eta\right] .
$$

The shift [1] on $\overline{\mathbf{D}}^{\mathrm{b}}(X)$ induces a well defined shift functor on $\widehat{\mathbf{D}}^{\mathrm{b}}(X, S)$, whose action on objects is

$$
[\overline{\mathcal{F}}, \eta][1]=[\overline{\mathcal{F}}[1],-\eta]
$$

There is a Chern character

$$
\operatorname{ch}: \operatorname{Ob} \widehat{\mathbf{D}}^{\mathrm{b}}(X, S) \longrightarrow \bigoplus_{p} \widetilde{\mathcal{D}}_{D}^{2 p}(X, S, p), \quad[\overline{\mathcal{F}}, \eta] \longmapsto \operatorname{ch}(\overline{\mathcal{F}})+\mathrm{d}_{D} \eta
$$

which is well defined because $\mathrm{d}_{D} \widetilde{\operatorname{ch}}(\bar{E})=\operatorname{ch}(\bar{E})$ for $[\bar{E}] \in \mathbf{K A}(X)$. The Chern character is additive and compatible with the product structure:

$$
\operatorname{ch}([\overline{\mathcal{F}}, \eta] \otimes[\overline{\mathcal{G}}, \nu])=\operatorname{ch}([\overline{\mathcal{F}}, \eta]) \bullet \operatorname{ch}([\overline{\mathcal{G}}, \nu])
$$

Notice the relations

$$
\begin{aligned}
& \operatorname{ch}\left([\overline{\mathcal{F}}, \eta]^{\vee}\right)=\sigma \operatorname{ch}([\overline{\mathcal{F}}, \eta]), \\
& \operatorname{ch}([\overline{\mathcal{F}}, \eta][1])=-\operatorname{ch}([\overline{\mathcal{F}}, \eta]) .
\end{aligned}
$$

We may also define Bott-Chern classes for isomorphisms and distinguished triangles. Let $\widehat{\varphi}:[\overline{\mathcal{F}}, \eta] \rightarrow[\overline{\mathcal{G}}, \nu]$ be an isomorphism in $\widehat{\mathrm{D}}^{\mathrm{b}}(X, S)$, whose underlying morphism in $\mathrm{D}^{\mathrm{b}}(X)$ we denote $\varphi$. While the class $\widetilde{c h}(\varphi: \overline{\mathcal{F}} \rightarrow-\overline{\mathcal{G}})$ depends on the representatives $(\overline{\mathcal{F}}, \eta),(\overline{\mathcal{G}}, \nu)$, the class

$$
\widetilde{\operatorname{ch}}(\widehat{\varphi}):=\widetilde{\operatorname{ch}}(\varphi: \overline{\mathcal{F}} \rightarrow \overline{\mathcal{G}})+\nu-\eta
$$

is well defined.

Lemma 10.3. Let $\widehat{\varphi}:[\overline{\mathcal{F}}, \eta] \rightarrow[\overline{\mathcal{G}}, \nu]$ be an isomorphism in $\widehat{\mathbf{D}}^{\mathrm{b}}(X, S)$, with underlying morphism $\varphi$ in $\mathrm{D}^{\mathrm{b}}(X)$. Then, the following conditions are equivalent:

(i) there exists $[\bar{E}] \in \mathbf{K A}(X)$ such that $\varphi$ induces a tight isomorphism between $\overline{\mathcal{F}}+[\bar{E}]$ and $\overline{\mathcal{G}}$, and $\nu=\eta-\widetilde{\operatorname{ch}}(\bar{E})$;

(ii) $\widetilde{\operatorname{ch}}(\widehat{\varphi})=0$

Proof. This is actually a tautology. Because $\mathbf{K A}(X)$ acts freely and transitively on the possible hermitian structures on $\mathcal{F}$, there exists a unique $[\bar{E}] \in \mathbf{K} \mathbf{A}(X)$ such that $\overline{\mathcal{F}}+[\bar{E}]$ is tightly isomorphic to $\overline{\mathcal{G}}$ via the morphism $\varphi$. Then we have

$$
\widetilde{\operatorname{ch}}(\widehat{\varphi})=\widetilde{\operatorname{ch}}(\bar{E})+\nu-\eta
$$

The lemma follows. 
Definition 10.4. Let $\widehat{\varphi}$ be an isomorphism in $\widehat{\mathbf{D}}^{\mathrm{b}}(X, S)$. We say that $\widehat{\varphi}$ is tight if the equivalent conditions of Lemma 10.3 are satisfied.

In particular, if $\varphi: \overline{\mathcal{F}} \rightarrow \overline{\mathcal{G}}$ is a tight isomorphism in $\overline{\mathbf{D}}^{\mathrm{b}}(X)$, then $\varphi$ induces a tight isomorphism $[\overline{\mathcal{F}}, \eta] \rightarrow[\overline{\mathcal{G}}, \nu]$ if and only if $\eta=\nu$.

The following lemma provides an example involving the notion of tight isomorphism.

Lemma 10.5. Let $[\overline{\mathcal{F}}, \eta] \in \widehat{\mathbf{D}}^{\mathrm{b}}(X, S)$ and $[\overline{\mathcal{G}}, \nu] \in \widehat{\mathbf{D}}^{\mathrm{b}}(X, T)$. Assume that $S+T$ does not cross the zero section. Then there is a functorial tight isomorphism

$$
[\overline{\mathcal{F}}, \eta]^{\vee} \otimes[\overline{\mathcal{G}}, \nu] \cong \underline{\operatorname{Hom}}([\overline{\mathcal{F}}, \eta],[\overline{\mathcal{G}}, \nu]) .
$$

Assume now given a distinguished triangle

$$
\widehat{\tau}: \quad[\overline{\mathcal{F}}, \eta] \rightarrow[\overline{\mathcal{G}}, \nu] \rightarrow[\overline{\mathcal{H}}, \mu] \rightarrow[\overline{\mathcal{F}}, \eta][1] .
$$

Let $\bar{\tau}$ denote the distinguished triangle $\overline{\mathcal{F}} \rightarrow-\rightarrow \overline{\mathcal{G}} \rightarrow \overline{\mathcal{H}} \rightarrow$ in $\overline{\mathbf{D}}^{\mathrm{b}}(X)$. Then we put

$$
\widetilde{\operatorname{ch}}(\widehat{\tau})=\widetilde{\operatorname{ch}}(\bar{\tau})+\eta-\nu+\mu
$$

By [17, Thm. 3.33 (vii)], this class does not depend on the representatives and is thus well defined.

We study now the functoriality of $\widehat{\mathbf{D}}^{\mathrm{b}}(X, S)$ with respect to inverse and direct images. Let $f: X \rightarrow Y$ be a morphism of smooth complex varieties. Let $T \subset T^{*} Y_{0}$ be a closed conical subset disjoint with $N_{f}$. The action of the left inverse image functor on objects is

$$
f^{*}: \mathrm{Ob} \widehat{\mathbf{D}}^{\mathrm{b}}(Y, T) \longrightarrow \mathrm{Ob} \widehat{\mathbf{D}}^{\mathrm{b}}\left(X, f^{*} T\right), \quad[\overline{\mathcal{F}}, \eta] \longmapsto\left[f^{*} \overline{\mathcal{F}}, f^{*} \eta\right] .
$$

That this assignment is well defined amounts to the functoriality of $\widetilde{c h}$.

Let $\bar{f}$ be a morphism in the category $\overline{\mathbf{S m}}_{* / \mathbb{C}}$. The definition of a direct image functor attached to $\bar{f}$ depends upon the choice of a theory of generalized analytic torsion classes. Let $T$ be such a theory. Then we define a functor $\bar{f}_{*}$ whose action on objects is

$$
\begin{aligned}
\bar{f}_{*}: \operatorname{Ob} \widehat{\mathbf{D}}^{\mathrm{b}}(X, S) & \longrightarrow \mathrm{Ob}^{\mathrm{b}}\left(Y, f_{*} S\right) \\
{[\overline{\mathcal{F}}, \eta] } & \longmapsto\left[\overline{f_{*} \mathcal{F}}, \bar{f}_{\mathrm{b}}(\eta)-T\left(\bar{f}, \overline{\mathcal{F}}, \overline{f_{*} \mathcal{F}}\right)\right]
\end{aligned}
$$

where $\overline{f_{*} \mathcal{F}}$ is an arbitrary choice of hermitian structure on $f_{*} \mathcal{F}$. By the anomaly formulas, this definition does not depend on the representative $(\overline{\mathcal{F}}, \eta)$ nor on the choice of hermitian structure on $\overline{f_{*} \mathcal{F}}$.

Theorem 10.7. Let $\bar{f}: X \rightarrow Y$ and $\bar{g}: Y \rightarrow Z$ be morphisms in $\overline{\mathbf{S m}}_{* / \mathbb{C}}$. Let $S \subset T^{*} X_{0}$ and $T \subset T^{*} Y_{0}$ be closed conical subsets.

(i) Let $[\overline{\mathcal{F}}, \eta] \in \mathrm{Ob} \widehat{\mathrm{D}}^{\mathrm{b}}(X, S)$. Then there is a functorial tight isomorphism

$$
(\bar{g} \circ \bar{f})_{*}([\overline{\mathcal{F}}, \eta]) \cong \bar{g}_{*} \bar{f}_{*}([\overline{\mathcal{F}}, \eta]) .
$$


(ii) (Projection formula) Assume that $T \cap N_{f}=\emptyset$ and that $T+f_{*} S$ does not cross the zero section of $T^{*} Y$. Let $[\overline{\mathcal{F}}, \eta] \in \mathrm{Ob} \widehat{\mathbf{D}}^{\mathrm{b}}(X, S)$ and $[\overline{\mathcal{G}}, \nu] \in \mathrm{Ob} \widehat{\mathbf{D}}^{\mathrm{b}}(Y, T)$. Then there is a functorial tight isomorphism

$$
\bar{f}_{*}\left([\overline{\mathcal{F}}, \eta] \otimes f^{*}[\overline{\mathcal{G}}, \nu]\right) \cong \bar{f}_{*}[\overline{\mathcal{F}}, \eta] \otimes[\overline{\mathcal{G}}, \nu]
$$

in $\widehat{\mathbf{D}}^{\mathrm{b}}(Y, W)$, where $W=f_{*}\left(S+f^{*} T\right) \cup f_{*} S \cup f_{*} f^{*} T$.

(iii) (Base change) Consider a cartesian diagram

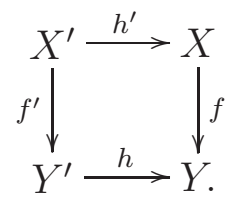

Suppose that $f$ and $h$ are transverse and that $N_{h^{\prime}}$ is disjoint with $S$. Equip $f^{\prime}$ with the hermitian structure induced by the natural isomorphism $h^{*} T_{f} \rightarrow T_{f^{\prime}}$. Let $[\overline{\mathcal{F}}, \eta] \in \mathrm{Ob}_{\mathrm{D}^{\mathrm{b}}}(X, S)$. Then there is a functorial tight isomorphism

$$
h^{*} \bar{f}_{*}[\overline{\mathcal{F}}, \eta] \cong \bar{f}_{*}^{\prime} h^{\prime *}[\overline{\mathcal{F}}, \eta]
$$

in $\widehat{\mathbf{D}}^{\mathrm{b}}\left(Y^{\prime}, f_{*}^{\prime} h^{\prime *} S\right)$.

Proof. The first and the second assertions follow from Proposition 2.14 the transitivity and the projection formula for $T$. For the third item, one uses the functoriality of the analytic torsion classes and Proposition 2.15.

We close this section with an extension of Grothendieck duality to $\widehat{\mathbf{D}}^{\mathrm{b}}$. Let $\bar{f}: X \rightarrow Y$ be a morphism is $\overline{\mathbf{S m}}_{* / \mathbb{C}}$. To enlighten notations, we denote by $\boldsymbol{\omega}_{\bar{f}}$ the object $\left[\boldsymbol{\omega}_{\bar{f}}, 0\right]$ in $\widehat{\mathbf{D}}^{\mathrm{b}}(X, \emptyset)$ (Definition 9.2). Suppose given a closed conical subset $T \subset T^{*} Y_{0}$ such that $T \cap N_{f}=\emptyset$. Then we define the functor $\bar{f}$ ' whose action on objects is

$$
\bar{f}^{!}: \mathrm{Ob} \widehat{\mathbf{D}}^{\mathrm{b}}(Y, T) \longrightarrow \mathrm{Ob} \widehat{\mathbf{D}}^{\mathrm{b}}\left(X, f^{*} T\right), \quad[\overline{\mathcal{F}}, \eta] \longmapsto f^{*}[\overline{\mathcal{F}}, \eta] \otimes \boldsymbol{\omega}_{\bar{f}} .
$$

Observe the equality

$$
[\overline{\mathcal{G}}, \nu] \otimes \boldsymbol{\omega}_{\bar{f}}=\left[\overline{\mathcal{G}} \otimes \boldsymbol{\omega}_{\bar{f}}, \nu \bullet \operatorname{ch}\left(\boldsymbol{\omega}_{\bar{f}}\right)\right] .
$$

Now fix a theory of generalized analytic torsion classes. To the morphism $\bar{f}$ we have attached the direct image functor $\bar{f}_{*}$. We denote by $\bar{f}_{*}^{\vee}$ the direct image functor associated to $\bar{f}$ and the dual theory (Theorem Definition 9.10).

Theorem 10.9 (Grothendieck duality for $\widehat{\mathbf{D}}^{\mathrm{b}}$ ). Let $\bar{f}: X \rightarrow Y$ be a morphism in $\overline{\mathbf{S m}}_{* / \mathbb{C}}$. Let $S \subset T^{*} X_{0}$ and $T \subset T^{*} Y_{0}$ be closed conical subsets such that $T \cap N_{f}=\emptyset$ and $T+f_{*} S$ is disjoint with the zero section. Let $[\overline{\mathcal{F}}, \eta] \in \mathrm{Ob}_{\mathbf{D}^{\mathrm{b}}}(X, S)$ and $[\overline{\mathcal{G}}, \sigma] \in \mathrm{Ob} \widehat{\mathbf{D}}^{\mathrm{b}}(Y, T)$. Then there is a functorial tight isomorphism

$$
\underline{\operatorname{Hom}}\left(\bar{f}_{*}[\overline{\mathcal{F}}, \eta],[\overline{\mathcal{G}}, \nu]\right) \cong \bar{f}_{*}^{\vee} \underline{\operatorname{Hom}}\left([\overline{\mathcal{F}}, \eta], \bar{f}^{!}[\overline{\mathcal{G}}, \nu]\right)
$$

in $\widehat{\mathbf{D}}^{\mathrm{b}}(Y, W)$, where $W=f_{*}\left(S+f^{*} T\right) \cup f_{*} S \cup f_{*} f^{*} T$. 
In particular, we have

$$
\left(\bar{f}_{*}[\overline{\mathcal{F}}, \eta]\right)^{\vee} \cong \bar{f}_{*}^{\vee}\left([\overline{\mathcal{F}}, \eta]^{\vee} \otimes \boldsymbol{\omega}_{\bar{f}}\right) .
$$

Proof. By Lemma 10.5 and Proposition 10.7, we are reduced to establish the functorial tight isomorphism (10.10). The proof follows readily from the definitions, Grothendieck duality and the following two observations. First of all, if $T$ is the theory of analytic torsion classes, then by the very definition of $T^{\vee}$ we find

$$
\sigma T\left(\bar{f}, \overline{\mathcal{F}}, \overline{f_{*} \mathcal{F}}\right)=T^{\vee}\left(\bar{f}, \overline{\mathcal{F}}^{\vee} \otimes \boldsymbol{\omega}_{\bar{f}}, \overline{f_{*}\left(\mathcal{F}^{\vee} \otimes \boldsymbol{\omega}_{f}\right)}\right)
$$

where the metric on $\overline{f_{*}\left(\mathcal{F}^{\vee} \otimes \boldsymbol{\omega}_{f}\right)}$ is chosen so that Grothendieck duality provides a tight isomorphism

$$
{\overline{f_{*}}}^{\vee} \cong \overline{f_{*}\left(\mathcal{F}^{\vee} \otimes \boldsymbol{\omega}_{f}\right)} .
$$

Secondly, for direct images of currents, we compute

$$
\sigma \bar{f}_{b}(\eta)=\sigma f_{*}\left(\eta \bullet \operatorname{Td}\left(T_{\bar{f}}\right)\right)=(-1)^{e} f_{*}\left(\sigma \eta \bullet \sigma \operatorname{Td}\left(T_{\bar{f}}\right)\right)=f_{*}\left(\sigma \eta \bullet \operatorname{ch}\left(\boldsymbol{\omega}_{\bar{f}}\right) \bullet \operatorname{Td}\left(T_{\bar{f}}\right)\right) .
$$

Here $e$ is the relative dimension of $f$, and to derive the last equality we appeal to Proposition 9.4. To conclude, we recall equation 10.8.

Corollary 10.11. Let $T$ be a self-dual theory of generalized analytic torsion classes.

(i) Then there is a functorial isomorphism $\left(\bar{f}_{*}[\overline{\mathcal{F}}, \eta]\right)^{\vee} \cong \bar{f}_{*}\left([\overline{\mathcal{F}}, \eta]^{\vee} \otimes \boldsymbol{\omega}_{\bar{f}}\right)$.

(ii) If the hermitian structure of $\bar{f}$ comes from chosen metrics on $T_{X}, T_{Y}$ and $\omega_{X}, \omega_{Y}$ are equipped with the induced metrics, then we have a commutative diagram

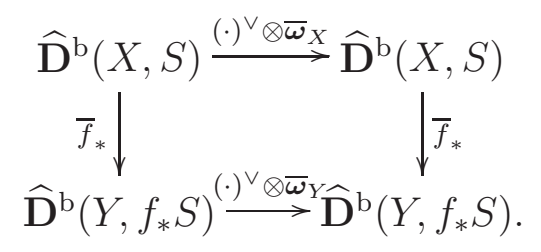

Proof. The first claim is immediate from Theorem 10.9. The second item follows from the first one and the projection formula (Proposition 10.7).

\section{Analytic torsion for degenerating families of curves}

As a second example of application of the theory developed in this article, we describe the singularities of the analytic torsion for degenerating families of curves. The results we prove are particular instances of those obtained by Bismut-Bost [6], Bismut [4] and Yoshikawa [49]. Although the methods of this section can be extended to recover the results of Yoshikawa in [49], for simplicity, we will restrict ourselves to fibrations in curves over a curve.

In fact, our proof is not very different from the one in [4] and [49]. For instance, one of the main ingredients of the proof of the results in [4] and [49] is the Bismut-Lebeau 
immersion formula. Our approach implicitly uses Bismut's generalization of the immersion formula, encoded in the existence of analytic torsion theories for arbitrary projective morphisms. We expect that the techniques of this section can be used to generalize the above results to situations more general than the ones considered by Yoshikawa.

Let $S$ be a smooth complex curve and $f: X \rightarrow S$ a projective morphism of smooth complex varieties, whose fibers are reduced curves with at most ordinary double singular points. We assume that $f$ is generically smooth. Following Bismut-Bost [6, Sec. 2(b)], we call such a family an f.s.o. (famille à singularités ordinaires). The singular locus of $f$, to be denoted $\Sigma$, is a zero dimensional reduced closed subset of $X$. Its direct image $\Delta=f_{*}(\Sigma)$ is the Weil divisor

$$
\Delta=\sum_{p \in S} n_{p} p,
$$

where $n_{p}$ is the number of singular points of the fiber $f^{-1}(p)$. We will abusively identify $\Delta$ with its support. With these notations, we put $V=S \backslash \Delta$. Locally for the analytic topology, the morphism $f$ can be written in complex coordinates either as $f\left(z_{0}, z_{1}\right)=z_{0}$ or $f\left(z_{0}, z_{1}\right)=z_{0} z_{1}\left[6\right.$, Sec. 3(a)]. In the second case, the point of coordinates $\left(z_{0}, z_{1}\right)=$ $(0,0)$ belongs to the singular locus $\Sigma$.

For a vector bundle $F$ over $X$, let $\mathbb{P}(F)$ be the projective space of lines in $F$. The differential $d f: T_{X} \rightarrow f^{*} T_{S}$ induces a section $\mathcal{O}_{X} \rightarrow \Omega_{X} \otimes f^{*} T_{S}$. Because $f$ is smooth over $X \backslash \Sigma$, this section does not vanish on $X \backslash \Sigma$. Therefore there is an induced map

$$
\mu: X \backslash \Sigma \longrightarrow \mathbb{P}\left(\Omega_{X} \otimes f^{*} T_{S}\right) \cong \mathbb{P}\left(\Omega_{X}\right),
$$

called the Gauss map. Notice that this map was already used in [4] and [49].

We next study the blow-up $\widetilde{X}=\mathrm{Bl}_{\Sigma}(X)$ of $X$ at $\Sigma$ and relate it to the Gauss map. Let $\pi: \widetilde{X} \rightarrow X$ be the natural projection. Let $E$ be the exceptional divisor of $\pi$,

$$
E=\bigsqcup_{p \in \Sigma} E_{p}, \quad E_{p} \cong \mathbb{P}\left(T_{p} X\right)
$$

with the reduced scheme structure. For every $p \in \Sigma$, there is an identification $T_{p} X \cong \Omega_{X, p}$ provided by the hessian of $f$, which is a non-degenerate bilinear form on $T_{p} X$. The local description of the blow-up at a point implies:

Lemma 11.1. There is a commutative diagram

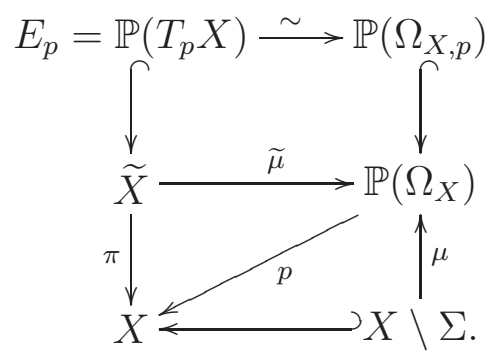

Denote by $\mathcal{O}(-1)$ the tautological divisor either on $\mathbb{P}\left(\Omega_{X}\right)$ or on $E_{p}$. Then there is a natural isomorphism $\left.\widetilde{\mu}^{*} \mathcal{O}(-1)\right|_{E_{p}} \cong \mathcal{O}(-1)$. 
Consider now the short exact sequence of vector bundles on $\mathbb{P}\left(\Omega_{X}\right)$

$$
0 \rightarrow \mathcal{O}(-1) \rightarrow p^{*} \Omega_{X} \rightarrow Q \rightarrow 0
$$

where $Q$ is the universal quotient bundle. Observe that $Q$ is of rank 1 . The dual exact sequence is

$$
0 \rightarrow U \rightarrow p^{*} T_{X} \rightarrow \mathcal{O}(1) \rightarrow 0,
$$

$U$ being the universal vector subsheaf. We denote by $\eta$ the induced exact sequence on $\widetilde{X}$

$$
\eta: 0 \rightarrow \widetilde{\mu}^{*} U \rightarrow \pi^{*} T_{X} \rightarrow \widetilde{\mu}^{*} \mathcal{O}(1) \rightarrow 0
$$

From (11.2) and the definition $\omega_{X / S}=\omega_{X} \otimes f^{*} T_{S}$, we derive a natural isomorphism

$$
\widetilde{\mu}^{*} U \otimes \pi^{*} \omega_{X / S} \cong \widetilde{\mu}^{*} \mathcal{O}(-1) \otimes \tilde{f}^{*} T_{S}
$$

Lemma 11.4. We have

$$
\widetilde{\mu}^{*} \mathcal{O}(-1) \otimes \tilde{f}^{*} T_{S}=\mathcal{O}(E)
$$

Proof. First of all we observe that $\widetilde{\mu}^{*} U \otimes \pi^{*} \omega_{X / S}$ is trivial on the open $W=\widetilde{X} \backslash E$. Indeed, by construction of the Gauss map we have

$$
\left.\tilde{\mu}^{*} U\right|_{W}=\left.\operatorname{ker}\left(d f: T_{X} \rightarrow f^{*} T_{S}\right)\right|_{W}=\omega_{X / S}^{\vee} \mid W .
$$

Hence by equation (11.3) we can write

$$
\tilde{\mu}^{*} \mathcal{O}(-1) \otimes \tilde{f}^{*} T_{S}=\mathcal{O}\left(\sum_{p \in \Sigma} m_{p} E_{p}\right)
$$

To compute the multiplicities $m_{p}$ we use that $\left.\tilde{\mu}^{*} \mathcal{O}(-1)\right|_{E_{p}}=\mathcal{O}(-1),\left(E_{p} \cdot \tilde{f}^{*} T_{S}\right)=0$ and $\left(E_{p} \cdot E_{p}\right)=-1$ :

$$
-m_{p}=\left.\operatorname{deg}\left(\widetilde{\mu}^{*} \mathcal{O}(-1) \otimes \widetilde{f}^{*} T_{S}\right)\right|_{E_{p}}=-1+0=-1
$$

The lemma follows.

Later we will need the commutative diagram of exact sequences

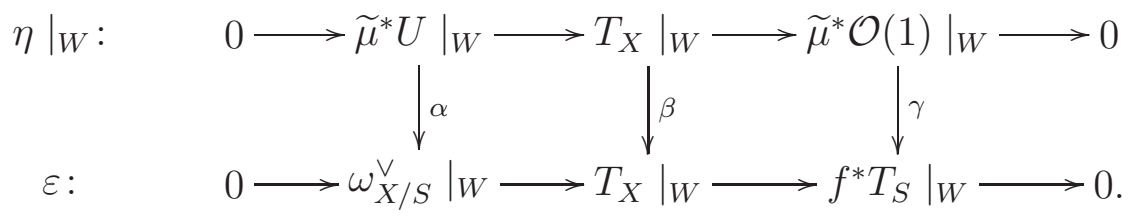

After the identification $\widetilde{\mu}^{*} \mathcal{O}(-1) \otimes \tilde{f}^{*} T_{S}=\mathcal{O}(E)$ provided by the lemma, the morphism $\gamma$ is the restriction to $W$ of the natural inclusion $\widetilde{\mu}^{*} \mathcal{O}(1) \rightarrow \widetilde{\mu}^{*} \mathcal{O}(1) \otimes \mathcal{O}(E)$. This fact will be used below.

We now proceed to introduce the hermitian vector bundles and the analytic torsion classes we aim to study. We fix a theory of generalized analytic torsion classes $T$. 
Let $f: X \rightarrow S, \tilde{f}: \widetilde{X} \rightarrow S$ be f.s.o. as above. Recall that we write $W=X \backslash \Sigma=$ $\widetilde{X} \backslash E$ and $V=S \backslash \Delta$, so that $f^{-1}(V) \subset W$. We endow the tangent spaces $T_{X}$ and $T_{S}$ with smooth hermitian metrics. We will denote by $\bar{f}$ the corresponding morphism in the category $\overline{\mathbf{S m}}_{* / \mathbb{C}}$. On the open subset $W$, there is a quasi-isomorphism

$$
\left.\omega_{X / S}^{\vee}\right|_{W}=\left.\omega_{X / S}^{\vee}[1]\right|_{W} \rightarrow T_{f}
$$

induced by the identification $\left.\omega_{X / S}^{\vee}\right|_{W}=\operatorname{ker}\left(\left.T_{X}\right|_{W} \rightarrow f^{*} T_{S}\right)$. On $\left.\omega_{X / S}^{\vee}\right|_{W}$, and in particular on $\omega_{f^{-1}(V) / V}^{\vee}$, we will put the metric induced by $\left.\overline{T_{X}}\right|_{W}$. We will write $\bar{f}^{\prime}: f^{-1}(V) \rightarrow$ $V$ for the corresponding morphism in $\overline{\mathbf{S m}}_{* / \mathbb{C}}$. Observe that the restriction of $f$ to $W$, and hence to $f^{-1}(V)$, may be identified with the restriction of $\widetilde{f}$. Let $\overline{\mathcal{F}}$ be an object in $\overline{\mathbf{D}}^{\mathrm{b}}(X)$ and fix a hermitian structure on $f_{*} \mathcal{F}$. Then we consider the relative metrized complexes

$$
\bar{\xi}=\left(\bar{f}, \overline{\mathcal{F}}, \overline{f_{*} \mathcal{F}}\right), \quad \bar{\xi}^{\prime}=\left(\bar{f}^{\prime},\left.\overline{\mathcal{F}}\right|_{f^{-1}(V)},\left.\overline{f_{*} \mathcal{F}}\right|_{V}\right),
$$

and the corresponding analytic torsion classes

$$
T(\bar{\xi}) \in \bigoplus_{p} \widetilde{\mathcal{D}}_{D}^{2 p-1}\left(S, N_{f}, p\right), \quad T\left(\bar{\xi}^{\prime}\right) \in \bigoplus_{p} \widetilde{\mathcal{D}}_{D}^{2 p-1}(V, \emptyset, p) .
$$

By the functoriality of analytic torsion classes and the anomaly formulas, we have

$$
T\left(\bar{\xi}^{\prime}\right)=\left.T(\bar{\xi})\right|_{V}-\bar{f}_{b}\left[\operatorname{ch}\left(\left.\overline{\mathcal{F}}\right|_{f^{-1}(V)}\right) \widetilde{\mathrm{Td}}_{m}\left(\left.\bar{\varepsilon}\right|_{f^{-1}(V)}\right)\right] .
$$

Here $\bar{\varepsilon}$ is the exact sequence in (11.6), with the hermitian metrics we have just defined. From now on we will omit the reference to $f^{-1}(V)$ and $V$ in the formulas.

We consider the hermitian structures on the sheaves $U$ and $\mathcal{O}(1)$ on $\mathbb{P}\left(\Omega_{X}\right)$ induced by $p^{*} \overline{T_{X}}$. We will write $\bar{\eta}$ for the exact sequence in (11.2) and $\bar{\alpha}, \bar{\beta}$ and $\bar{\gamma}$ for the vertical isomorphisms in diagram (11.6), all provided with the corresponding metrics. Notice that $\bar{\alpha}$ and $\bar{\beta}$ are isometries. By the properties of the Bott-Chern class $\widetilde{\mathrm{Td}}_{m}$, we have

$$
\widetilde{\mathrm{Td}}_{m}(\bar{\varepsilon})=\widetilde{\mathrm{Td}}_{m}(\bar{\eta})+\operatorname{Td}(\bar{\eta}) \widetilde{\mathrm{Td}}_{m}(\bar{\gamma})
$$

Hence, from (11.7)-(11.8) and identifying $f$ with $\tilde{f}$ over $V$, we have

$$
\begin{aligned}
T\left(\bar{\xi}^{\prime}\right)=T(\bar{\xi})-\widetilde{f}_{*}\left[\pi^{*} \operatorname{ch}(\overline{\mathcal{F}}) \pi^{*} \operatorname{Td}(\bar{f})\right. & \left.\widetilde{T d}_{m}(\bar{\eta})\right] \\
& -\widetilde{f}_{*}\left[\pi^{*} \operatorname{ch}(\overline{\mathcal{F}}) \pi^{*} \operatorname{Td}(\bar{f}) \operatorname{Td}(\bar{\eta}) \widetilde{T d}_{m}(\bar{\gamma})\right] .
\end{aligned}
$$

It will be convenient to have a precise description of $\widetilde{\mathrm{Td}}_{m}(\bar{\gamma})$ at our disposal.

For shorthand, we write $L:=\widetilde{\mu}^{*} \mathcal{O}(1)$ and $\|\cdot\|_{0}$ for its hermitian structure constructed before. We denote by $\|\cdot\|_{1}$ the metric on $\mathcal{O}(E)$ such that the isomorphism $\overline{\mathcal{O}(E)_{1}}=$ $\bar{L}_{0}^{-1} \otimes \tilde{f}^{*} \overline{T_{S}}$ (Lemma 11.4) is an isometry. Recall that $\gamma$ gets identified with the restriction to $W$ of the natural inclusion $L \rightarrow L \otimes \mathcal{O}(E)$. We let $\|\cdot\|_{\infty}$ be the hermitian metric on $\left.L\right|_{W}$ such that $\gamma$ is an isometry. Hence, if 1 denotes the canonical section of $\mathcal{O}(E)$ and $\ell$ is any section of $\left.L\right|_{W}$, then we have

$$
\|\ell\|_{\infty}=\|\ell\|_{0}\|\mathbf{1}\|_{1}
$$


To simplify the notations, we will skip the reference to $W$. We then have on $W$

$$
\widetilde{\mathrm{Td}}_{m}(\bar{\gamma})=\widetilde{\mathrm{Td}}_{m}\left(\bar{L}_{0} \stackrel{\text { id }}{\rightarrow} \bar{L}_{\infty}\right)
$$

To compute a representative of this class, we fix a smooth function $h: \mathbb{P}_{\mathbb{C}}^{1} \rightarrow \mathbb{R}$ such that $h(0)=0$ and $h(\infty)=1$. Then we proceed by a deformation argument. Let $q: W \times \mathbb{P}_{\mathbb{C}}^{1} \rightarrow$ $W$ be the projection to the first factor. On the line bundle $q^{*} L$ we put the metric that, on the fiber at the point $(w, t) \in W \times \mathbb{P}_{\mathbb{C}}^{1}$, is determined by the formula

$$
\|\ell\|_{(w, t)}=\|\ell\|_{0, w}\|\mathbf{1}\|_{1, w}^{h(t)} .
$$

We will write $\|\cdot\|_{t}$ for this family of metrics parametrized by $\mathbb{P}_{\mathbb{C}}^{1}$. Define

$$
\overline{\operatorname{Td}}\left(\bar{L}_{0} \rightarrow \bar{L}_{\infty}\right)=\frac{1}{2 \pi i} \int_{\mathbb{P}_{\mathbb{C}}^{1}} \frac{-1}{2} \log (t \bar{t})\left(\operatorname{Td}\left({\overline{q^{*}}}_{t}\right)-\operatorname{Td}\left({\overline{q^{*} L_{0}}}\right)\right)
$$

Then

$$
\overline{\operatorname{Td}}_{m}(\bar{\gamma})=\operatorname{Td}^{-1}\left(\bar{L}_{0}\right) \overline{\operatorname{Td}}\left(\bar{L}_{0} \rightarrow \bar{L}_{\infty}\right)
$$

represents the class $\widetilde{\mathrm{Td}}_{m}(\gamma)$. Let us develop $\overline{\mathrm{Td}}_{m}(\bar{\gamma})$. If $\overline{\mathcal{O}}_{t}$ denotes the trivial line bundle on $W \times \mathbb{P}_{\mathbb{C}}^{1}$ with the norm $\|\mathbf{1}\|_{t}=\|\mathbf{1}\|_{1}^{h(t)}$, then we compute

$$
\operatorname{Td}\left({\overline{q^{*}}}_{t}\right)-\operatorname{Td}\left({\overline{q^{*}}}_{0}\right)=\frac{1}{2} c_{1}\left(\overline{\mathcal{O}}_{t}\right)+\frac{1}{6} c_{1}\left(\overline{\mathcal{O}}_{t}\right) q^{*} c_{1}\left(\bar{L}_{0}\right)+\frac{1}{12} c_{1}\left(\overline{\mathcal{O}}_{t}\right)^{2} .
$$

By the very definition of $c_{1}$, we find

$$
\begin{aligned}
& c_{1}\left(\overline{\mathcal{O}}_{t}\right)=\partial \bar{\partial} \log \|\mathbf{1}\|_{t}^{2}=\partial \bar{\partial}\left(h(t) \log \|\mathbf{1}\|_{1}^{2}\right) \\
& \quad=h(t) c_{1}\left({\overline{\mathcal{O}(E)_{1}}}_{1}\right)+\log \|\mathbf{1}\|_{1}^{2} \partial \bar{\partial} h(t)+\partial h(t) \wedge \bar{\partial} \log \|\mathbf{1}\|_{1}^{2}+\partial \log \|\mathbf{1}\|_{1}^{2} \wedge \bar{\partial} h(t) .
\end{aligned}
$$

We easily obtain

$$
\begin{aligned}
& \frac{1}{2 \pi i} \int_{\mathbb{P}_{\mathbb{C}}^{1}} \frac{-1}{2} \log (t \bar{t}) \frac{1}{2} c_{1}\left(\overline{\mathcal{O}}_{t}\right)=-\frac{1}{2} \log \|\mathbf{1}\|_{1}, \\
& \frac{1}{2 \pi i} \int_{\mathbb{P}_{\mathbb{C}}^{1}} \frac{-1}{2} \log (t \bar{t}) \frac{1}{6} q^{*} c_{1}\left(\bar{L}_{0}\right) c_{1}\left(\overline{\mathcal{O}}_{t}\right)=-\frac{1}{6} \log \|\mathbf{1}\|_{1} c_{1}\left(\bar{L}_{0}\right) .
\end{aligned}
$$

With some more work, we have

$$
\begin{aligned}
\frac{1}{2 \pi i} \int_{\mathbb{P}_{\mathbb{C}}^{1}} \frac{-1}{2} \log (t \bar{t}) \frac{1}{12} c_{1}\left(\overline{\mathcal{O}}_{t}\right)^{2}= & -\frac{a}{6} \log \|\mathbf{1}\|_{1} c_{1}\left(\overline{\mathcal{O}(E)_{1}}\right) \\
& +\frac{b}{3} \partial\left(\log \|\mathbf{1}\|_{1} \bar{\partial} \log \|\mathbf{1}\|_{1}\right),
\end{aligned}
$$

where

$$
a=\frac{1}{2 \pi i} \int_{\mathbb{P}_{\mathbb{C}}^{1}} \log (t \bar{t}) \frac{1}{2} \partial \bar{\partial}\left(h(t)^{2}\right), \quad b=\frac{1}{2 \pi i} \int_{\mathbb{P}_{\mathbb{C}}^{1}} \log (t \bar{t}) \partial h(t) \wedge \bar{\partial} h(t) .
$$


We observe that

$$
a=\frac{1}{2 \pi i} \int_{\mathbb{P}_{\mathbb{C}}^{1}} \log (t \bar{t}) \frac{1}{2} \partial \bar{\partial}\left(h(t)^{2}\right)=\frac{1}{2},
$$

which is independent of $h$. All in all, equations 11.10-11.14 provide the following expression for the representative $\overline{\mathrm{Td}}_{m}(\bar{\gamma})$ of $\widetilde{\mathrm{Td}}_{m}(\bar{\gamma})$ :

$$
\begin{aligned}
\overline{\mathrm{Td}}_{m}(\bar{\gamma})=\operatorname{Td}^{-1}\left(\bar{L}_{0}\right)( & -\frac{1}{2} \log \|\mathbf{1}\|_{1}-\frac{1}{6} \log \|\mathbf{1}\|_{1} c_{1}\left(\bar{L}_{0}\right) \\
& \left.-\frac{1}{12} \log \|\mathbf{1}\|_{1} c_{1}\left(\overline{\mathcal{O}(E)_{1}}\right)+\frac{b}{3} \partial\left(\log \|\mathbf{1}\|_{1} \bar{\partial} \log \|\mathbf{1}\|_{1}\right)\right) .
\end{aligned}
$$

Given a current $\eta \in \mathcal{D}_{D}^{n}(X, p)$, we will call $(n, p)$ its Deligne bidegree, while we will call the Dolbeault bidegree to the bidegree in the Dolbeault complex. When it is clear from the context to which bidegree we are referring, we call it bidegree.

We now study the singularities of the component of Deligne bidegree $(1,1)$ of $T\left(\bar{\xi}^{\prime}\right)$ near the divisor $\Delta$. For this we first recall the decomposition of equation (11.9). Observe that $\widetilde{\mathcal{D}}_{D}^{1}(V, \emptyset, 1)$ gets identified with the space of smooth real functions on $V$. In the sequel, for an element $\vartheta \in \oplus_{p} \widetilde{D}_{D}^{2 p-1}(*, p)$, we write $\vartheta^{(2 r-1, r)}$ to refer to its component of bidegree $(2 r-1, r)$. By construction of the Deligne complex, an element of Deligne bidegree $(2 r-1, r)$ is just a current of Dolbeault bidegree $(r-1, r-1)$.

The following assertion is well-known. See for instance [47, Lemma 2.1, Cor. 2.2].

Lemma 11.16. Let $\Omega \subset \mathbb{C}$ be an open subset and $\vartheta$ a current of Dolbeault bidegree $(0,0)$ on $\Omega$. Let $\Delta$ be the standard laplacian. If the current $\Delta \vartheta$ is represented by a locally bounded measurable function, then $\vartheta$ is represented by a continuous function.

Proposition 11.17. The current $T(\bar{\xi})^{(1,1)} \in \widetilde{\mathcal{D}}_{D}^{1}\left(S, N_{f}, 1\right)$ is represented by a continuous function on $S$.

Proof. The differential equation satisfied by $T(\bar{\xi})^{(1,1)}$ is

$$
\mathrm{d}_{\mathcal{D}} T(\bar{\xi})^{(1,1)}=\operatorname{ch}\left(\overline{f_{*} \mathcal{F}}\right)^{(2,1)}-f_{*}[\operatorname{ch}(\overline{\mathcal{F}}) \operatorname{Td}(\bar{f})]^{(2,1)} .
$$

In local coordinates, the operator $\mathrm{d}_{\mathcal{D}}=-2 \partial \bar{\partial}$ is a rescaling of the laplacian $\Delta$. By the lemma, it is enough we prove that the current at the right hand side of 11.18 is represented by a locally bounded measurable differential form. Because $\operatorname{ch}\left(\overline{f_{*} \mathcal{F}}\right)^{(2,1)}$ and $\operatorname{ch}(\overline{\mathcal{F}}) \operatorname{Td}(\bar{f})$ are smooth differential forms, we are reduced to study currents of the form $f_{*}[\theta]^{(2,1)}$, where $\theta$ is a smooth differential form. By a partition of unity argument, we reduce to the case where $f: \mathbb{C}^{2} \rightarrow \mathbb{C}$ is the morphism $f\left(z_{0}, z_{1}\right)=z_{0} z_{1}$ and $\theta$ is a differential form of Dolbeault bidegree $(2,2)$ with compact support. Then we need to prove that the fiber integral

$$
G(w)=\int_{z_{0} z_{1}=w} \theta
$$

is a bounded form in a neighborhood of $w=0$. Write $\theta=h\left(z_{0}, z_{1}\right) d z_{0} \wedge d \bar{z}_{0} \wedge d z_{1} \wedge d \bar{z}_{1}$. We reduce to study integrals of the form

$$
G(w)=\left(\int_{|w|<\left|z_{0}\right|<1} h\left(z_{0}, z_{0} / w\right) \frac{|w|^{2}}{\left|z_{0}\right|^{4}} d z_{0} \wedge \bar{z}_{0}\right) d w \wedge d \bar{w} .
$$

The property follows from an easy computation in polar coordinates. 
Proposition 11.19. Let $\theta$ be a differential form of Dolbeault bidegree $(1,1)$ on $\tilde{X}$. Then the current $\tilde{f}_{*}[\theta]$ is represented by a bounded function on $S$.

Proof. The proof is the same as in [6, Prop. 5.2]. One only has to show that the argument in loc. cit. carries over to the case of the non-reduced fibres that have appeared when blowing up the nodes.

Corollary 11.20. The current $\widetilde{f}_{*}\left[\pi^{*} \operatorname{ch}(\overline{\mathcal{F}}) \pi^{*} \operatorname{Td}(\bar{f}) \widetilde{T d}_{m}(\bar{\eta})\right]$ is represented by a bounded function on $S$.

Proof. It suffices to observe that the differential form $\pi^{*} \operatorname{ch}(\overline{\mathcal{F}}) \pi^{*} \operatorname{Td}(\bar{f}) \widetilde{\mathrm{Td}}_{m}(\bar{\eta})$ is actually smooth on the whole $\tilde{X}$.

According to 11.9), it remains to study the current

$$
\left.\widetilde{f}_{*}\left[\pi^{*} \operatorname{ch}(\overline{\mathcal{F}}) \pi^{*} \operatorname{Td}(\bar{f}) \operatorname{Td}(\bar{\eta}) \widetilde{\operatorname{Td}}_{m}(\bar{\gamma})\right]\right|_{V}
$$

The main difference with the situation in Corollary 11.20 is that the class $\widetilde{\mathrm{Td}}_{m}(\bar{\gamma})$ is not defined on the whole $\widetilde{X}$, but only on $W=\widetilde{X} \backslash E$. In the following discussion we will use the representative $\overline{\mathrm{Td}}_{m}(\bar{\gamma})$ defined in (11.10) at the place of $\widetilde{\mathrm{Td}}_{m}(\bar{\gamma})$. In view of equations (11.11)-11.13), the first result we need is the following statement.

Proposition 11.21. Let $\theta$ be a smooth and $\partial, \bar{\partial}$ closed differential form on $\widetilde{X}$, of Dolbeault bidegree $(1,1)$. Let $w$ be an analytic coordinate in a neighborhood of $p \in \Delta$ with $w(p)=$ 0 . Write $D_{p}=E \cap \widetilde{f}^{-1}(p)$. Then, the current

$$
\widetilde{f}_{*}\left[\log \|\mathbf{1}\|_{1} \theta\right]-\left(\frac{1}{2 \pi i} \int_{D_{p}} \theta\right)[\log |w|]
$$

is represented by a continuous function in a neighborhood of $p$. In particular, if $\theta$ is cohomologous to a form $\pi^{*} \vartheta$, where $\vartheta$ is a smooth and $\partial, \bar{\partial}$ closed differential form on $X$, then $\widetilde{f}_{*}\left[\log \|\mathbf{1}\|_{1} \theta\right]$ is represented by a continuous function on $S$.

Proof. Recall that the Poincaré-Lelong formula provides the equality of currents

$$
\mathrm{d}_{\mathcal{D}}\left[\log \|\mathbf{1}\|_{1}^{-1}\right]=\left[c_{1}\left(\overline{\mathcal{O}(E)_{1}}\right)\right]-\delta_{E} .
$$

Moreover, the operator $\mathrm{d}_{\mathcal{D}}$ commutes with proper push-forward. Therefore, taking into account that $\theta$ is $\partial$ and $\bar{\partial}$ closed, the equation

$$
\mathrm{d}_{\mathcal{D}} \widetilde{f}_{*}\left[\log \|\mathbf{1}\|_{1} \theta\right]=\left(\frac{1}{2 \pi i} \int_{D_{p}} \theta\right) \delta_{p}-\widetilde{f}_{*}\left[c_{1}\left(\overline{\mathcal{O}(E)_{1}}\right) \theta\right] .
$$

holds in a neighborhood of $p$. On the other hand, the Poincaré-Lelong equation also gives $\mathrm{d}_{\mathcal{D}}[\log |w|]=\delta_{p}$. Using (11.22), we see that

$$
\mathrm{d}_{\mathcal{D}}\left(\widetilde{f}_{*}\left[\log \|\mathbf{1}\|_{1} \theta\right]-\left(\frac{1}{2 \pi i} \int_{D_{p}} \theta\right)[\log |w|]\right)=-\frac{1}{2} \widetilde{f}_{*}\left[c_{1}(\overline{\mathcal{O}(E)}) \theta\right] .
$$


Finally, by Proposition 11.19 , the current $\widetilde{f}_{*}\left[c_{1}(\overline{\mathcal{O}(E)}) \theta\right]$ is represented by a continuous function on $S$. Hence the first assertion follows from Lemma 11.16, For the second assertion, we just observe that, in this case,

$$
\int_{D_{p}} \theta=\int_{D_{p}} \pi^{*} \vartheta=0
$$

The proof is complete.

Corollary 11.23. Let $n_{p}$ be the multiplicity of $\Delta$ at $p$ and $O(1)$ the current represented by a locally bounded function. The following estimates hold in a neighborhood of $p$

$$
\begin{aligned}
& \widetilde{f}_{*}\left[\log \|\mathbf{1}\|_{1} c_{1}\left(\pi^{*} \overline{T_{X}}\right)\right]=O(1), \\
& \widetilde{f}_{*}\left[\log \|\mathbf{1}\|_{1} c_{1}\left(\overline{\mathcal{O}(E)_{1}}\right)\right]=-n_{p}[\log |w|]+O(1), \\
& \widetilde{f}_{*}\left[\log \|\mathbf{1}\|_{1} c_{1}\left(\bar{L}_{0}\right)\right]=n_{p}[\log |w|]+O(1), \\
& \widetilde{f}_{*}\left[\log \|\mathbf{1}\|_{1} c_{1}\left(\widetilde{\mu}^{*} \bar{U}\right)\right]=-n_{p}[\log |w|]+O(1) .
\end{aligned}
$$

Proof. We use (11.3)-(11.5) and the intersection numbers $\left(D_{p} \cdot D_{p}\right)=\left(D_{p} \cdot E\right)=-n_{p}$.

Corollary 11.24. With the notations above, the development

$$
\widetilde{f}_{*}\left[\pi^{*} \operatorname{ch}(\overline{\mathcal{F}}) \pi^{*} \operatorname{Td}(\bar{f}) \operatorname{Td}(\bar{\eta}) \widetilde{\operatorname{Td}}_{m}(\bar{\gamma})\right]^{(3,2)}=\operatorname{rk}(\overline{\mathcal{F}}) \frac{n_{p}}{6}[\log |w|]+O(1)
$$

holds in a neighborhood of $p$.

Proof. We take into account the expression (11.15) for the representative $\overline{\mathrm{Td}}_{m}(\bar{\gamma})$, the developments of the smooth differential forms $\operatorname{ch}(\overline{\mathcal{F}}), \operatorname{Td}(\bar{f}), \operatorname{Td}(\bar{\eta})$ and $\operatorname{Td}^{-1}\left(\bar{L}_{0}\right)$, and then apply Corollary 11.23 . We find

$$
\begin{aligned}
\tilde{f}_{*}\left[\pi^{*} \operatorname{ch}(\overline{\mathcal{F}}) \pi^{*} \operatorname{Td}(\bar{f})\right. & \left.\operatorname{Td}(\bar{\eta}) \widetilde{\operatorname{Td}}_{m}(\bar{\gamma})\right]^{(3,2)}= \\
& \operatorname{rk}(\overline{\mathcal{F}}) \frac{n_{p}}{6}[\log |w|]+\operatorname{rk}(\overline{\mathcal{F}}) \frac{b}{3} \widetilde{f}_{*}\left[\partial\left(\log \|\mathbf{1}\|_{1} \bar{\partial} \log \|\mathbf{1}\|_{1}\right)\right]+O(1) .
\end{aligned}
$$

To conclude we observe that, on $V$, the term $\widetilde{f}_{*}\left[\partial\left(\log \|\mathbf{1}\|_{1} \bar{\partial} \log \|\mathbf{1}\|_{1}\right)\right]$ vanishes. Indeed, the morphism $\tilde{f}_{*}$ is smooth on $V$ with one dimensional fibers. Hence this current is represented by the function

$$
V \ni s \mapsto \frac{1}{2 \pi i} \int_{\widetilde{f}^{-1}(s)} \partial\left(\log \|\mathbf{1}\|_{1} \bar{\partial} \log \|\mathbf{1}\|_{1}\right)=\frac{1}{2 \pi i} \int_{\widetilde{f}^{-1}(s)} d\left(\log \|\mathbf{1}\|_{1} \bar{\partial} \log \|\mathbf{1}\|_{1}\right)=0 .
$$

This ends the proof.

The results of this section are summarized in the following statement.

Theorem 11.25. Let $p \in \Delta$ and let $n_{p}$ be the number of singular points of $f: X \rightarrow S$ lying above $p$. Let $w$ be a local coordinate on $S$, centered at $p$. Then, in a neighborhood of $p$, we have the estimate

$$
T\left(\bar{\xi}^{\prime}\right)^{(1,1)}=-\frac{\mathrm{rk} \overline{\mathcal{F}}}{6} n_{p}[\log |w|]+O(1) .
$$


Proof. It is enough to join (11.9), Proposition 11.17, Corollary 11.20 and 11.24.

Corollary 11.26. Assume that $\overline{\mathcal{F}}=\bar{E}$ is a vector bundle placed in degree 0 , and that $R^{1} f_{*} E=0$ on $S$. Endow $f_{*} E$ with the $L^{2}$ metric on $V$ depending on $\bar{E}$ and the metric on $\overline{\omega_{f^{-1}(V) / V}}$. Write $\xi^{\prime \prime}=\left(\bar{f}^{\prime}, \bar{E}, \bar{f}_{*} E_{L^{2}}\right)$ for the corresponding relative metrized complex on $V$. Let $p$ and $w$ be as in the theorem. Then we have

$$
T\left(\bar{\xi}^{\prime \prime}\right)^{(1,1)}=-\frac{\operatorname{rk}(\overline{\mathcal{F}})}{6} n_{p}[\log |w|]+O\left(\log \log |w|^{-1}\right)
$$

as $w \rightarrow 0$.

Proof. Introduce an auxiliary smooth hermitian metric on the vector bundle $f_{*} E$ on $S$, and let $\bar{\xi}^{\prime}=\left(\bar{f}^{\prime}, \bar{E}, \overline{f_{*} E}\right)$ be the corresponding relative metrized complex. Then the theorem applies to $\bar{\xi}^{\prime}$. By the anomaly formulas, on $V$ we have

$$
T\left(\bar{\xi}^{\prime \prime}\right)^{(1,1)}=T\left(\bar{\xi}^{\prime}\right)^{(1,1)}+\widetilde{\operatorname{ch}}\left(\overline{f_{*} E}, \bar{f}_{*} E_{L^{2}}\right)^{(1,1)} .
$$

By [6, Prop. 7.1], the $L^{2}$ metric has logarithmic singularities near $w=0$ and

$$
\widetilde{\operatorname{ch}}\left(\bar{f}_{*} E, \bar{f}_{*} E_{L^{2}}\right)=O\left(\log \log |w|^{-1}\right)
$$

as $w \rightarrow 0$. This proves the corollary.

Remark 11.27. The corollary is to be compared with [6, Thm. 9.3]. The difference of sign is due to the fact that Bismut and Bost work with the inverse of the usual determinant line bundle. The approach of loc. cit. is more analytic and requires the spectral description of the Ray-Singer analytic torsion.

Acknowledgments. During the elaboration of this paper we have benefited from conversations with many colleagues, that helped us to understand some points, to clarify others or to find the relevant bibliography. Our thanks to J.-M. Bismut, J.-B. Bost, D. Burghelea, D. Eriksson, J. Kramer, U. Kühn, X. Ma, V. Maillot, D. Rössler, C. Soulé.

We would like to thank the following institutions where part of the research conducting to this paper was done: the CRM in Bellaterra (Spain), the CIRM in Luminy (France), the Morningside Institute of Beijing (China), the University of Barcelona and the IMUB, the Alexandru Ioan Cuza University of Iasi, the Institut de Mathématiques de Jussieu and the ICMAT (Madrid).

Burgos and Freixas were partially supported by grant MTM2009-14163- C02-01, Burgos was partially supported by CSIC research project 2009501001, Liţcanu was partially supported by CNCSIS -UEFISCSU, project number PNII - IDEI 2228/2008.

\section{References}

[1] A. Berthomieu and J.-M. Bismut, Quillen metrics and higher analytic torsion forms, J. Reine Angew. Math. 457 (1994), 85-184.

[2] J.-M. Bismut, The Atiyah-Singer index theorem for families of Dirac operators: two heat equation proofs, Invent. Math. 83 (1985), no. 1, 91-151.

[3] Holomorphic families of immersions and higher analytic torsion forms, Astérisque, vol. 244, SMF, 1997. 
[4] Quillen metrics and singular fibers in arbitrary relative dimension, J. Alg. Geom. 6 (1997), $19-149$.

[5] _ Holomorphic and de Rham torsion, Comp. Math. 140 (2004), 1302-1356.

[6] J.-M. Bismut and J.-B. Bost, Fibrés déterminants, métriques de Quillen et dégénérescence des courbes, Acta Math. 165 (1990), 1-103.

[7] J. M. Bismut and D. S. Freed, The analysis of elliptic families. I. Metrics and connections on determinant bundles, Comm. Math. Phys. 106 (1986), no. 1, 159-176.

[8] The analysis of elliptic families. II. Dirac operators, eta invariants, and the holonomy theorem, Comm. Math. Phys. 107 (1986), no. 1, 103-163.

[9] J.-M. Bismut, H. Gillet, and C. Soulé, Analytic torsion and holomorphic determinant bundles I, Comm. Math. Phys. 115 (1988), 49-78.

[10] _ Analytic torsion and holomorphic determinant bundles II, Comm. Math. Phys. 115 (1988), $79-126$.

[11] Analytic torsion and holomorphic determinant bundles III, Comm. Math. Phys. 115 (1988), 301-351.

[12] Complex immersions and Arakelov geometry, The Grothendieck Festschrift, Vol. I, Progr. Math., vol. 86, Birkhäuser Boston, Boston, MA, 1990, pp. 249-331.

[13] J.-M. Bismut and K. Köhler, Higher analytic torsion forms for direct images and anomaly formulas, J. Alg. Geom. 1 (1992), 647-684.

[14] J.-M. Bismut and G. Lebeau, Complex immersions and Quillen metrics, Publ. Math. IHES 74 (1991), $1-298$.

[15] J.-B. Bost, Analytic torsion of projective spaces and compatibility with immersions of Quillen metrics, IMRN 8 (1998), 427-435.

[16] J. I. Burgos Gil, Arithmetic Chow rings and Deligne-Beilinson cohomology, J. Alg. Geom. 6 (1997), $335-377$.

[17] J. I. Burgos Gil, G. Freixas i Montplet, and R. Liţcanu, Hermitian structures on the derived category of coherent sheaves, arXiv:1102.2063.

[18] J. I. Burgos Gil, J. Kramer, and U. Kühn, Cohomological arithmetic Chow rings, J. Inst. Math. Jussieu 6 (2007), no. 1, 1-172.

[19] J. I. Burgos Gil and R. Liţcanu, Singular Bott-Chern classes and the arithmetic GrothendieckRiemann-Roch theorem for closed immersions, Doc. Math. 15 (2010), 73-176.

[20] P. Deligne, Le déterminant de la cohomologie, Current Trends in Arithmetic Algebraic Geometry (K.A. Ribet, ed.), Contemporary Mathematics, vol. 67, AMS, 1985, pp. 93-177.

[21] P Deligne and D. Mumford, The irreducibility of the space of curves of given genus, Publ. Math. IHES 36 (1969), 75-109.

[22] C. Faber and R. Pandharipande, Logarithmic series and Hodge integrals in the tautological ring. with an appendix by Don Zagier., Michigan Math. J. 48 (2000), 215-252.

[23] G. Faltings, Calculus on arithmetic surfaces, Annals of Math. 119 (1984), 387-424.

[24] Lectures on the arithmetic Riemann-Roch theorem, Annals of Math. Studies, vol. 127, Princeton University Press, 1992.

[25] H. Gillet, D. Rössler, and C Soulé, An arithmetic Riemann-Roch theorem in higher degrees, Ann. Inst. Fourier 58 (2008), no. 6, 2169-2189.

[26] H. Gillet and C. Soulé, Direct images of Hermitian holomorphic bundles, Bull. Amer. Math. Soc. (N.S.) 15 (1986), no. 2, 209-212. 
[27] Arithmetic intersection theory, Publ. Math. IHES 72 (1990), 94-174.

[28] Characteristic classes for algebraic vector bundles with hermitian metric I, II, Annals of Math. 131 (1990), 163-203,205-238.

[29] __ Analytic torsion and the arithmetic Todd genus, Topology 30 (1991), no. 1, 21-54, With an appendix by D. Zagier.

[30] __ An arithmetic Riemann-Roch theorem, Invent. Math. 110 (1992), 473-543.

[31] A. Grothendieck, Elements de Geometrie Algebrique IV. Quatrième partie, Publ. Math. I.H.E.S. 32 (1967), 5-361.

[32] D. Huybrechts, Fourier-Mukai transforms in algebraic geometry, Oxford mathematical monographs, Oxford University Press, 2006.

[33] K. Igusa, Axioms for higher torsion invariants of smooth bundles, J. Topol. 1 (2008), no. 1, 159-186.

[34] U. Kühn, Generalized arithmetic intersection numbers, J. Reine Angew. Math. 534 (2001), 209-236.

[35] X. Ma, Formes de torsion analytique et familles de submersions. I, Bull. Soc. Math. France 127 (1999), no. 4, 541-621.

[36] _ Formes de torsion analytique et familles de submersions. II, Asian J. Math. 4 (2000), no. 3, 633-667.

[37] V. Maillot and D. Roessler, On the periods of motives with complex multiplication and a conjecture of Gross-Deligne, Ann. of Math. (2) 160 (2004), no. 2, 727-754.

[38] D. Mumford, Lectures on curves on an algebraic surface, Princeton University Press, 1966.

[39] D. Quillen, Higher algebraic K-theory I, Algebraic K-theory I (H. Bass, ed.), Lecture Notes in Math., vol. 341, Springer-Verlag, 1973, pp. 85-147.

[40] _ Determinants of Cauchy-Riemann operators over a Riemann surface, Funct. Anal. Appl. 19 (1985), 31-34.

[41] D. B. Ray and I. M. Singer, R-torsion and the Laplacian on Riemannian manifolds, Advances in Math. 7 (1971), 145-210.

[42] _ Analytic torsion for complex manifolds, Ann. of Math. (2) 98 (1973), 154-177.

[43] D. Roessler, An Adams-Riemann-Roch theorem in Arakelov geometry, Duke Math. J. 96 (1999), 61126.

[44] C. Soulé, D. Abramovich, J.-F. Burnol, and J. Kramer, Lectures on Arakelov Geometry, Cambridge Studies in Advanced Math., vol. 33, Cambridge University Press, 1992.

[45] S. Tang, Uniqueness of equivariant singular Bott-Chern classes, arXiv:1005.5025v1 [math.AG], 2010.

[46] L. Weng, Relative Bott-Chern secondary characteristic classes, arXiv:math/9810117v, 1998.

[47] S. A. Wolpert, On obtaining a positive line bundle from the Weil-Petersson class, Am. J. Math. 107 (1985), 1485-1507.

[48] Chern forms and the riemann tensor for the moduli space of curves, Invent. math. 85 (1986), 119-145.

[49] K.-I. Yoshikawa, On the singularity of Quillen metrics, Math. Ann. 337 (2007), 61-89.

[50] Y. Zha, A general arithmetic Riemann-Roch theorem, Ph.D. thesis, University of Chicago, 1998. 